\title{
British Thoracic Society guidelines for home oxygen use in adults
}

\author{
Maxine Hardinge, ${ }^{1}$ Joe Annandale, ${ }^{2}$ Simon Bourne, ${ }^{3}$ Brendan Cooper, ${ }^{4}$ Angela Evans, ${ }_{1}^{5}$ \\ Daryl Freeman, ${ }^{6}$ Angela Green ${ }^{7}$ Sabrine Hippolyte, ${ }^{8}$ Vikki Knowles, ${ }^{9}$ William MacNee, ${ }^{10}$ \\ Lynn McDonnell, ${ }^{11}$ Kathy Pye, ${ }^{12}$ Jay Suntharalingam, ${ }^{13}$ Vandana Vora, ${ }^{14}$ Tom Wilkinson, ${ }^{15}$ \\ British Thoracic Society Home Oxygen Guideline Development Group, on behalf of the \\ British Thoracic Society Standards of Care Committee
}

- Additional material is published online only. To view please visit the journal online (http://dx.doi.org/10.1136/ thoraxjnl-2015-206865).

For numbered affiliations see end of article.

\section{Correspondence to}

Dr Maxine Hardinge, Oxford Centre of Respiratory Medicine, Churchill Hospital, Oxford University Hospitals NHS Trust, Headington, Oxford OX3 7LJ, UK; maxine.hardinge@ouh. nhs.uk

Received 28 January 2015 Accepted 29 January 2015
CrossMark

$$
\begin{aligned}
& \hline \text { To cite: Hardinge M, } \\
& \text { Annandale J, Bourne S, } \\
& \text { et al. Thorax 2015;70: } \\
& \text { i1-i43. }
\end{aligned}
$$

\section{ABSTRACT}

The British Thoracic Society (BTS) Home Oxygen Guideline provides detailed evidence-based guidance for the use of home oxygen for patients out of hospital. Although the majority of evidence comes from the use of oxygen in patients with chronic obstructive pulmonary disease, the scope of the guidance includes patients with a variety of long-term respiratory illnesses and other groups in whom oxygen is currently ordered, such as those with cardiac failure, cancer and end-stage cardiorespiratory disease, terminal illness or cluster headache. It explores the evidence base for the use of different modalities of oxygen therapy and patientrelated outcomes such as mortality, symptoms and quality of life. The guideline also makes recommendations for assessment and follow-up protocols, and risk assessments, particularly in the clinically challenging area of home oxygen users who smoke. The guideline development group is aware of the potential for confusion sometimes caused by the current nomenclature for different types of home oxygen, and rather than renaming them, has adopted the approach of clarifying those definitions, and in particular emphasising what is meant by long-term oxygen therapy and palliative oxygen therapy. The home oxygen guideline provides expert consensus opinion in areas where clinical evidence is lacking, and seeks to deliver improved prescribing practice, leading to improved compliance and improved patient outcomes, with consequent increased value to the health service.

\section{SUMMARY OF RECOMMENDATIONS AND GOOD PRACTICE POINTS}

Evidence for use of long-term oxygen therapy in patients with chronic obstructive pulmonary disease

- Patients with stable chronic obstructive pulmonary disease (COPD) and a resting $\mathrm{PaO}_{2} \leq 7.3 \mathrm{kPa}$ should be assessed for long-term oxygen therapy (LTOT) which offers survival benefit and improves pulmonary haemodynamics. (Grade A)

- LTOT should be ordered for patients with stable COPD with a resting $\mathrm{PaO}_{2} \leq 8 \mathrm{kPa}$ with evidence of peripheral oedema, polycythaemia (haematocrit $\geq 55 \%$ ) or pulmonary hypertension. (Grade A)

- LTOT should be ordered for patients with resting hypercapnia if they fulfil all other criteria for LTOT. (Grade B)
Evidence for use of LTOT in other respiratory or cardiac disease

- LTOT should be ordered for patients with interstitial lung disease (ILD) with a resting $\mathrm{PaO}_{2}$ $\leq 7.3 \mathrm{kPa}$. (Grade D)

- LTOT should be ordered for patients with ILD with a resting $\mathrm{PaO}_{2} \leq 8 \mathrm{kPa}$ in the presence of peripheral oedema, polycythaemia (haematocrit $\geq 55 \%$ ) or evidence of pulmonary hypertension. (Grade D)

\section{Good practice point}

- Patients with ILD who experience severe breathlessness could be considered for palliative oxygen therapy (POT). $(\sqrt{ })$

LTOT in patients with cystic fibrosis

- LTOT should be ordered for patients with cystic fibrosis (CF) with a resting $\mathrm{PaO}_{2} \leq 7.3 \mathrm{kPa}$. (Grade D)

- LTOT should be ordered for patients with CF with a resting $\mathrm{PaO}_{2} \leq 8 \mathrm{kPa}$ in the presence of peripheral oedema, polycythaemia (haematocrit $\geq 55 \%$ ) or evidence of pulmonary hypertension. (Grade D)

LTOT in patients with pulmonary hypertension

- LTOT should be ordered for patients with pulmonary hypertension, including idiopathic pulmonary hypertension, when the $\mathrm{PaO}_{2}$ is $\leq 8 \mathrm{kPa}$. (Grade D)

LTOT in patients with neuromuscular or chest wall disorders

- Non-invasive ventilation (NIV) should be the treatment of choice for patients with chest wall or neuromuscular disease causing type 2 respiratory failure. Additional LTOT may be required in case of hypoxaemia not corrected with NIV. (Grade D)

LTOT in patients with advanced cardiac failure

- LTOT should be ordered for patients with advanced cardiac failure with a resting $\mathrm{PaO}_{2}$ $\leq 7.3 \mathrm{kPa}$. (Grade D)

- LTOT should be ordered for patients with advanced cardiac failure with a resting $\mathrm{PaO}_{2}$ $\leq 8 \mathrm{kPa}$ in the presence of peripheral oedema, polycythaemia (haematocrit $\geq 55 \%$ ) or evidence of pulmonary hypertension on ECG or echocardiograph. (Grade D) 
Outcomes of LTOT in patients who continue to smoke

- If LTOT is ordered for patients who are continuing to smoke, the potential for more limited clinical benefit should be discussed with the patient. (Grade D)

Referral and assessment of patients for LTOT

- Written and verbal information should be given to patients referred to home oxygen assessment services at the time of referral. (Grade D)

- Patients with a resting stable oxygen saturation $\left(\mathrm{SpO}_{2}\right)$ of $\leq 92 \%$ should be referred for a blood gas assessment in order to assess eligibility for LTOT. (Grade C)

\section{Good practice point}

- In patients with clinical evidence of peripheral oedema, polycythaemia (haematocrit $\geq 55 \%$ ) or pulmonary hypertension, referral for LTOT assessment may be considered at $\mathrm{SpO}_{2}$ levels $\leq 94 \%$ to identify patients with a resting $\mathrm{PaO}_{2} \leq 8 \mathrm{kPa}$. $(\sqrt{ })$

\section{Referral for home oxygen at hospital discharge}

- Patients should undergo formal assessment for LTOT after a period of stability of at least 8 weeks from their last exacerbation. (Grade B)

\section{Good practice points}

- Patients who have borderline saturations (ie 93-94\%) should have their oxygen saturations monitored at their annual review with their general practitioner (GP) or practice nurse, or sooner if they experience an exacerbation in the interim. $(\sqrt{ })$

- Patients who exacerbate frequently and are unable to achieve a period of stability lasting 8 weeks may need to be assessed at an earlier stage after exacerbation. If LTOT is ordered for such patients, they should be counselled that in the future LTOT may no longer be required once they achieve a more stable state. $(\sqrt{ })$

- Patients should not normally have LTOT ordered at the time of an acute exacerbation of their underlying condition. However, if home oxygen is ordered (eg, at hospital discharge), it should be limited to patients with an $\mathrm{SpO}_{2}$ of $\leq 92 \%$, who are breathless, and unable to manage off oxygen. These patients should undergo a blood gases assessment and be counselled that in the future LTOT may not be required after formal reassessment. $(\sqrt{ })$

- The date of the patient's last exacerbation should be included in the referral request to the home oxygen assessment service. $(\sqrt{ })$

Use of pulse oximetry, arterial and capillary blood gases in assessment for LTOT

- Patients potentially requiring LTOT should not be assessed using pulse oximetry alone. (Grade D)

Assessment using arterial blood gases and capillary blood gases

- Patients being assessed for LTOT should undergo initial assessment for suitability using arterial blood gases (ABG) sampling. (Grade A)

- Patients assessed for LTOT during a period of apparent clinical stability should undergo two ABG measurements at least 3 weeks apart, before the need for LTOT can be confirmed. (Grade B)

- Patients undergoing LTOT assessment should be reassessed with $\mathrm{ABG}$ after oxygen titration is complete to determine whether adequate oxygenation has been achieved without precipitating respiratory acidosis and/or worsening hypercapnia. (Grade D)
- For oxygen titration during LTOT assessment, capillary blood gases (CBG) sampling can be used in place of ABG sampling for re-measuring $\mathrm{PaCO}_{2}$ and $\mathrm{pH}$ at different oxygen flow rates. (Grade $\mathrm{A}$ )

- For oxygen titration during LTOT assessment, cutaneous capnography can be used in place of $A B G$ sampling for re-measuring $\mathrm{PaCO}_{2}$ alone but not $\mathrm{pH}$ at different oxygen flow rates. (Grade $\mathrm{A}$ )

\section{Good practice points}

- Patients undergoing a radial ABG should be assessed with an Allen's test first, to ensure they have a dual blood supply to the hand from both radial and ulnar arteries. $(\sqrt{ })$

- Patients undergoing a radial ABG should be consented for the procedure with a discussion of possible risks. $(\sqrt{ })$

- In many community commissioned home oxygen serviceassessment and review (HOS-AR) services it is not practical for patients to undergo ABG sampling during LTOT assessment. Under such circumstances, a combination of CBGs and oximetry (but not capnography) could be used as an alternative tool for initial assessment for LTOT, and after oxygen titration is complete. Some patients may receive LTOT unnecessarily using this approach, but it is unlikely that any patient would be inappropriately denied LTOT. $(\sqrt{ })$

\section{Management of hypercapnia during LTOT assessment}

- Patients with baseline hypercapnia should be monitored for the development of respiratory acidosis and worsening hypercapnia using ABGs after each titration of flow rate, as well as an ABG after oxygen titration is complete. (Grade D)

Good practice points

- Patients who develop a respiratory acidosis and/or a rise in $\mathrm{PaCO}_{2}$ of $>1 \mathrm{kPa}(7.5 \mathrm{~mm} \mathrm{Hg})$ during an LTOT assessment may have clinically unstable disease. These patients should undergo further medical optimisation and be reassessed after 4 weeks. $(\sqrt{ })$

- Patients who develop a respiratory acidosis and/or a rise in $\mathrm{PaCO}_{2}$ of $>1 \mathrm{kPa}(7.5 \mathrm{~mm} \mathrm{Hg})$ during an LTOT assessment on two repeated occasions, while apparently clinically stable, should only have domiciliary oxygen ordered in conjunction with nocturnal ventilatory support. $(\sqrt{ })$

\section{LTOT hours of use}

- LTOT should be ordered for a minimum of $15 \mathrm{~h}$ per day, and up to $24 \mathrm{~h}$ per day may be of additional benefit. (Grade C)

\section{LTOT flow rates}

- Patients eligible for LTOT should be initiated on a flow rate of $1 \mathrm{~L} / \mathrm{min}$ and titrated up in $1 \mathrm{~L} / \mathrm{min}$ increments until $\mathrm{SpO}_{2}$ $>90 \%$. An ABG should then be performed to confirm that a target $\mathrm{PaO}_{2} \geq 8 \mathrm{kPa}(60 \mathrm{~mm} \mathrm{Hg})$ at rest has been achieved. (Grade $\mathrm{B}$ )

- Non-hypercapnic patients initiated on LTOT should increase their flow rate by $1 \mathrm{~L} / \mathrm{min}$ during sleep in the absence of any contraindications. (Grade B)

- Patients initiated on LTOT who are active outdoors should receive an ambulatory oxygen assessment to assess whether their flow rate needs increasing during exercise. (Grade B)

\section{Good practice points}

- Ambulatory and nocturnal oximetry may be performed to allow more accurate flow rates to be ordered for exercise and sleep, respectively. $(\sqrt{ })$ 
- Patients initiated on LTOT who have cognitive, visual or coordination impairments, may not be able to safely manipulate their own flow rates and should be maintained on a single flow rate. $(\sqrt{ })$

- Flow rates may be increased at 20 min intervals during an oxygen titration until a target $\mathrm{PaO}_{2}$ is achieved. $(\sqrt{ })$

Patient education at time of assessment

- Patients initiated on LTOT should be provided with formal education by a specialist home oxygen assessment team to ensure compliance with therapy. (Grade D)

- Patients being commenced on home oxygen on discharge from hospital should be advised that home oxygen may be removed if reassessment shows clinical improvement. (Grade D)

\section{Follow-up of LTOT patients}

- LTOT patients should receive follow-up at 3 months after LTOT has been ordered, which should include assessment of blood gases and flow rate to ensure LTOT is still indicated and therapeutic. (Grade A)

- LTOT patients should receive follow-up visits at 612 months after their initial 3-month follow-up, which can be either home based or in combination with hospital visits. (Grade D)

- Follow-up visits should be conducted by a specialist home oxygen assessment team with the necessary skills to deliver patient education and manage withdrawal of home oxygen. (Grade D)

\section{Good practice point}

- All patients for whom LTOT has been ordered should be visited at home within 4 weeks by a specialist nurse or healthcare professional with experience of domiciliary oxygen therapy. The visit provides an opportunity to highlight potential risks and should be used to reinforce education and offer support to the patient and carer. Compliance may be checked, along with smoking status, symptoms of hypercapnia and oxygen saturations on oxygen to check that oxygen is therapeutic. $(\sqrt{ })$

\section{Nocturnal oxygen therapy}

- Nocturnal oxygen therapy (NOT) is not recommended in patients with COPD who have nocturnal hypoxaemia but who fail to meet the criteria for LTOT. (Grade A)

\section{Good practice point}

- Other causes of nocturnal desaturation in COPD should be considered such as obesity hypoventilation, respiratory muscle weakness or obstructive sleep apnoea (OSA). $(\sqrt{ })$

NOT in patients with cardiac disease and nocturnal desaturation

- NOT can be ordered for severe heart failure patients who do not fulfil indications for LTOT and have evidence of sleep disordered breathing (SDB) leading to daytime symptoms, after other causes of nocturnal desaturation have been excluded (eg, obesity hypoventilation or OSA) and heart failure treatment has been optimised. Treatment with modalities of ventilatory support should also be considered. (Grade B)

\section{Good practice point}

- If NOT is ordered for patients with severe heart failure, it should be ordered at a low flow rate of $1-2 \mathrm{~L} / \mathrm{min}$ and response should be assessed by a reduction in symptoms of daytime sleepiness, and SDB indices as measured by an overnight oximetry study. A blood gas assessment should be undertaken to exclude worsening hypercapnia and respiratory acidosis. Treatment with modalities of ventilatory support should be considered for patients who are hypercapnic. $(\sqrt{ })$

NOT in patients with CF

- NOT should not be given to patients with CF with nocturnal hypoxaemia alone who do not fulfil LTOT criteria. It can be considered in patients with evidence of established ventilatory failure, where it should be given with NIV support. (Grade B)

NOT in patients with ILD

- NOT should not be given to patients with ILD with nocturnal hypoxaemia alone, who do not fulfil LTOT criteria. (Grade B)

NOT in patients with neuromuscular weakness

- Patients with neuromuscular weakness affecting respiratory muscles should not have NOT alone ordered. It can be considered in patients with evidence of established ventilatory failure, where it should be given with NIV support. (Grade B)

NOT in patients with OSA, obesity hypoventilation syndrome or overlap syndrome

- Patients with OSA, obesity hypoventilation syndrome (OHS) or overlap syndrome should not have NOT alone ordered. It can be considered in patients with evidence of established ventilatory failure, where it should be given with NIV support. (Grade D)

Ambulatory oxygen therapy

- AOT should not be routinely offered to patients who are not eligible for LTOT. (Grade B)

- AOT should not be routinely offered to patients already on LTOT. (Grade D)

- Ambulatory oxygen therapy (AOT) assessment should only be offered to patients already on LTOT if they are mobile outdoors. (Grade A)

- AOT should be offered to patients for use during exercise in a pulmonary rehabilitation programme or during an exercise programme following a formal assessment demonstrating improvement in exercise endurance. (Grade B)

\section{Good practice points}

- Patients started on AOT should be reviewed regularly. If AOT was started during an exacerbation or when unwell, an initial review at $4-6$ weeks to check it is still indicated is essential. $(\sqrt{ })$

- Home visits may be useful to identify problems with equipment or set-up. Further reviews should be carried out every 6 months when stable, or sooner if the patient's clinical status changes. $(\sqrt{ })$

- AOT therapy may offer patients with active lifestyles or active treatment regimens (eg, CF) additional benefits. All patients should be assessed for AOT in the context of their daily activity and therapies. $(\sqrt{ })$

- It is recognised that there may be some patients, for example with ILD and disabling breathlessness, who do not qualify for LTOT but who do desaturate on exercise who may benefit from AOT. Once all other medical interventions have been optimised, these patients could be considered for AOT following formal assessment and continued provision following demonstration of benefit and compliance. $(\sqrt{ })$

- Patients with high respiratory rates (common in CF and ILD) should receive AOT at a selected flow rate via a Venturi 
mask, which exceeds their peak tidal and exertional inspiratory flow, and be supplied with home oxygen equipment which is able to deliver the required high flow rates. $(\sqrt{ })$

- AOT may be offered to LTOT patients who could otherwise not achieve $15 \mathrm{~h}$ per day oxygen usage, or who are severely hypoxaemic and are too symptomatic to leave their house without supplemental oxygen but may need to do so, for example to attend their GP or hospital appointments. Formal assessment is not required in these circumstances. $(\sqrt{ })$

\section{Palliative oxygen therapy}

- Patients with cancer or end-stage cardiorespiratory disease who are experiencing intractable breathlessness should not receive treatment with $\mathrm{POT}$ if they are non-hypoxaemic or have mild levels of hypoxaemia above current LTOT thresholds $\left(\mathrm{SpO}_{2} \geq 92 \%\right)$. (Grade $\left.\mathrm{A}\right)$

- Patients with cancer or end-stage cardiorespiratory disease who are experiencing intractable breathlessness should receive assessment for a trial of treatment with opiates from an appropriately trained healthcare professional. (Grade A)

- Patients with cancer or end-stage cardiorespiratory disease who are experiencing intractable breathlessness should receive assessment for a trial of treatment with non-pharmacological treatments including fan therapy from an appropriately trained healthcare professional. (Grade D)

\section{Good practice point}

- POT may on occasion be considered by specialist teams for patients with intractable breathlessness unresponsive to all other modalities of treatment. In those instances, individual formal assessment of the effect of palliative oxygen on reducing breathlessness and improving quality of life should be made. $(\sqrt{ })$

\section{Short burst oxygen therapy}

- Short burst oxygen therapy (SBOT) should not be ordered for use prior to or following exercise in hypoxaemic or normoxic patients with COPD. (Grade A)

- SBOT should not be ordered on discharge from hospital for non-hypoxaemic patients with severe COPD. (Grade A)

Use of SBOT in cluster headache

- SBOT delivering high flow oxygen therapy $(12 \mathrm{~L} / \mathrm{min}$ via a non-rebreather mask) should be offered to treat acute attacks of cluster headache $(\mathrm{CH})$. (Grade A)

\section{Good practice point}

- Appropriate equipment will need to be provided in order to ensure delivery of high flow rate oxygen at $12 \mathrm{~L} / \mathrm{min}$ for $\mathrm{CH}$ using a non-rebreather mask. Patients will usually have warning of a $\mathrm{CH}$ attack, and so provision should be made for urgent $4 \mathrm{~h}$ installation of home oxygen, if available, rather than a permanent home supply being provided. $(\sqrt{ })$

\section{Equipment for home oxygen therapy}

- Oxygen concentrators should be used to deliver LTOT at flow rates of $4 \mathrm{~L} / \mathrm{min}$ or less. (Grade B)

- Portable oxygen should be delivered by whatever mode is best suited to the individual needs of the patient to increase the daily amount of oxygen used and activity levels in mobile patients. (Grade C)

\section{Good practice point}

- The type of portable device selected should balance patient factors with cost effectiveness, resources and safety. $(\sqrt{ })$
Oxygen delivery

- Nasal cannulae should be considered as the first choice of delivery device for patients requiring home oxygen therapy. As an alternative some patients may benefit from or prefer a Venturi mask system. (Grade D)

- Oxygen-conserving devices can be used in home oxygen patients requiring high flow rates to increase the time the cylinder will last. (Grade B)

\section{Good practice points}

- Venturi masks should be considered in patients in whom there are concerns about existing or developing hypercapnic respiratory failure, those with a high resting respiratory rate or those with cognitive problems. $(\sqrt{ })$

- Oxygen-conserving devices should be considered in patients who are active outside the home, following an ambulatory oxygen assessment. $(\sqrt{ })$

\section{Humidification}

- Humidification of home oxygen should not be ordered for non-tracheostomy patients. (Grade D)

\section{Good practice point}

- Patients receiving oxygen via a tracheostomy should receive humidified oxygen. $(\sqrt{ })$

\section{Carrying home oxygen}

- Less able patients should be offered wheeled devices or backpacks if assessment shows they improve ambulation and quality of life. (Grade B)

\section{Good practice point}

- When being transported in cars, cylinders should be secured either with a seat belt, or in the foot-well or car boot, possibly using a cylinder box. Liquid oxygen should always be transported in an upright position. A warning triangle may be displayed and insurance companies should be informed. $(\sqrt{ })$

Safety and home oxygen therapy

- Smoking cessation should be discussed and written education given to all patients prior to ordering home oxygen and at each subsequent review if the patient continues to smoke. (Grade C)

- Patients should be made aware in writing of the dangers of using home oxygen within the vicinity of any naked flame such as pilot lights, cookers, gas fires and candles. (Grade D)

- Patients and family members who continue to smoke in the presence of home oxygen should be warned of the associated dangers of smoking in the presence of oxygen. (Grade D)

Good practice points

- Safety should be a factor when making decisions regarding the ordering of oxygen. Education and written information should be provided to the patient and family or carers regarding the safe use of oxygen and its equipment. $(\sqrt{ })$

- The risks of prescribing oxygen to active smokers should be considered on a case-by-case basis: this should include a home visit to assess the patient's home situation, attitude toward risks and smoking behaviour. Home oxygen assessment services may decide not to prescribe home oxygen to smokers if the risks are in their judgement too high. Particular consideration needs to be given to risks to children and risks to neighbours in multiple occupancy dwellings. A risk assessment tool should be used, and the health professional who is undertaking the risk assessment may need to visit the home in conjunction with the local 
fire service and/or the oxygen contractor. Where there is reasonable doubt, the therapy should not be prescribed. $(\sqrt{ })$

- Patients who continue to smoke or live with other household smokers should be informed that the home oxygen order will be reviewed and evidence of increased risk may lead to withdrawal of home oxygen therapy. $(\sqrt{ })$

- Carbon monoxide monitoring and measuring urine cotinine may help identify those patients who continue to smoke. $(\sqrt{ })$

- Patients should be made aware that they should not use e-cigarettes and chargers within the vicinity of their home oxygen. $(\sqrt{ })$

- Oil-based emollients and petroleum jelly can support combustion in the presence of oxygen. Patients should be made aware that only water-based products should be used on the hands and face or inside the nose while using oxygen. $(\sqrt{ })$

- The oxygen supplier should be informed if the patient continues to smoke in order for the engineer to consider it in the home oxygen supplier risk assessment. $(\sqrt{ })$

- Patients and family or carers should be instructed not to remove the fire breaks or to change flow rate on their oxygen equipment. Only oxygen tubing and connections supplied by the oxygen company should be used. $(\sqrt{ })$

- The local fire service should be made aware of patients who are using oxygen at home and especially those who continue to smoke in order for a home safety assessment to be carried out. $(\sqrt{ })$

- Patients and carers should be aware that tubing should be checked on a regular basis and repositioned as necessary to ensure safety by preventing trips and falls. $(\sqrt{ })$

\section{INTRODUCTION}

The British Thoracic Society (BTS) Home Oxygen Guideline provides detailed evidence-based guidance for the use of home oxygen for patients out of hospital. Although the majority of evidence comes from the use of oxygen in patients with chronic obstructive pulmonary disease (COPD), the scope of the guidance includes patients with a variety of long-term respiratory illnesses and other groups in whom oxygen is currently ordered, such as those with cardiac failure, cancer and end-stage cardiorespiratory disease, terminal illness and cluster headache $(\mathrm{CH})$. It explores the evidence base for the use of different modalities of oxygen therapy and patient-related outcomes such as mortality, symptoms and quality of life. The guideline also makes recommendations for assessment and follow-up protocols, and risk assessments, particularly in the clinically challenging area of home oxygen users who smoke. The guideline development group is aware of the potential for confusion sometimes caused by the current nomenclature for different types of home oxygen, and rather than renaming them has adopted the approach of clarifying those definitions, and in particular emphasising what is meant by long-term oxygen therapy (LTOT) and palliative oxygen therapy (POT). The home oxygen guideline provides expert consensus opinion in areas where clinical evidence is lacking, and seeks to deliver improved prescribing practice, leading to improved compliance and improved patient outcomes, with consequent increased value to the health service.

\section{Target audience for the guideline}

This guideline is aimed at all healthcare practitioners who are involved in the care of patients who use home oxygen therapy: this will include primary care clinicians (general practitioners (GPs), and practice and district nurses), those working in community nursing or palliative care teams, integrated respiratory teams, home oxygen assessment services and hospital specialist teams in respiratory medicine, cardiology, neurology, oncology, geratology and palliative care.

\section{Groups covered}

The home oxygen guideline addresses the use of home oxygen in adults with

- chronic respiratory disease including COPD, pulmonary hypertension, pulmonary vascular disease, cystic fibrosis (CF), interstitial lung disease (ILD), chest wall disease, neuromuscular disease, and pulmonary malignancy

- cardiac disease including congestive cardiac failure and adult congenital heart disease

- $\mathrm{CH}$.

It will also consider special situations including:

- palliative and end-of-life care

- patients discharged from hospital pending a formal assessment when stable

- smokers.

\section{Scope of the guideline}

The guideline considers the evidence base and makes recommendations for the use or restricted use of the following types of home oxygen therapy:

- long-term oxygen therapy (LTOT)

- nocturnal oxygen therapy (NOT)

- ambulatory oxygen therapy (AOT)

- palliative oxygen therapy (POT)

- short burst oxygen therapy (SBOT).

The guideline considers the evidence base and makes recommendations for referral, assessment (including the roles of oximetry, arterial blood gases (ABGs) and capillary blood gases (CBGs)), and follow-up of patients for home oxygen therapy. The guideline reviews the different equipment used to deliver home oxygen therapy.

Finally, the guideline reviews safety issues around home oxygen therapy, in particular risks of fire, burns and smoke inhalation from flammable sources such as smoking. It outlines the risk assessment processes which were put in place by the National Framework Agreement for home oxygen therapy (2010) which outlined responsibilities for home oxygen providers.

\section{Areas not covered by the guideline}

The guideline development group was aware of existing BTS guidelines in related areas and the following areas therefore fall outside the scope of this guideline:

- home oxygen in children (younger than 18)-home oxygen in children remains as a separate guideline. ${ }^{1}$

- home oxygen use during acute exacerbations of respiratory disease-this is covered by the BTS Guideline for Emergency Oxygen Use in Adult Patients. ${ }^{2}$

- home oxygen use during air travel—see the 2011 BTS guideline on recommendations for managing passengers with stable respiratory disease planning air travel..$^{3}$

The guideline development group were unable to cover all disease groups individually, for example bronchiectasis and asthma among others. In these areas no disease specific evidence for oxygen use was found.

\section{Methodology}

This guideline is based on the best available evidence. The methodology used to write the guideline adheres strictly to the 
criteria as set by the AGREE collaboration, which is available online (http://www.agreetrust.org/resource-centre/agree-ii/). The BTS Standards of Care Committee (SOCC) guideline production manual is available at http://www.brit-thoracic.org.uk/ guidelines-and-quality-standards/

\section{Clinical questions and literature search}

Clinical questions were structured in the PICO (Patient, Intervention, Control, Outcome) format (see online supplementary appendix 9) to define the scope of the guideline and inform the literature search.

Systematic electronic database searches were conducted in order to identify potentially relevant studies for inclusion in the guideline. For each topic area the following databases were searched: Ovid MEDLINE (including MEDLINE In-Process), Ovid EMBASE, and the Cochrane Library (including the Cochrane Database of Systematic Reviews and the Database of Abstracts of Reviews of Effects) from 1980.

The searches were first run in July 2012 and updated in January 2014 (see online supplementary appendix 10 for the search strategy). Searches included a combination of indexed terms and free text terms and were limited to English language publications only. The initial search identified 1392 potential abstracts and the second search 326 abstracts.

\section{Appraisal of the literature}

Appraisal was performed to be compliant with the AGREE collaboration. Four individuals $(\mathrm{MH}, \mathrm{SH}, \mathrm{TW}, \mathrm{JS})$ read the title and abstract of each article retrieved by the literature searches and decided whether the paper was definitely relevant, possibly relevant or not relevant to the project. Criteria formulated for categorising the abstracts into these three groups were:

- whether the study addressed the clinical question;

- whether the appropriate study type was used to produce the best evidence to answer the clinical question;

- review articles were excluded;

- the abstract was in English;

- abstracts were not rejected on the basis of the journal of publication, country in which the research was performed or published, or the date of publication.

The full paper was obtained for all relevant or possibly relevant abstracts and allocated to the relevant section(s) of the guideline.

The first screening process identified 511 of the initial 1392 reference abstracts to be definitely or possibly relevant to the guideline. Two guideline reviewers per section independently reviewed the abstracts to identify papers to be appraised for the guideline. The two reviewers for each section then independently appraised each paper assigned to them using the Scottish Intercollegiate Guidelines Network (SIGN) critical appraisal checklists. The reliability of the evidence in each individual study was graded using the SIGN critical appraisal check lists and is shown in the evidence tables $(++,+$ or - ) (see online supplementary appendix 11). The body of evidence for each recommendation was summarised into evidence statements and graded using the SIGN grading system (see table 1 ).

Disagreements were resolved by discussion with the section partner. The second literature search in January 2014 yielded 326 abstracts. Of these, 56 were identified as definitely or possibly relevant to the guideline. However, all of the pertinent abstracts from this search had been identified by the guideline development group (GDG) in the meantime and already incorporated.

\section{Table 1 Key to evidence statements}

\begin{tabular}{|c|c|}
\hline Grade & Evidence \\
\hline $1++$ & $\begin{array}{l}\text { High quality meta-analyses, systematic reviews of RCTs, or RCTs with a } \\
\text { very low risk of bias }\end{array}$ \\
\hline $1+$ & $\begin{array}{l}\text { Well conducted meta-analyses, systematic reviews of RCTs, or RCTs } \\
\text { with a low risk of bias }\end{array}$ \\
\hline $1-$ & $\begin{array}{l}\text { Meta-analyses, systematic reviews of RCTs, or RCTs with a high risk of } \\
\text { bias }\end{array}$ \\
\hline $2++$ & $\begin{array}{l}\text { High quality systematic reviews of case-control or cohort studies or } \\
\text { high quality case-control or cohort studies with a very low risk of } \\
\text { confounding, bias or chance and a high probability that the relationship } \\
\text { is causal }\end{array}$ \\
\hline $2+$ & $\begin{array}{l}\text { Well conducted case-control or cohort studies with a low risk of } \\
\text { confounding, bias or chance and a moderate probability that the } \\
\text { relationship is causal }\end{array}$ \\
\hline $2-$ & $\begin{array}{l}\text { Case-control or cohort studies with a high risk of confounding, bias or } \\
\text { chance and a significant risk that the relationship is not causal }\end{array}$ \\
\hline 3 & Non-analytic studies, for example case reports, case series \\
\hline 4 & Expert opinion \\
\hline
\end{tabular}

\section{Considered judgement and grading of evidence}

The GDG used the evidence tables to judge the body of evidence and grade recommendations for this guideline. Evidence tables are available in the online supplementary appendix 11. Where evidence was lacking to answer the formulated clinical questions, expert opinions were obtained through consensus. The following were considered in grading of the recommendations:

- the available volume of the body of evidence;

- how applicable the obtained evidence was in making recommendations for the defined target audience of this guideline;

- whether the evidence was generalisable to the target population for the guideline;

- whether there was clear consistency in the evidence obtained to support recommendations;

- what the implications of recommendations would be on clinical practice in terms of resources and skilled expertise;

- cost-effectiveness was not reviewed in detail as in-depth economic analysis of recommendations falls beyond the scope of this guideline.

Recommendations were graded from A to D as indicated by the strength of the evidence as shown in table 2. In line with SIGN guidance, evidence rated 'minus' was considered by the GDG in context but in the absence of other supporting evidence with a "plus" rating, any recommendation made was Grade D. Important practical points lacking any research evidence and not likely to be research evidence in the future, were highlighted as 'good practice points'.

\section{Drafting the guideline}

The GDG corresponded regularly by email and meetings of the full group were held in November 2011, February and November 2012, and March, April and September 2013 in addition to a number of teleconferences. The BTS SOCC reviewed the draft guideline in March 2014. The draft guideline was made available online in July/August 2014 for public consultation and circulated to all the relevant stakeholders. The BTS SOCC re-reviewed the revised draft guideline in December 2014 and final SOCC approval was granted in January 2015.

This BTS guideline will be reviewed within the next 5 years. 
Table 2 Grades of recommendations

\begin{tabular}{l} 
Grade $\quad$ Type of evidence \\
\hline A $\quad \begin{array}{l}\text { At least one meta-analysis, systematic review or RCT rated as } 1++ \text { and } \\
\text { directly applicable to the target population or } \\
\text { A systematic review of RCTs or a body of evidence consisting principally } \\
\text { of studies rated as } 1+\text { directly applicable to the target population and } \\
\text { demonstrating overall consistency of results }\end{array}$ \\
A body of evidence including studies rated as $2++$ directly applicable to \\
the target population and demonstrating overall consistency of results \\
or \\
Extrapolated evidence from studies rated as $1++$ or 1+ \\
A body of evidence including studies rated as $2+$ directly applicable to \\
the target population and demonstrating overall consistency of results \\
or \\
Extrapolated evidence from studies rated as $2++$ \\
Evidence level 3 or 4 or \\
Extrapolated evidence from studies rated as $2+$ \\
Important practical points for which there is no research evidence, nor \\
is there likely to be any research evidence. The guideline committee \\
wishes to emphasise these as Good Practice Points.
\end{tabular}

\section{Declarations of interest}

All members of the GDG made declarations of interest in line with BTS policy and further details can be obtained on request from BTS. GDG members are listed in appendix 8 .

\section{Acknowledgements}

The guideline development group members are grateful to:

- Wirral NHS home oxygen assessment service for the example of a Home Oxygen Risk Assessment form (see appendix 4);

- Oxford Health Foundation Trust home oxygen assessment service for an example of an Ambulatory Oxygen Assessment on which appendix 1 is based;

- The British Lung Foundation for patient information leaflets referred to in appendix 6;

- Barema (the Association for Anaesthetic and Respiratory Device Suppliers) and BOC Healthcare for the photographs of home oxygen equipment used to illustrate online supplementary appendix 12 .

\section{Audit and research recommendations}

1. Research to investigate which patients with particular disease phenotypes benefit from LTOT: for example smokers compared with ex-smokers, those with pulmonary hypertension, those with COPD-driven cachexia and frequent exacerbators.

2. Research to investigate long-term outcomes (survival) in diseases other than COPD such as CF, ILD and bronchiectasis.

3. Research to investigate delivery of oxygen during pulmonary rehabilitation and maintenance classes, assessing impact on outcomes such as exacerbations, exercise tolerance and quality of life.

4. Longitudinal studies to assess the impact of LTOT on pulmonary haemodynamics in COPD patients with pulmonary hypertension using both direct (eg, cardiac catheterisation) and indirect (eg, NT-proBNP, echocardiography) parameters, along with quality of life and exercise tolerance outcomes.

5. A robust assessment of risk assessment measures with the aim of developing an integrated pathway for home oxygen teams and oxygen provider services to manage patients who smoke.
6. Research to investigate the role of palliative oxygen in comparison with or used together with other measures such as opiates, fan therapy and cognitive behavioural therapy.

7. Research to investigate and compare the use of $A B G$ and CBG in predicting need for LTOT and risk of hypercapnia.

8. Audit of assessment, ordering for and follow-up of home oxygen patients to improve and maintain standards of care from home oxygen assessment teams.

\section{Glossary/Abbreviations and symbols}

\begin{tabular}{|c|c|}
\hline \multicolumn{2}{|c|}{ Abbreviations } \\
\hline$A B G$ & Arterial blood gas \\
\hline $\mathrm{AHI}$ & Apnoea hypopnoea index \\
\hline AOT & Ambulatory oxygen therapy \\
\hline ASV & Adaptive servo ventilation \\
\hline BIPAP & Bi-level positive airway pressure \\
\hline BTS & British Thoracic Society \\
\hline CBG & Capillary blood gas \\
\hline CCF & Congestive cardiac failure \\
\hline $\mathrm{CCH}$ & Chronic cluster headache \\
\hline $\mathrm{CF}$ & Cystic fibrosis \\
\hline $\mathrm{CH}$ & Cluster headache \\
\hline $\mathrm{CO}$ & Carbon monoxide \\
\hline $\mathrm{CO}_{2}$ & Carbon dioxide \\
\hline COPD & Chronic obstructive pulmonary disease \\
\hline CPAP & Continuous positive airway pressure \\
\hline CRQ & Chronic respiratory disease questionnaire \\
\hline CSA & Central sleep apnoea \\
\hline CSB & Cheyne-stokes breathing \\
\hline $\mathrm{ECH}$ & Episodic cluster headache \\
\hline ECOG PS & Eastern Cooperative Oncology Group Performance Status \\
\hline EEG & Electroencephalography \\
\hline ELBG & Earlobe blood gas \\
\hline ESS & Epworth sleepiness scale \\
\hline $\mathrm{FiO}_{2}$ & Fraction of inspired oxygen \\
\hline GDG & Guideline development group \\
\hline GP & General practitioner \\
\hline $\mathrm{H}$ & Hypoxaemia \\
\hline HAD & Hospital anxiety and depression scale \\
\hline $\mathrm{Hb}$ & Haemoglobin \\
\hline HO & Home oxygen \\
\hline HOOF & Home oxygen order form \\
\hline HOS-AR & Home oxygen service - assessment and review \\
\hline IPAH & Idiopathic Pulmonary Arterial Hypertension \\
\hline IOT & Intermittent oxygen therapy \\
\hline ILD & Interstitial lung disease \\
\hline $\mathrm{kPa}$ & kilo Pascal (unit of measurement of pressure) $1 \mathrm{kPa}=7.5 \mathrm{mmHg}$ \\
\hline $\mathrm{L} / \mathrm{min}$ & Litres per minute (unit of measure of flow rate of oxygen) \\
\hline LTOT & Long term oxygen therapy \\
\hline LVEF & Left ventricular ejection fraction \\
\hline $\mathrm{m}$ & Meter (unit of measurement of length) \\
\hline $\mathrm{mmHg}$ & millimetres of mercury (unit of measurement of pressure) \\
\hline MMSE & Mini mental state examination \\
\hline MQoLQ & Migraine quality of life questionnaire \\
\hline MRC & Medical Research Council \\
\hline $\mathrm{NH}$ & Non hypoxaemic \\
\hline NHYA & New York Heart Association \\
\hline NIV & Non-invasive ventilation \\
\hline NIPPV & Non-invasive positive pressure ventilation \\
\hline NOT & Nocturnal oxygen therapy \\
\hline NRS & Numeric rating scale \\
\hline
\end{tabular}




\begin{tabular}{|c|c|}
\hline $\mathrm{OHS}$ & Obesity Hypoventilation Syndrome \\
\hline ОТ & Oxygen therapy \\
\hline OSA & Obstructive sleep apnoea \\
\hline $\mathrm{O}_{2}$ & Oxygen \\
\hline PAP & Pulmonary artery pressure \\
\hline $\mathrm{PCO}_{2}$ & Carbon dioxide tension (partial pressure) in blood or alveolus \\
\hline $\mathrm{PaCO}_{2}$ & Arterial carbon dioxide tension (partial pressure) \\
\hline $\mathrm{PaO}_{2}$ & Arterial oxygen tension (partial pressure) \\
\hline $\mathrm{PO}_{2}$ & Oxygen tension (partial pressure) in blood or alveolus \\
\hline PCU & Palliative care unit \\
\hline PICO & Patient Intervention Control Outcome \\
\hline РОТ & Palliative oxygen therapy \\
\hline PPH & Primary pulmonary hypertension \\
\hline $\mathrm{pH}$ & Unit of measurement of acidity of blood \\
\hline QoL & Quality of life \\
\hline REM & Rapid eye movement stage of sleep \\
\hline SBOT & Short burst oxygen therapy \\
\hline SD & Standard deviation \\
\hline SDB & Sleep disorder breathing \\
\hline SF-A & Validated sleep quality questionnaire \\
\hline SF 36 & Short form (36) health questionnaire \\
\hline SIGN & Scottish Intercollegiate Guideline Network \\
\hline $\mathrm{SaO}_{2}$ & $\begin{array}{l}\text { Arterial oxygen saturation measured by arterial blood gas } \\
\text { co-oximetry }\end{array}$ \\
\hline $\mathrm{SpO}_{2}$ & Arterial oxygen saturation measured by pulse oximetry \\
\hline SOCC & British Thoracic Society Standards of Care Committee \\
\hline VAS & Visual analogue scale \\
\hline VE & Minute ventilation \\
\hline $6 \mathrm{MWT}$ & 6 minute walk test \\
\hline \multicolumn{2}{|c|}{ Symbols } \\
\hline$>$ & Greater than or above \\
\hline$\leq$ & Less than or below \\
\hline$>$ & Greater than or equal to \\
\hline$\leq$ & Less than or equal to \\
\hline$\%$ & Percent \\
\hline
\end{tabular}

\section{Partial pressure units of measurement and conversion} between them

- Partial pressures of oxygen and carbon dioxide are measured using kilopascals $(\mathrm{kPa})$ and millimetres of mercury $(\mathrm{mm} \mathrm{Hg})$ where:

- $1 \mathrm{kPa}=7.5 \mathrm{~mm} \mathrm{Hg}$, and $1 \mathrm{~mm} \mathrm{Hg}=0.133 \mathrm{kPa}$.

\section{LONG-TERM OXYGEN THERAPY}

LTOT can be defined as oxygen used for at least $15 \mathrm{~h}$ per day in chronically hypoxaemic patients. Chronic hypoxaemia is defined as a $\mathrm{PaO}_{2} \leq 7.3 \mathrm{kPa}$ or, in certain clinical situations, $\mathrm{PaO}_{2} \leq 8.0 \mathrm{kPa}$. LTOT is delivered via an oxygen concentrator and should be differentiated from the use of oxygen as a palliative measure for symptomatic relief in breathless patients, which will be discussed in the palliative oxygen therapy section. LTOT addresses specific physiological inclusion criteria as outlined below.

\section{Evidence for use of LTOT in patients with COPD}

\section{Survival benefit in COPD patients with LTOT}

Two landmark randomised controlled trials (RCTs) showed survival benefit of LTOT in patients with COPD and severe chronic hypoxaemia when used for at least $15 \mathrm{~h}$ daily.

The Nocturnal Oxygen Therapy Trial (NOTT) was the first RCT of LTOT in patients with COPD. ${ }^{4}$ It included 203 patients with COPD in six US centres with $\mathrm{PaO}_{2} \leq 7.33 \mathrm{kPa}(55 \mathrm{~mm} \mathrm{Hg})$, or $\mathrm{PaO}_{2}<7.87 \mathrm{kPa}(59 \mathrm{~mm} \mathrm{Hg})$ with a raised haematocrit, signs of right heart failure or P pulmonale. It compared the effects of $12 \mathrm{~h}$ nocturnal oxygen $(\mathrm{n}=102)$ therapy with continuous oxygen ( $24 \mathrm{~h} ; \mathrm{n}=101)$ therapy on mortality, pulmonary haemodynamics and exercise capacity at 12 months. The treatment groups were well matched. There was 1.94 times the mortality in the NOT group compared to the continuous oxygen therapy group. This survival benefit was present in relatively normocapnic patients, and in those without a raised pulmonary arterial pressure (PAP) but was more pronounced in patients with hypercapnia, severe airflow limitation, lower oxygen saturations and in those with neuropsychological impairment. There was also a correlation between the mean reduction in PAP in the first 6 months of LTOT and survival at 8-year follow-up.

The UK MRC (Medical Research Council) domiciliary oxygen trial studied 87 patients with chronic bronchitis and emphysema who were hypoxaemic $\left(\mathrm{PaO}_{2} 5.3-8.0 \mathrm{kPa}\right)$, who were mostly hypercapnic and who had a previous documented episode of oedema indicating cor pulmonale. Patients were randomised to no oxygen therapy or $15 \mathrm{~h} /$ day at an inspired oxygen concentration to achieve a $\mathrm{PaO}_{2}>8 \mathrm{kPa}{ }^{5}$ Over a 5 -year follow-up period in the MRC trial, 19/42 died in the LTOT (treatment) group compared with $30 / 45$ in the control (no oxygen) group.

Subsequent studies have confirmed a survival benefit of LTOT when given for at least $15 \mathrm{~h} /$ day in the presence of chronic hypoxaemia, irrespective of chronic hypercapnia or previous episodes of oedema or pulmonary hypertension. ${ }^{6-8}$ This survival benefit was not seen in patients with moderate hypoxaemia. No significant differences were found in survival rates between patients treated with LTOT and controls in a population of 135 patients with advanced airflow limitation (mean (SD) $\mathrm{FEV}_{1} 0.83$ $(0.28) \mathrm{L})$ and moderate hypoxaemia $\left(\mathrm{PaO}_{2} 7.4-8.7 \mathrm{kPa}, 56-65\right.$ $\mathrm{mm} \mathrm{Hg}$ ) followed up for at least 3 years or until death. ${ }^{9}$ Women have a worse prognosis on LTOT than men. ${ }^{10}$ Most patients treated with LTOT die as a result of respiratory failure. ${ }^{11}$ Nutritional depletion is an independent risk factor for mortality and hospitalisation in patients with COPD receiving LTOT. ${ }^{12}$

\section{Evidence for blood gas criteria for selection of} COPD patients for LTOT

Criteria for ordering LTOT and ABG parameters derive from the two previously described landmark RCTs. ${ }^{4} 5$ The NOTT trial included COPD patients with $\mathrm{PaO}_{2} \leq 7.33 \mathrm{kPa}$ $(\leq 55 \mathrm{~mm} \mathrm{Hg})$ or $\mathrm{PaO}_{2} \leq 8 \mathrm{kPa}(60 \mathrm{~mm} \mathrm{Hg})$ with a raised haematocrit, signs of right heart failure or $\mathrm{P}$ pulmonale on electrocardiogram. ${ }^{4}$ The UK MRC domiciliary oxygen trial studied outcomes in patients with chronic bronchitis and emphysema who were hypoxaemic $\left(\mathrm{PaO}_{2} 5.3-8 \mathrm{kPa}\right)$, mostly hypercapnic and who had a previous documented episode of oedema indicating cor pulmonale. ${ }^{5}$

\section{LTOT in hypercapnic COPD patients}

Few RCTs have directly studied the impact of providing oxygen by comparing a priori hypercapnic and normocapnic patients with COPD. In the MRC trial, an analysis of predictors of mortality demonstrated that raised red cell mass and baseline $\mathrm{PaCO}_{2}$ were predictors of mortality in both the treatment and placebo arms. ${ }^{5}$ Longitudinal analysis demonstrated that a rising $\mathrm{PaCO}_{2}$ and falling $\mathrm{PaO}_{2}$ were associated with poor outcomes in both arms. The authors concluded that there was no evidence of oxygen toxicity with this treatment regimen. In contrast, a study of 228 patients given an oxygen concentrator who were followed up for a maximum of 5 years, and analysed in three 
groups ( $n=55$, no use of oxygen; $n=112$, use for $<15$ h per day; and $n=61$, use for $>15 \mathrm{~h}$ per day) showed that median survival at 2 years was better in the groups receiving oxygen compared to the no use group. ${ }^{8}$ Baseline $\mathrm{PaCO}_{2}$ was not shown to be a predictor of mortality and did not predict differences in mortality between the groups. However, the NOTT trial showed the survival benefit in the treatment groups was more apparent in patients with hypercapnia. ${ }^{4}$

The effect of supplementary oxygen on the chemical control of ventilation has the potential to increase $\mathrm{CO}_{2}$ levels in patients receiving $24 \mathrm{~h} /$ day oxygen. Fleetham et al $^{13}$ studied 30 hypoxaemic COPD patients (mean $\mathrm{PaO}_{2} 6.9 \mathrm{kPa}$ ) who were randomised to 12 or $24 \mathrm{~h}$ oxygen therapy for 12 months. Patients given $24 \mathrm{~h}$ oxygen had a blunted $\mathrm{CO}_{2}$ response. There was no change in the hypoxaemic response in either group.

\section{Effects of LTOT on pulmonary haemodynamics in COPD patients} The effect of LTOT on PAP are small. In the NOTT trial, survival after 8 years was related to the decrease in mean PAP during the first 6 months of treatment. ${ }^{14}$ This subgroup analysis also showed improvement in PAP and stroke volume in patients with $24 \mathrm{~h}$ of oxygen therapy per day compared to those given only $12 \mathrm{~h}$ of oxygen per day. In the MRC trial, LTOT prevented a rise in PAP of $0.4 \mathrm{kPa}(3 \mathrm{~mm} \mathrm{Hg})$, seen in the control group, although a fall in PAP was not found. ${ }^{5}$ A small intervention study measured PAP and left ventricular ejection fraction (LVEF) before and after 6 months of LTOT and showed a significant fall in mean PAP. $^{15}$

\section{Effects of LTOT on sleep in COPD patients}

Patients with COPD can develop nocturnal hypoxaemia due to ventilation-perfusion mismatch, decreased functional capacity and nocturnal hypoventilation particularly pronounced during REM sleep. This in turn can lead to poor sleep quality with sleep fragmentation. Use of LTOT has been demonstrated to correct nocturnal $\mathrm{SaO}_{2}$, decrease sleep latency and improve sleep quality evaluated by EEG. ${ }^{16}$

Effects of LTOT on quality of life and neuropsychological function in COPD patients

Health-related quality of life is impaired in patients with COPD. In one study, the administration of LTOT showed no beneficial effects on quality of life compared to patients not fulfilling criteria for LTOT. ${ }^{17}$ In the NOTT study, minor improvements in neuropsychological function were achieved after 12 months of LTOT compared to NOT. ${ }^{18}$ There was only modest improvement in neuropsychological scores after 6 months of treatment. An observational study has shown improvement in mood after 1 year of treatment with LTOT. $^{19}$ However, psychological changes due to LTOT are difficult to separate from the effects of other therapies.

LTOT in COPD patients and impact upon hospital admissions Lack of provision of LTOT to hypoxaemic COPD patients with $\mathrm{PaO}_{2}<7.3 \mathrm{kPa}$ is an independent risk factor for hospital admission with a COPD exacerbation. ${ }^{20}$ Conversely, use of LTOT in moderately hypoxaemic patients $\left(\mathrm{PaO}_{2} 7.3-9.5 \mathrm{kPa}\right)$ does not significantly reduce hospital admission rates or bed days when comparing a 10-month period before and after LTOT treatment. $^{21}$ The MRC trial did not find any impact on hospitalisation from treatment with LTOT. ${ }^{5}$
Other effects of LTOT in COPD patients

A further benefit of LTOT may be in the improvement of renal blood flow, reducing activation of the renin angiotensin system and thus salt and water retention. However, one study of LTOT showed no overall benefit on renal function after 6 months of treatment. $^{22}$

Supplemental oxygen therapy reduces secondary polycythaemia, as seen by a fall in haematocrit and red cell mass. ${ }^{5}$ A study in COPD patients showed that LTOT patients with a low haematocrit have worse survival than patients with high haematocrits $(>0.55) .^{23}$

Evidence statements

- Patients whose clinical condition is stable with a resting $\mathrm{PaO}_{2}$ $\leq 7.3 \mathrm{kPa}$ have improved life expectancy when treated with LTOT for at least $15 \mathrm{~h} /$ day. Evidence level 1+

- Patients with stable COPD and a resting $\mathrm{PaO}_{2} \leq 8.0 \mathrm{kPa}$ with evidence of cor pulmonale, polycythaemia and/or pulmonary hypertension have improved outcomes with LTOT. Evidence level $1+$

- Use of continuous oxygen therapy $(24 \mathrm{~h})$ offers additional survival benefit compared to shorter durations (12-15 h) but can contribute to higher $\mathrm{PaCO}_{2}$ levels. Evidence level 1-

- Use of LTOT in hypercapnic respiratory patients with COPD does not lead to increased morbidity, mortality or healthcare utilisation. Evidence level 1+

Recommendations

- Patients with stable COPD and a resting $\mathrm{PaO}_{2} \leq 7.3 \mathrm{kPa}$ should be assessed for LTOT, which offers survival benefit and improves pulmonary haemodynamics. (Grade A)

- LTOT should be ordered for patients with stable COPD with a resting $\mathrm{PaO}_{2} \leq 8 \mathrm{kPa}$ with evidence of peripheral oedema, polycythaemia (haematocrit $\geq 55 \%$ ) or pulmonary hypertension. (Grade A)

- LTOT should be ordered for patients with resting hypercapnia if they fulfil all other criteria for LTOT. (Grade B)

\section{Evidence for use of LTOT in patients with other respiratory or cardiac disease \\ LTOT in patient with ILD}

Chronic hypoxaemia can occur in patients with severe ILD. As with other progressive respiratory conditions, the development of progressive hypoxaemia may lead to poor tissue oxygenation and the development of complications such as pulmonary hypertension. This in turn can worsen prognosis. However, there are no RCTs reporting the effects of use of LTOT in these disorders. Therefore, recommendations for use are extrapolated from evidence in COPD patients. In clinical practice, patients with severe breathlessness due to ILD may hyperventilate to maintain oxygen saturations, and often desaturate abruptly on minimal exertion. Clinical management varies, with some centres measuring oxygen saturation over a $24 \mathrm{~h}$ period to assess 'hypoxaemic burden' and prescribing home oxygen accordingly. There is at present no evidence to support home oxygen provision on this basis.

Evidence statement

- The use of LTOT in patients with ILD may improve survival and tissue oxygenation, and prevent complications associated with hypoxaemia such as worsening pulmonary hypertension. Evidence level 4

Recommendations

- LTOT should be ordered for patients with ILD with a resting $\mathrm{PaO}_{2} \leq 7.3 \mathrm{kPa}$. (Grade D)

- LTOT should be ordered for patients with ILD with a resting $\mathrm{PaO}_{2} \leq 8 \mathrm{kPa}$ in the presence of peripheral oedema, 
polycythaemia (haematocrit $\geq 55 \%$ ) or evidence of pulmonary hypertension. (Grade D)

Good practice point

- Patients with ILD who experience severe breathlessness could be considered for POT. $(\sqrt{ })$

\section{LTOT in patients with CF}

Patients with CF may develop chronic hypoxaemia with increasing severity of their disease. A Cochrane review examined 11 published studies of oxygen therapy in CF but no studies examined the use of LTOT. ${ }^{24}$ Recommendations for use are extrapolated from evidence in COPD patients.

Evidence statement

- The use of LTOT in patients with CF may improve survival and tissue oxygenation, and prevent complications associated with hypoxaemia such as worsening pulmonary hypertension. Evidence level 4

\section{Recommendations}

- LTOT should be ordered for patients with CF with a resting $\mathrm{PaO}_{2} \leq 7.3 \mathrm{kPa}$. (Grade D)

- LTOT should be ordered for patients with CF with a resting $\mathrm{PaO}_{2} \leq 8 \mathrm{kPa}$ in the presence of peripheral oedema, polycythaemia (haematocrit $\geq 55 \%$ ) or evidence of pulmonary hypertension. (Grade D)

\section{LTOT in patients with pulmonary hypertension}

Pulmonary hypertension may occur in a number of pulmonary vascular disorders such as idiopathic pulmonary arterial hypertension (IPAH), pulmonary arterial hypertension associated with portal hypertension, pulmonary arterial hypertension associated with connective tissues disease, drug-induced thromboembolism, pulmonary arterial hypertension and chronic thromboembolic pulmonary hypertension (CTEPH), which can all predispose to hypoxaemia. There is no evidence of the effectiveness of LTOT in RCTs in patients with pulmonary hypertension, with the exception of those patients who develop pulmonary hypertension as a complication of their COPD. Thus, use of LTOT in non-COPD patients with pulmonary hypertension is to improve tissue oxygenation and to prevent complications associated with hypoxaemia, such as worsening pulmonary hypertension, rather than to afford a specific survival benefit.

Evidence statement

- The use of LTOT in patients with pulmonary hypertension may improve tissue oxygenation and prevent complications associated with hypoxaemia rather than lead to a specific survival benefit. Evidence level 4

Recommendation

- LTOT should be ordered for patients with pulmonary hypertension, including idiopathic pulmonary hypertension, when the $\mathrm{PaO}_{2}$ is $\leq 8 \mathrm{kPa}$. (Grade D)

LTOT in patients with neuromuscular or chest wall disorders

Patients with chest wall disease (kyphoscoliosis, thoracoplasty) and neuromuscular disorders develop nocturnal hypoventilation, which causes nocturnal hypoxaemia and leads to chronic respiratory failure. Non-invasive ventilation (NIV) is the treatment of choice in these patients, although LTOT may be required additionally, particularly in patients with severe restrictive disease or where there is co-existing airways disease or obesity causing hypoxaemia which NIV alone does not correct (although there are no studies of this approach).

\section{Evidence statement}

- LTOT can be used in addition to NIV in patients with neuromuscular or chest wall disorders, particularly where there is co-existing airways disease or obesity causing hypoxaemia which NIV alone does not correct. Evidence level 4

Recommendation

- NIV should be the treatment of choice for patients with chest wall or neuromuscular disease causing type 2 respiratory failure. Additional LTOT may be required in case of hypoxaemia not corrected with NIV. (Grade D)

\section{LTOT in patients with advanced cardiac failure}

Some patients with advanced cardiac failure may have resting hypoxaemia although hypoxaemia is most consistently demonstrated during sleep in these patients. There are studies of NOT in patients with heart failure (see the section on nocturnal oxygen therapy) but no studies of the effects of LTOT in patients with chronic heart failure. The use of LTOT in patients with advanced cardiac failure and resting hypoxaemia may lead to improved tissue oxygenation and prevent complications associated with hypoxaemia such as worsening pulmonary hypertension.

Evidence statement

- The use of LTOT in patients with advanced cardiac failure and resting hypoxaemia may improve survival, tissue oxygenation and prevent complications associated with hypoxaemia. Evidence level 4

Recommendations

- LTOT should be ordered for patients with advanced cardiac failure with a resting $\mathrm{PaO}_{2} \leq 7.3 \mathrm{kPa}$. (Grade D)

- LTOT should be ordered for patients with advanced cardiac failure with a resting $\mathrm{PaO}_{2} \leq 8 \mathrm{kPa}$ in the presence of peripheral oedema, polycythaemia (haematocrit $\geq 55 \%$ ) or evidence of pulmonary hypertension on ECG or echocardiograph. (Grade D)

\section{Outcomes of LTOT in patients who continue to smoke}

Accurate reports of individual smoking status can be difficult to obtain reliably in clinical practice. All trial data around smoking come from trials conducted with COPD patients. $^{25}$ Unfortunately, the small numbers of patients included in the main RCTs is not optimal in discriminating between the impact of LTOT on smokers and non-smokers. ${ }^{4}$ There are no randomised or cohort studies investigating LTOT according to smoking status.

Cigarette smoking predisposes to secondary polycythaemia, accelerated decline in lung function and increased mortality in COPD. Thus, the beneficial effect of LTOT may be offset by raised carboxyhaemoglobin levels from continued cigarette smoking. ${ }^{26}$ The MRC study did not exclude smokers but did 'urge all patients to give up smoking'. 5 In the LTOT group, $52 \%$ of the patients were smokers (reduced to $44 \%$ at the end of the study). There were no recorded adverse events attributable to smoking in the MRC trial. There was an overall survival benefit in patients given oxygen (benefits in smoking and nonsmoking populations were not reported separately).

The significant risk associated with combining cigarette smoking and oxygen therapy is reviewed in a later safety section of the guideline.

Evidence statement

- Evidence is insufficient to determine adverse clinical outcomes related to the effect of continuing smoking in LTOT patients compared to non-smokers. Evidence level 2+ 


\section{Recommendation}

- If LTOT is ordered for patients who are continuing to smoke, the potential for more limited clinical benefit should be discussed with the patient. (Grade D)

\section{Referral and assessment of patients for LTOT}

Referral to home oxygen assessment services provides the appropriate means for patients to be assessed for home oxygen therapy. Patients should have a definite diagnosis and be medically optimised prior to referral for assessment by an oxygen service. These services should have the appropriate clinical expertise, equipment and access to appropriate support services to enable the patient to have the best available care and outcomes, with the best use of resources. Guidance for commissioners in England and Wales has been published. ${ }^{27}$

\section{Patient information on referral for home oxygen assessment}

In order to support a patient in understanding the implications of attending an assessment for home oxygen therapy, information is often given (whether verbal or written) at the time of referral. Failing to attend for initial assessment or reassessment could result in suboptimal treatment, poor clinical outcomes and wasted resources. There is a lack of published trial data on the impact of providing information on LTOT to patients in any format.

\section{Evidence statement}

- Provision of written and verbal information to patients at the point of referral to home oxygen assessment services can improve attendance at first referral. Evidence level 4

\section{Recommendation}

- Written and verbal information should be given to patients referred to home oxygen assessment services at the time of referral. (Grade D)

Use of oximetry as a screening tool for patient selection for LTOT Measurement of oxygen saturation using a pulse oximeter is widely available and presents a possible tool to be used for screening patients who might be candidates for LTOT. Studies have examined the use of an $\mathrm{SpO}_{2}$ value of $\leq 92 \%$ as a cut-off point at which patients will be deemed suitable for referral to an oxygen assessment service because of known evidence around ABG criteria for LTOT. Roberts et $a^{28}$ studied use of $\mathrm{SpO}_{2}$ levels alone or in combination with $\mathrm{FEV}_{1}$ in 113 COPD patients referred for LTOT, and showed that using an $\mathrm{SpO}_{2}$ level of $\leq 92 \%$ resulted in $100 \%$ sensitivity but a specificity of only $69 \%$ in identifying patients with a $\mathrm{PaO}_{2}<7.3 \mathrm{kPa}$. There was a particularly poor correlation between $\mathrm{SpO}_{2}$ values between $85 \%$ and $90 \%$ and ABGs. A study using pulse oximetry in screening patients in general practice for LTOT assessed 13 of 114 patients with a resting $\mathrm{SpO}_{2}$ of $\leq 92 \%$ and found three patients had a resting $\mathrm{PaO}_{2}<7.3 \mathrm{kPa}^{29}$ When Medicare guidelines for oxygen assessments were validated, an $\mathrm{SpO}_{2}$ level of $85 \%$ was found to still miss patients who would have required an oxygen assessment, but did demonstrate that at this level an oxygen assessment was not necessary for a large proportion of patients. ${ }^{30}$

Evidence statement

- An oxygen saturation $\left(\mathrm{SpO}_{2}\right)$ level of $\leq 92 \%$ can be used safely to identify patients for referral for LTOT. Evidence level $2+$

\section{Recommendation}

- Patients with a resting stable oxygen saturation $\left(\mathrm{SpO}_{2}\right)$ of $\leq 92 \%$ should be referred for a blood gas assessment in order to assess eligibility for LTOT. (Grade C)

\section{Good practice point}

- In patients with clinical evidence of peripheral oedema, polycythaemia (haematocrit $\geq 55 \%$ ) or pulmonary hypertension, referral for LTOT assessment may be considered at $\mathrm{SpO}_{2}$ levels $\leq 94 \%$ to identify patients with a resting $\mathrm{PaO}_{2} \leq 8 \mathrm{kPa}$. $(\sqrt{ })$

\section{Referral for home oxygen at hospital discharge}

It is recognised that an exacerbation of a cardiorespiratory condition may result in temporary worsening of hypoxaemia which may improve over time with recovery. However, the time course of recovery may be variable and undertaking an assessment for home oxygen prior to optimal treatment and recovery could result in the overprescribing of home oxygen and unnecessary repeated assessments for the patient. However, clinicians are frequently faced with the practical difficulty of managing patients who, having been treated with oxygen during the acute phase of their illness, feel they require oxygen in order to be discharged safely home. These patients are either normoxaemic at rest or remain hypoxaemic at the point of hospital discharge.

Several studies have looked at the timing of assessment for LTOT. In an RCT of 546 COPD patients allowing for a 2-month period of clinical stability rather than prescribing LTOT immediately after exacerbation, resulted in a $36 \%$ absolute difference in those given LTOT at 2 months, with about a $15 \%$ difference at 1 year. ${ }^{31}$ There was no significant difference in quality of life, mortality or use of community health resources between the two groups at 1 year. A subgroup analysis of the NOTT trial showed that $184 / 409(45 \%)$ patients in what was thought to be a clinically stable group on trial entry, subsequently improved their $\mathrm{PaO}_{2}$ to levels which excluded them from the trial after at least a 4-week follow-up period. ${ }^{32}$ Observational studies of home oxygen patients (the majority having COPD) who were supplied with LTOT from hospital discharge or during a period of clinical instability found that $30-58 \%$ of patients reassessed 1-3 months later no longer met the criteria for LTOT. ${ }^{33-35}$ In a study in which ABGs were measured monthly in 77 COPD patients following hospitalisation, improvements in levels of hypoxaemia were seen at each time point, with $30 \%$ of patients no longer meeting the criteria for LTOT at 4 months. ${ }^{36}$ Later withdrawal of LTOT if no longer required can lead to patient distress, and be challenging for staff to manage. ${ }^{37}$

No studies have defined criteria for safe discharge home from hospital without home oxygen pending a formal LTOT assessment.

Evidence statement

- Patients referred for LTOT assessment after an exacerbation of COPD can show improvement in hypoxaemia with recovery above the threshold for LTOT after an 8-week period. Evidence level 1+

\section{Recommendation}

- Patients should undergo formal assessment for LTOT after a period of stability of at least 8 weeks from their last exacerbation. (Grade B)

Good practice points

- Patients who have borderline saturations (ie, 93-94\%) should have their oxygen saturations monitored at their annual review with their GP or practice nurse, or sooner if they experience an exacerbation in the interim. $(\sqrt{ })$

- Patients who exacerbate frequently and are unable to achieve a period of stability lasting 8 weeks may need to be assessed at an earlier stage after exacerbation. If LTOT is ordered for such patients, they should be counselled that in the future LTOT may no longer be required once they achieve a more stable state. $(\sqrt{ })$ 
- Patients should not normally have LTOT ordered at the time of an acute exacerbation of their underlying condition. However, if home oxygen is ordered (eg, at hospital discharge), it should be limited to patients with an $\mathrm{SpO}_{2}$ of $\leq 92 \%$, who are breathless and unable to manage off oxygen. These patients should undergo a blood gases assessment and be counselled that in the future LTOT may not be required after formal reassessment. $(\sqrt{ })$

- The date of the patient's last exacerbation should be included in the referral request to the home oxygen assessment service. $(\sqrt{ })$

Use of pulse oximetry, ABGs and CBGs in assessment for LTOT Assessment using pulse oximetry alone

Measurement of oxygen saturations $\left(\mathrm{SaO}_{2}\right)$ provides information on the percentage of available haemoglobin that is combined with oxygen (ie, oxyhaemoglobin). The relationship between oxygen saturations and the $\mathrm{PO}_{2}$ in blood $\left(\mathrm{PaO}_{2}\right)$ is described by the oxyhaemoglobin dissociation curve. This curve is very steep once $\mathrm{PaO}_{2}$ falls below $8 \mathrm{kPa}(60 \mathrm{~mm} \mathrm{Hg})$ and thus small changes in $\mathrm{PaO}_{2}$ can greatly change oxygen saturationsthis characteristic allows the use of $\mathrm{SaO}_{2}$ to give estimates of $\mathrm{PaO}_{2}$ in hypoxaemic patients.

Whereas $\mathrm{SaO}_{2}$ is measured directly from blood using $\mathrm{CO}$-oximetry, pulse oximetry $\left(\mathrm{SpO}_{2}\right)$ measures oxygen saturations indirectly by comparing the absorbance of transmitted light before and during arterial pulsation at external sites such as earlobes or fingertips. This non-invasive tool carries a number of advantages as $\mathrm{SpO}_{2}$ can be measured rapidly with portable equipment by staff who are not necessarily skilled in arterial puncture. Conversely, the ability of $\mathrm{SaO}_{2}$ and $\mathrm{SpO}_{2}$ to estimate $\mathrm{PaO}_{2}$ is influenced by changes in the oxygen dissociation curve (eg, due to the presence of acidosis or changes in temperature), while oximetry alone cannot detect hypercapnia or acidosis.

Several studies have examined the use of pulse oximetry alone to determine LTOT requirement. In the largest study, 846 stable patients with chronic lung disease $(74.2 \%$ COPD) underwent LTOT assessment using both $\mathrm{SaO}_{2}$ (measured following ABG sampling) and $\mathrm{SpO}_{2}$ measurements. ${ }^{38} \mathrm{SpO}_{2}$ overestimated $\mathrm{SaO}_{2}$ in the presence of hypercapnia $\left(\mathrm{PaCO}_{2}>6.4 \mathrm{kPa}, 48 \mathrm{~mm} \mathrm{Hg}\right)$, while agreement between $\mathrm{SpO}_{2}$ and $\mathrm{SaO}_{2}$ was also poor under hypoxaemic conditions $\left(\mathrm{PaO}_{2}<7.2 \mathrm{kPa}, 54 \mathrm{~mm} \mathrm{Hg}\right)$. A smaller study of 55 stable patients with chronic lung disease and a resting $\mathrm{PaO}_{2}$ $<8.65 \mathrm{kPa}$ measured both $\mathrm{PaO}_{2}$ and $\mathrm{SpO}_{2}$ simultaneously on air at rest. ${ }^{30}$ Using $\mathrm{SpO}_{2}<88 \%$ as a threshold for prescribing LTOT would have led to $24-57 \%$ being denied LTOT and $7-21 \%$ being treated inappropriately, depending upon which brand of oximeter was used. Similar findings were reported from a study of 100 patients undergoing LTOT assessment, where using $\mathrm{SpO}_{2}$ alone with a $<88 \%$ threshold would have led to $56 \%$ of patients being inappropriately denied LTOT. ${ }^{35}$ No patient would have had LTOT ordered unnecessarily.

\section{Evidence statement}

- Pulse oximetry $\left(\mathrm{SpO}_{2}\right)$ agrees poorly with ABG CO-oximetry $\left(\mathrm{SaO}_{2}\right)$ and arterial oxygen tension $\left(\mathrm{PaO}_{2}\right)$ and cannot be used alone to assess the need for LTOT. Evidence level 3

\section{Recommendation}

- Patients potentially requiring LTOT should not be assessed using pulse oximetry alone. (Grade D)

\section{$A B G$ and $C B G$}

ABG sampling, performed via radial artery puncture, allows $\mathrm{PaO}_{2}, \mathrm{PaCO}_{2}$ and $\mathrm{pH}$ to be measured directly from arterial blood. Evidence for using ABGs to select patients for LTOT comes from previously reviewed trials. ${ }^{4} 36$ In the NOTT trial, subjects underwent ABG sampling on two occasions more than 1 week apart during a 3 -week observation period and were only recruited if they fulfilled the criteria of resting $\mathrm{PaO}_{2} \leq 7.33 \mathrm{kPa}$ $(55 \mathrm{~mm} \mathrm{Hg})$ or $\mathrm{PaO}_{2} \leq 7.86 \mathrm{kPa}(59 \mathrm{~mm} \mathrm{Hg})$ in the presence of one of oedema, haematocrit $\geq 55 \%$ or P pulmonale on ECG on both occasions. ${ }^{4}$ In the MRC trial, ABG measurements were repeated more than 3 weeks apart in stable patients who were included if their resting $\mathrm{PaO}_{2}$ was between $5.3 \mathrm{kPa}(40 \mathrm{~mm} \mathrm{Hg})$ and $8 \mathrm{kPa}(60 \mathrm{~mm} \mathrm{Hg})^{5}$

Although ABG sampling allows direct measurement of $\mathrm{PaO}_{2}$, it involves puncture of the radial artery and thus can be painful and can only be performed by trained healthcare professionals. There may be other considerations to take into account, such as a patient's past experience of ABG sampling and whether they are on anticoagulants. CBG sampling conversely only requires a small sample of blood $(125 \mu \mathrm{L})$ from a relatively superficial site (typically at the fingertip or earlobe). It is therefore less invasive, often better tolerated and can be performed by a wider range of healthcare professionals, although training and technique are still important to obtain adequately 'arterialised' samples. The difference in $\mathrm{PO}_{2}$ levels at an arterial level versus venous level can be significant, typically $8 \mathrm{kPa}(60 \mathrm{~mm} \mathrm{Hg})$ at rest and up to $10 \mathrm{kPa}(75 \mathrm{~mm} \mathrm{Hg})$ during exercise. ${ }^{39}$ To help raise capillary $\mathrm{PO}_{2}$ to a level closer to arterial $\mathrm{PO}_{2}$, a number of manoeuvres can be used prior to sampling, including the use of topical vasodilators and heat.

A meta-analysis included 886 subjects from 29 studies. $^{40}$ The studies included both healthy subjects and patients with chronic lung disease under a number of situations, including high altitude. Both earlobe CBGs and fingertip CBGs were compared against ABGs, and both gave accurate estimates of $\mathrm{pH}$ and $\mathrm{PaCO}_{2}$, although earlobe sampling gave a more accurate estimate for $\mathrm{PaCO}_{2}$. Earlobe CBGs were superior to fingertip CBGs in estimating $\mathrm{PaO}_{2}$ but continued to underestimate $\mathrm{PaO}_{2}$ by a mean of $0.32 \mathrm{kPa}(2.4 \mathrm{~mm} \mathrm{Hg})$ difference (1.9-2.8), residual SE $0.8 \mathrm{kPa}$ (6 mm Hg). The authors concluded that earlobe sampling gave a reasonable estimate of $\mathrm{PaO}_{2}$ unless precision was required.

In a comparison of simultaneous earlobe $\mathrm{CBG}$ and radial $\mathrm{ABG}$ from 40 patients with chronic lung disease, including 29 patients with COPD ${ }^{41}$ there was a good correlation between CBGs and ABGs for estimating $\mathrm{PaO}_{2}$, with CBGs underestimating $\mathrm{PaO}_{2}$ by a mean of just $0.17 \mathrm{kPa}$, albeit with a relatively wide $95 \% \mathrm{CI}$ $(-1.09 \mathrm{kPa}$ to $+0.75 \mathrm{kPa})$. A subgroup analysis suggested that CBGs were more accurate in hypoxaemic patients, with CBGs underestimating by $<0.5 \mathrm{kPa}$ in 'nearly all' patients with $\mathrm{PaO}_{2}$ $<8 \mathrm{kPa}$. In another study carrying out a comparison of simultaneous $\mathrm{PaO}_{2}$ and earlobe CBG measurements in 100 patients undergoing LTOT assessment, CBGs alone would have resulted in 9/55 (ie, 16\%) receiving LTOT inappropriately. No patients would have been denied LTOT. ${ }^{35}$ Conversely, patients found ABGs more uncomfortable than CBGs $(\mathrm{p}<0.0001)$.

A repeat $\mathrm{ABG}$ after oxygen titration is completed allows accurate reassessment of $\mathrm{PaO}_{2}, \mathrm{PaCO}_{2}$ and $\mathrm{pH}$, but can be uncomfortable for patients. Cutaneous capnography was used to reassess $\mathrm{PaCO}_{2}$ in comparison with $\mathrm{ABG}$ in 20 subjects with chronic lung disease who received oxygen at gradually increasing rates until $\mathrm{SaO}_{2}$ was $>90 \% .{ }^{42}$ Capnography accurately estimated $\mathrm{PaCO}_{2}$ with minimal bias. Earlobe CBGs have also been shown to give accurate estimates of $\mathrm{pH}$ and $\mathrm{PaCO}_{2}$ that are comparable to those achieved from ABGs. ${ }^{40}$

No studies were identified which showed that ABGs provided inaccurate results due to patient hyperventilation secondary to 
pain induced by the procedure. In clinical practice, many services routinely use local anaesthetic when performing radial ABGs. There are also no outcome data comparing complication rates between radial $\mathrm{ABG}$ s and earlobe CBGs.

Evidence statements

- ABG sampling, performed twice at least 3 weeks apart, during a stable phase of their condition, identifies patients who may benefit from LTOT. Evidence level $1++$

- Both earlobe and fingertip CBGs provide accurate estimates of arterial carbon dioxide tension and arterial $\mathrm{pH}$ during LTOT assessment and oxygen titration. Evidence level 1+

- Earlobe CBGs provide a more accurate estimate of arterial oxygen tension than fingertip CBGs. Evidence level 1+

- Use of earlobe CBGs alone for LTOT assessment leads to some patients inappropriately receiving LTOT. Evidence level 3

- Patients tolerate earlobe CBG testing better than ABG sampling. Evidence level 3

- During an LTOT assessment, cutaneous capnography can be used in place of $\mathrm{ABG}$ sampling for reassessing $\mathrm{PaCO}_{2}$ but not $\mathrm{pH}$ after oxygen titration. Evidence level 3

Recommendations

- Patients being assessed for LTOT should undergo initial assessment for suitability using ABG sampling. (Grade A)

- Patients assessed for LTOT during a period of apparent clinical stability should undergo two ABG measurements at least 3 weeks apart, before the need for LTOT can be confirmed. (Grade B)

- Patients undergoing LTOT assessment should be reassessed with ABG after oxygen titration is complete to determine whether adequate oxygenation has been achieved without precipitating respiratory acidosis and/or worsening hypercapnia. (Grade D)

- For oxygen titration during LTOT assessment, CBG sampling can be used in place of $\mathrm{ABG}$ sampling for re-measuring $\mathrm{PaCO}_{2}$ and $\mathrm{pH}$ at different oxygen flow rates. (Grade A)

- For oxygen titration during LTOT assessment, cutaneous capnography can be used in place of $\mathrm{ABG}$ sampling for re-measuring $\mathrm{PaCO}_{2}$ alone but not $\mathrm{pH}$ at different oxygen flow rates. (Grade $\mathrm{A}$ )

Good practice points

- Patients undergoing a radial ABG should be assessed with an Allen's test first, to ensure they have a dual blood supply to the hand from both radial and ulnar arteries. $(\sqrt{ })$

- Patients undergoing a radial ABG should be consented for the procedure with a discussion of possible risks. $(\sqrt{ })$

- In many community commissioned home oxygen serviceassessment and review (HOS-AR) services, it is not practical for patients to undergo ABG sampling during LTOT assessment. Under such circumstances, a combination of CBGs and oximetry (but not capnography) could be used as an alternative tool for initial assessment for LTOT, and after oxygen titration is complete. Some patients may receive LTOT unnecessarily using this approach, but it is unlikely that any patient would be inappropriately denied LTOT. $(\sqrt{ })$

\section{Management of hypercapnia during LTOT assessment}

Patients with chronic lung disease may develop resting hypercapnia as the severity of their disease progresses. In such cases, oxygen supplementation can cause suppression of existing hypoxaemic respiratory drive with consequent diminution of minute ventilation and worsening hypercapnia and V/Q mismatch. This has the potential to lead to the development of respiratory acidosis and progressive ventilatory failure.

Few studies exist to support best practice where patients became acidotic or excessively hypercapnoeic during an LTOT assessment. Neither the MRC $^{5}$ nor the NOTT ${ }^{4}$ studies excluded patients with hypercapnia. In the MRC study, average $\mathrm{PaCO}_{2}$ ranged between 7.1 and $7.3 \mathrm{kPa}(53.2-54.9 \mathrm{~mm} \mathrm{Hg})$ for both control and treated groups. Subjects received oxygen at $2 \mathrm{~L} / \mathrm{min}$ or higher if necessary to achieve $\mathrm{PaO}_{2}>8 \mathrm{kPa}(60 \mathrm{~mm} \mathrm{Hg})$, and no hypercapnia-related issues during the assessment process were reported. Although the NOTT study did not explicitly exclude patients with hypercapnia, patients in both groups were mostly normocapnoeic (mean $\mathrm{PaCO}_{2}$ $5.7 \mathrm{kPa}, 43 \mathrm{~mm} \mathrm{Hg}$ ). ${ }^{4}$ Again no hypercapnia-related issues during the assessment process were reported. Chiang et $a l^{43}$ studied ventilatory responses to $\mathrm{CO}_{2}$ stimulation in 26 COPD patients, 12 with resting hypercapnia, following oxygen supplementation with $2 \mathrm{~L} / \mathrm{min}$ oxygen. Hypercapnic patients showed a rise in mean $\mathrm{PCO}_{2}$ from $7.1 \pm 0.2 \mathrm{kPa}$ to $7.8 \pm 0.3 \mathrm{kPa}$ without developing acidosis and also a blunted response to $\mathrm{CO}_{2}$ stimulation. However, there were no reported adverse clinical events during this shortterm study.

Evidence statement

- Patients with baseline hypercapnia can undergo LTOT assessment without adverse outcome but require monitoring of $\mathrm{pH}$ and $\mathrm{PCO}_{2}$ levels during and at the end of assessment. Evidence level 4

\section{Recommendation}

- Patients with baseline hypercapnia should be monitored for the development of respiratory acidosis and worsening hypercapnia using ABGs after each titration of flow rate, as well as ABG sampling after oxygen titration is complete. (Grade D)

\section{Good practice points}

- Patients who develop a respiratory acidosis and/or a rise in $\mathrm{PaCO}_{2}$ of $>1 \mathrm{kPa}(7.5 \mathrm{~mm} \mathrm{Hg})$ during an LTOT assessment may have clinically unstable disease. These patients should undergo further medical optimisation and be reassessed after 4 weeks. $(\sqrt{ })$

- Patients who develop a respiratory acidosis and/or a rise in $\mathrm{PaCO}_{2}$ of $>1 \mathrm{kPa}(7.5 \mathrm{~mm} \mathrm{Hg})$ during an LTOT assessment on two repeated occasions, while apparently clinically stable, should only have domiciliary oxygen ordered in conjunction with nocturnal ventilatory support. $(\sqrt{ })$

Use of LTOT: hours of use and flow rates

\section{LTOT hours of use}

The benefits of LTOT are derived from normalisation of abnormal physiology driven by chronic hypoxaemia and have been achieved with use of LTOT for $15 \mathrm{~h} /$ day. ${ }^{5}$ Therefore, there is a hypothetical advantage of longer durations of oxygen therapy in correcting these abnormalities for greater periods of each day and particularly at night, when hypoxaemia may be more profound during sleep. Comparison of the effects of $12 \mathrm{~h}$ NOT with continuous oxygen $(24 \mathrm{~h})$ therapy in the NOTT study demonstrated a 1.94 times higher mortality in the NOT group: this survival benefit may be offset by the practicalities of increased oxygen use and the impact upon mobility. ${ }^{4}$

In a pragmatic 5-year follow-up study of 228 patients for whom an oxygen concentrator was ordered, comparisons were made between no oxygen use $(n=55)$, oxygen use for $<15 \mathrm{~h}$ per day $(n=112)$ and oxygen use for $>15$ h per day $(n=61)$. Overall survival at 2 years was better in the groups receiving oxygen compared to the no oxygen group, but there was no difference between the oxygen groups. ${ }^{8}$ 
There is no evidence base for duration of LTOT use in non-COPD respiratory disease or cardiac disease.

\section{Evidence statements}

- LTOT ordered for COPD patients for at least $15 \mathrm{~h}$ and up to $24 \mathrm{~h}$ per day confers a mortality benefit and improvement in physiological indices. Evidence level 1+

- Use of LTOT for $24 \mathrm{~h}$ versus $12 \mathrm{~h}$ offers additional benefits especially for COPD patients with more severe disease (higher $\mathrm{PaCO}_{2}$, higher haematocrit, higher pulmonary artery pressure and more neuropsychological impairment). Evidence level 2+

Recommendation

- LTOT should be ordered for a minimum of $15 \mathrm{~h}$ per day, and up to $24 \mathrm{~h}$ per day may be of additional benefit. (Grade C)

\section{LTOT flow rates}

\section{Daytime activity and LTOT flow rates}

A flow rate based on a single measure of oxygenation at rest may not necessarily guarantee adequate oxygenation during day-to-day activities where oxygen requirements may fluctuate. Although transient hypoxaemia could temporarily increase both pulmonary artery pressures and the risk of arrhythmias, it is unclear to what degree such fluctuations in oxygenation during daily life can offset the potentially beneficial effects of LTOT. Patients in the MRC study were started on a flow rate of $2 \mathrm{~L} / \mathrm{min}$, which was increased incrementally until a $\mathrm{PaO}_{2}>8 \mathrm{kPa}$ $(60 \mathrm{~mm} \mathrm{Hg})$ was achieved, ${ }^{5}$ and patients in the NOTT trial started on a flow rate of $1 \mathrm{~L} / \mathrm{min}$, which was increased in $1 \mathrm{~L} / \mathrm{min}$ increments up to a maximum of $4 \mathrm{~L} / \mathrm{min}$ until $\mathrm{PaO}_{2}>8 \mathrm{kPa}$ $(60 \mathrm{~mm} \mathrm{Hg})$ was achieved. ${ }^{4}$ Flow rates were not altered to reflect exercise. However, a number of studies suggest that determining flow rates using a single measure of $\mathrm{PaO}_{2}$ at rest may not guarantee adequate oxygenation during exercise: ${ }^{44-47}$ stable COPD patients receiving LTOT at a single flow rate spent between $70 \%$ and $87 \%$ of the daytime with $\mathrm{SpO}_{2}>90 \%$ when performing day-to-day activities. Individual tailoring of flow rates to suit patients' requirements during exercise, rest and sleep can reduce median oxygen flow rate from 2.5 to $1.2 \mathrm{~L} / \mathrm{min}$, while the percentage of time $\mathrm{SpO}_{2}$ was within the target range increased from $24.8 \%$ to $52.8 \%(\mathrm{p}=0.001){ }^{48}$

\section{Nocturnal oxygen requirements and LTOT}

Patients can desaturate during sleep as a result of reduced minute ventilation and impaired ventilatory responses and so oxygen requirements overnight may also differ from those at rest when awake. Several studies have suggested that a flow rate established from resting ABGs while awake may not allow adequate oxygenation overnight with patients spending only between $72 \%$ and $77 \%$ of the time with $\mathrm{SpO}_{2}>90 \%$ overnight. ${ }^{46} 4950$ Patients with more severe COPD and worse daytime ABGs spent a significantly greater proportion of the night with hypoxaemia. ${ }^{51}$ In the NOTT study, oxygen was automatically increased by $1 \mathrm{~L} / \mathrm{min}$ during sleep without reported adverse events. ${ }^{4}$ No data were found with respect to other diagnostic patient groups.

Evidence statements

- Patients for whom LTOT is ordered at a single flow rate sufficient to achieve $\mathrm{PaO}_{2}>8 \mathrm{kPa}(60 \mathrm{~mm} \mathrm{Hg})$ at rest demonstrate a survival benefit from LTOT. Evidence level 1+

- LTOT ordered at a single flow rate to provide adequate oxygenation at rest may offer inadequate oxygenation during exercise and/or sleep. Evidence level 3

- LTOT ordered for patients at different flow rates for use during sleep and exercise demonstrates a survival benefit from LTOT. Evidence level 1+

\section{Recommendations}

- Patients eligible for LTOT should be initiated on a flow rate of $1 \mathrm{~L} / \mathrm{min}$ and titrated up in $1 \mathrm{~L} /$ min increments until $\mathrm{SpO}_{2}$ $>90 \%$. An ABG should then be performed to confirm that a target $\mathrm{PaO}_{2} \geq 8 \mathrm{kPa}(60 \mathrm{~mm} \mathrm{Hg})$ at rest has been achieved. (Grade B)

- Non-hypercapnic patients initiated on LTOT should increase their flow rate by $1 \mathrm{~L} / \mathrm{min}$ during sleep in the absence of any contraindications. (Grade B)

- Patients initiated on LTOT who are active outdoors should receive an ambulatory oxygen assessment to assess whether their flow rate needs to increase during exercise. (Grade B)

Good practice points

- Ambulatory and nocturnal oximetry may be performed to allow more accurate flow rates to be ordered for use during exercise and sleep, respectively. $(\sqrt{ })$

- Patients initiated on LTOT who have cognitive, visual or coordination impairments, may not be able to safely manipulate their own flow rates and should be maintained on a single flow rate. $(\sqrt{ })$

- Flow rates may be increased at 20 min intervals during an oxygen titration until a target $\mathrm{PaO}_{2}$ is achieved. $(\sqrt{ })$

\section{Patient education at time of assessment}

A few studies have evaluated the provision of patient education, usually in the form of verbal or written information, at the time of oxygen assessment. A comparison of patients who had received formal assessment with ABGs on two separate occasions together with education by a specialist respiratory team with patients commencing LTOT in primary care, mostly on the basis of oximetry alone, demonstrated a significantly higher compliance ( $82 \%$ vs $44 \% ; p=0.002)$ and understanding of the rationale for treatment $(93 \%$ vs $41 \%$; $<<0.00001){ }^{52}$ These findings were supported by a large case series of 930 patients in whom education consisting of a home visit by a nurse or physiotherapist was an important factor in those patients' compliance with $\geq 15 \mathrm{~h} /$ day of oxygen use. ${ }^{53}$ Ordering LTOT on hospital discharge does not prepare patients for a follow-up assessment or the implications of oxygen removal if they no longer meet the criteria for LTOT: ${ }^{54}$ psychological dependence on oxygen therapy was reported as a major issue in these patients, causing distress for patients and staff as well as requiring significant resources and expertise to address.

Evidence statements

- Patients initiated on LTOT without formal education exhibit poor compliance with therapy. Evidence level 2+

- Providing written information to patients commenced on home oxygen in hospital does not prepare them for follow-up or the implication of not meeting the criteria for LTOT. Evidence level 3

Recommendations

- Patients initiated on LTOT should be provided with formal education by a specialist home oxygen assessment team to ensure compliance with therapy. (Grade D)

- Patients being commenced on home oxygen on discharge from hospital should be advised that home oxygen may be removed if reassessment shows clinical improvement. (Grade D)

\section{Follow-up of LTOT patients}

Follow-up of LTOT patients is necessary for a variety of reasons: to ensure that LTOT treatment is still required, that the oxygen order is still adequate (and therefore that the potential 
for healthcare gains such as survival are realised), that patients are compliant with treatment, and that any concerns or problems are addressed. Although home oxygen patients may be a relatively small group of lung disease patients in general, they are a very resource intensive group. Targeted follow-up of this group could provide significant benefits in terms of cost effective healthcare utilisation.

The original MRC and NOTT LTOT studies both provided titration of flow rate at a 3-month follow-up appointment as part of their protocol. ${ }^{45}$ Cottrell et $a l^{55}$ randomised 50 LTOT patients to follow-up at 2, 6 or 12 months. They costed hospital interventions given/required over a 1-year period, and found that the 2-monthly follow-up group had significantly higher evaluation costs with no benefit in terms of emergency department or hospital visits, length of stay in hospital or mortality: the only clinical benefit shown was an improvement in the psychological component of the sickness impact profile.

Other studies have focused on the setting for reassessment using different models, some of which might now be called 'integrated respiratory services'. Cross-sectional studies have reported 'added value' from reviewing patients in their homes, which included identifying and correcting problems with the concentrator, humidifier, the length of the patient's tubing and factors impacting on the patient's usage. ${ }^{56}{ }^{57} \mathrm{In}$ a prospective 10-year case-control study in which 217 LTOT patients were randomised to 'home care' (defined as 6-monthly hospital appointments and 2-3-monthly home visits) or standard care (management by hospital physician only), home care decreased exacerbation rates. ${ }^{58}$ Randomisation of 122 LTOT patients to follow-up with a hospital-based homecare programme (monthly phone call, home visits every 3 months, and home or hospital visits on a demand basis) or conventional medical care, demonstrated significantly decreased costs in the homecare follow-up group, which was mainly due to a reduction in use of hospital resources, despite the cost of running the service itself. ${ }^{59}$ Reports from focus groups ${ }^{60}$ and case series ${ }^{53}$ highlight the importance of education from specialist nurses or physiotherapists in increasing compliance and addressing patient concerns.

\section{Withdrawal of home oxygen}

Case series in which LTOT patients have been followed up have shown that a significant proportion of patients no longer required oxygen as originally ordered. ${ }^{33}{ }^{61}$ In addition, it has been well recognised that compliance with LTOT can be poor and that withdrawal of home oxygen through non-use is sometimes indicated. Withdrawal of LTOT can be distressing to patients, challenging for staff and entail a significant use of resources. ${ }^{37}$ See appendix 7 for a suggested protocol for withdrawal of home oxygen therapy.

Evidence statements

- Follow-up of LTOT patients 3 months after starting LTOT, can ensure that LTOT is still required and that the flow rate is appropriate. Evidence level 1+

- Six-monthly follow-up has a similar effect to 2-monthly follow-up in terms of healthcare utilisation but at decreased cost. Evidence level 3

- Home follow-up alone or in combination with hospital follow-up is more effective than hospital follow-up. Evidence level $2+$

- Follow-up with a specialist home oxygen assessment team including education improves compliance with LTOT. Evidence level 3

\section{Recommendations}

- LTOT patients should receive follow-up at 3 months after LTOT is ordered, which should include assessment of blood gases and flow rate to ensure LTOT is still indicated and therapeutic. (Grade A)

- LTOT patients should receive follow-up visits at 612 months after their initial 3-month follow-up, which can be either home based or in combination with hospital or clinic visits. (Grade D)

- Follow-up visits should be conducted by a specialist home oxygen assessment team with the necessary skills to deliver patient education and manage withdrawal of home oxygen. (Grade D)

\section{Good practice point}

- All patients for whom LTOT has been ordered should be visited at home within 4 weeks by a specialist nurse or healthcare professional with experience of domiciliary oxygen therapy. The visit provides an opportunity to highlight potential risks and should be used to reinforce education and offer support to the patient and carer. Compliance may be checked, along with smoking status, symptoms of hypercapnia and oxygen saturations on oxygen to check that oxygen is therapeutic. $(\sqrt{ })$

\section{NOCTURNAL OXYGEN THERAPY}

NOT is oxygen administered overnight alone without additional oxygen therapy during awake or daytime hours. Before daytime resting hypoxaemia develops, many patients develop nocturnal or sleep time oxygen desaturation due to a combination of worsening $\mathrm{V} / \mathrm{Q}$ mismatch in a supine posture and lack of drive to ventilatory muscles during sleep. This section refers to patients who are either normoxic during the day, or have mild daytime hypoxaemia but do not fulfil LTOT criteria.

\section{NOT in COPD patients with nocturnal desaturation}

The worsening of hypoxaemia during sleep in patients with advanced COPD has been well established in many studies. There is retrospective evidence that nocturnal desaturation is associated with worse survival. The evidence as to whether patients who fail to meet the criteria for LTOT but are hypoxaemic during sleep benefit from NOT is assessed here.

There are only a small numbers of studies addressing this population of patients. Of these, three studies examined outcomes over 2-3 years, and three studies looked at mortality. Patients were recruited from out-patients settings, largely in teaching hospitals. In a multicentre study, 76 patients with COPD (obstructive sleep apnoea (OSA) excluded) were identified as having nocturnal desaturation but did not qualify for LTOT. ${ }^{62} \mathrm{~A}$ total of 41 patients were randomised to receive NOT which was titrated to achieve saturations $>90 \%$ throughout the night. In the follow-up period, 22 patients went on to develop hypoxaemia requiring LTOT, 16 patients died (nine in the LTOT group), and there was no difference in the PAP (measured by cardiac catheter) between the two groups. A double-blind crossover study ${ }^{63}$ randomised 23 patients with COPD and nocturnal hypoxaemia to receive air or NOT over a 1-night period. No difference was seen in the quality of sleep (assessed by questionnaire and EEG) between the two groups. In six centres, 203 patients diagnosed with COPD and significant hypoxaemia $\left(\mathrm{PaO}_{2}<7.8 \mathrm{kPa}, 59 \mathrm{~mm} \mathrm{Hg}\right)$ were randomised to continuous oxygen therapy $(24 \mathrm{~h})$ or NOT $(12 \mathrm{~h}) .{ }^{4}$ The primary end point was all cause mortality. There was a 1.94 times increase in 'all cause' mortality in the NOT group compared to the continuous oxygen therapy group. A multicentre retrospective study investigated the data of patients from five centres who had polysomnography performed. ${ }^{64}$ Patients had mild to moderate daytime 
hypoxaemia with a $\mathrm{PaO}_{2}>60 \mathrm{mmg} \mathrm{Hg}(8 \mathrm{kPa})$ and evidence of desaturation during sleep without signs of sleep apnoea. A total of 169 subjects with COPD (77 desaturators and 92 nondesaturators) were analysed. The mean survival was significantly less in the desaturator group $(2.89 \pm 1.7$ years vs $3.7 \pm 1.7$ years; $\mathrm{p}<0.003)$. Thirty-five of the desaturator group were reported to have received some form of oxygen supplementation, however it was not clear how many hours this was for or if it was used nocturnally; on analysis it did not alter survival. In a doubleblind study of 51 patients with moderate COPD and daytime $\mathrm{PaO}_{2}>60 \mathrm{~mm} \mathrm{Hg}$ including 38 with desaturation, patients were allocated to receive NOT at $3 \mathrm{~L} / \mathrm{min}$ or room air. ${ }^{65}$ After 3 years, the NOT-treated group PAP had reduced by $0.49 \mathrm{kPa}$ $(3.7 \mathrm{~mm} \mathrm{Hg})$ and had increased in the air-treated group by $0.52 \mathrm{kPa}(3.9 \mathrm{~mm} \mathrm{Hg} ; \mathrm{p}<0.02)$. There was no difference in mortality; however, only nine in the sham group and seven in the oxygen group completed the study.

\section{Evidence statements}

- Patients with mild daytime hypoxaemia and nocturnal hypoxaemia have a worse survival compared to patients with no nocturnal desaturation. Evidence level 1+

- When administered to patients who are either normoxaemic or have baseline ABG levels above the threshold for LTOT, NOT alone does not show consistent improvements in pulmonary haemodynamics leading to a survival advantage. Evidence level 1+

- No additional significant benefit in sleep quality is derived from nocturnal supplemental oxygen in patients with nocturnal hypoxaemia. Evidence level 1-

\section{Recommendation}

- NOT is not recommended in patients with COPD who have nocturnal hypoxaemia but who fail to meet the criteria for LTOT. (Grade A)

Good practice point

- Other causes of nocturnal desaturation in COPD should be considered such as obesity hypoventilation, respiratory muscle weakness or OSA. $(\sqrt{ })$

\section{NOT in patients with cardiac disease and nocturnal desaturation}

A variety of factors can contribute to the development of nocturnal hypoxaemia in patients with heart failure: hypoventilation during sleep, reduced oxygen stores due to restricted lung volumes, sleep disordered breathing (SDB) and impaired gas exchange due to ventilation-perfusion mismatch. As previously, this section refers to patients who are either normoxic during the day, or have mild daytime hypoxaemia but do not fulfil LTOT criteria.

SDB in heart failure is due to central sleep apnoea (CSA) associated with Cheyne-Stokes respiration (CSR), often in combination with OSA. These frequently co-exist, and can be clinically difficult to differentiate. The presence of SDB is associated with atrial fibrillation and a worse New York Heart Association (NYHA) functional class. It is commoner in male patients, those over 60 years of age, and those with daytime hypocapnia $\left(\mathrm{PaCO}_{2}<5.06 \mathrm{kPa}, 38 \mathrm{~mm} \mathrm{Hg}\right) . \mathrm{SDB}$ in heart failure can have few symptoms and come to light following reports by carers, or presents with symptoms of disrupted sleep such as increased daytime sleepiness, poor subjective sleep quality, insomnia, inattention and poor concentration. Recurrent nocturnal desaturations can lead to paroxysmal nocturnal dyspnoea, morning headaches, nocturnal angina and arrhythmias. SDB is important in the context of heart failure as it can predict mortality and also contribute to disease progression (through intermittent hypoxaemia and arousals inducing adrenergic surges and negative intra-pleural pressure swings which increase left ventricular transmural pressure leading to an increase in afterload). Some international guidelines on heart failure management advocate screening for SDB in selected patients. Treatment approaches have been to maximise treatment for the underlying cardiac disorder, and to consider additional therapies which include NOT or ventilatory support such as continuous positive airways pressure (CPAP), adaptive servo-ventilation (ASV) or NIV.

There are significant limitations in the evidence of the impact of treatment of SDB on heart-related outcomes: most studies evaluate surrogate outcomes (blood pressure, cardiac function, catecholamines) rather than clinically important outcomes (health-related quality of life, hospitalisation and mortality). In addition, the literature evaluating NOT against modalities of ventilation is limited.

\section{Effect of NOT on SDB in severe cardiac disease}

Several studies examined the effects of low flow oxygen (2$4 \mathrm{~L} / \mathrm{min}$ delivered by nasal cannulae) on SDB in patients with moderate to severe heart failure. In two non-randomised studies, SDB was assessed using the Apnoea Hyponoea Index (AHI) and total sleep time as outcome measures, thus including both central and obstructive apnoeas. One of these trials reported findings on in-patients with severe heart failure and CSR on a transplant waiting list and showed that NOT led to a significant improvement in sleep quality after 1 night, which effect was sustained by use over 1 month (AHI reduced from $57 \pm 61$ events/h to $12 \pm 17$ events/h). ${ }^{66}$ In the other non-RCT of patients with moderate to severe heart failure (LVEF <45\%), NOT significantly reduced total AHI in $41 \%$ of patients (mainly reducing the CSA index) but did not affect total sleep time. ${ }^{67}$ Two RCTs of moderate to severe heart failure patients with CSR showed a reduction in CSR after 1 night of treatment with oxygen from $50.7 \pm 12 \%$ to $24.2 \pm 5.4 \%$ of total sleep time and after 4 weeks from 33.6 $\pm 7.4 \%$ to $10.7 \pm 3.9 \%$ of total sleep time, respectively. ${ }^{68}{ }^{69}$ In addition, Staniforth et $a l^{69}$ reported a reduction in CSAs from $18.4 \pm 4.1 / \mathrm{h}$ to $3.8 \pm 2.1 / \mathrm{h}$. Despite these improvements in sleep study parameters, no improvement in patient-reported symptom scores of sleep fragmentation were seen including the Epworth sleepiness scale and visual analogue scale (VAS), ${ }^{69}$ or the SF-A sleep questionnaire. ${ }^{70}$

\section{Effect of NOT on cardiac function in severe cardiac disease}

Studies examined cardiac function using transthoracic echocardiograms, assessment of NYHA functional class and plasma or urinary neuropeptide levels. No studies assessed the effects on nocturnal angina. No change in left ventricular function was seen following NOT. ${ }^{66} 6^{67} 7^{72}$ Despite no demonstrable improvement in echocardiogram parameters, one study reported a statistically significant improvement in NYHA functional class compared with an untreated control group after 52 weeks of NOT use. ${ }^{71}$ No studies demonstrated any effect on plasma or urinary neuropeptide levels. Two studies did not demonstrate any reduction in the frequency of ventricular arrhythmias during sleep. ${ }^{67} 73$

Effect of NOT on quality of life, activity and cognition in severe cardiac disease

Quality of life assessed using a disease-specific questionnaire in a 4 -week crossover study showed no improvement, ${ }^{69}$ whereas a case series also reporting after 4 weeks and using the Minnesota Living with Heart Failure (MLHF) Questionnaire did show 
improvement. ${ }^{74}$ Another study used the Dartmouth CO-OP Functional Health Assessment Charts which showed no improvement in daytime symptoms after 1 week. ${ }^{70}$

Exercise capacity was assessed in two case series of patients using NOT for 4 weeks ${ }^{74}$ and 3 months: ${ }^{72}$ both reported an improvement in the 6-minute walk test (6MWT). Activity assessed by the Specific Activity Scale showed significant improvement in a 52-week RCT. ${ }^{71}$

One study examined effects on cognitive function in detail in patients receiving NOT using a variety of measures. ${ }^{69}$ No improvements were seen after 4 weeks in this double-blind cross-over study. In contrast, Andreas et al used some similar measures which did demonstrate improvement after NOT. $^{70}$

\section{Effect of NOT on healthcare utilisation or mortality}

No studies evaluated the effects of NOT use on healthcare utilisation or mortality: most studies evaluate surrogate outcomes.

Evidence statements

- Treatment of heart failure patients who are symptomatic from SDB with NOT leads to a reduction in SDB. Evidence level 1-

- Treatment of symptomatic severe heart failure patients with NOT leads to modest improvement in exercise capacity. Evidence level 3

- Treatment of heart failure patients with NOT does not lead to improvement in quality of life, cognitive function, or cardiac function including ventricular arrhythmias. Evidence level $1+$

\section{Recommendation}

- NOT can be ordered for severe heart failure patients who do not fulfil indications for LTOT, and have evidence of SDB leading to daytime symptoms, after other causes of nocturnal desaturation have been excluded (eg, obesity hypoventilation or OSA) and heart failure treatment has been optimised. Treatment with modalities of ventilatory support should also be considered. (Grade B)

Good practice point

- If NOT is ordered for patients with severe heart failure, it should be ordered at a low flow rate of 1-2 L/min and response should be assessed by a reduction in symptoms of daytime sleepiness, and SDB indices as measured by an overnight oximetry study. A blood gas assessment should be undertaken to exclude worsening hypercapnia and respiratory acidosis. Treatment with modalities of ventilatory support should be considered for patients who are hypercapnic. $(\sqrt{ })$

\section{NOT in patients with other respiratory diseases and nocturnal desaturation}

Use of NOT in patients with CF

Patients with CF develop progressive airflow obstruction, ventilatory failure and nocturnal desaturations with sleep fragmentation (which may in addition result from cough). Development of nocturnal hypoxaemia and hypercapnia are known to be poor prognostic signs in patients with CF and use of NOT has been examined to see whether it will improve blood gas parameters and so improve prognosis. Four studies have examined the use of NOT in adult patients with CF, of which two studies were designed to evaluate the role of bi-level positive airway pressure (BiPAP) and used NOT, with and without air, in their control arm. All studies were single night studies of the varying modalities of NOT and between them examined effects on sleep quality, blood gas parameters and ventilation. No studies examined effects on pulmonary hypertension, quality of life, activity and cognition, or healthcare utilisation.

Twenty-eight patients with CF who received NOT were followed over 2 years. ${ }^{75}$ No statistically significant improvement in survival, lung or cardiac outcomes was seen, although school and work attendance had improved. However, actual hours of oxygen use were low. In a small RCT of 10 patients with mean $\mathrm{FEV}_{1}$ $<25 \%$ predicted (four with daytime hypercapnia), patients who were randomised to receive NOT over 2 nights rather than room air improved overnight oxygen saturation levels, but did not improve sleep parameters. ${ }^{76}$ Transcutaneous $\mathrm{PCO}_{2}$ rose in all stages of sleep, predominantly in REM sleep, but not to a level which was felt to be clinically significant. A small study of six subjects with mean $\mathrm{FEV}_{1}<29 \%$ predicted (two with daytime hypercapnia) reported results receiving room air, BiPAP or NOT over 3 nights in random order. ${ }^{77}$ NOT led to improved overnight oxygenation but no change in sleep quality. However, two patients developed symptomatic hypercapnia which was not seen with BiPAP and NOT given together, where there was substantial improvement in levels of hypercapnia. Another similar small study of 13 patients with mean $\mathrm{FEV}_{1}<32 \%$ predicted (six with daytime hypercapnia) showed a non-significant rise in transcutaneous $\mathrm{CO}_{2}$ with NOT which improved with BiPAP. ${ }^{78}$

Evidence statements

- Treatment of CF patients with NOT improves nocturnal oxygenation but there is no evidence of long-term benefit on survival. Evidence level 1+

- Treatment of CF patients with NOT does not improve sleep quality. Evidence level 1+

- Treatment of CF patients with NOT can cause hypercapnia, which can be improved with provision of NIV along with NOT. Evidence level 1+

Recommendation

- NOT should not be given to CF patients with nocturnal hypoxaemia alone who do not fulfil LTOT criteria. It can be considered in patients with evidence of established ventilatory failure, where it should be given with NIV support. (Grade B)

\section{Use of NOT in patients with ILD}

Patients with ILD have been found to develop progressive day and night-time hypoxaemia, sleep disruption and poor sleep quality. Evidence is limited in this area and no studies have examined the long-term use of nocturnal oxygen or its effects on mortality, pulmonary haemodynamics or healthcare utilisation in ILD. Only one study has prospectively examined the effect of NOT in patients with ILD compared with air. ${ }^{79}$ This was a 2-night study comparing room air with NOT titrated at $1-3 \mathrm{~L} / \mathrm{min}$ via nasal prongs to give an oxygen saturation reading of $>90 \%$. However, the study took place among long-term residents of Mexico city who were therefore acclimatised to living at altitude ('normal' control subjects had a mean $\mathrm{PaO}_{2}$ of $6.7 \mathrm{kPa}$ ). They found that NOT corrected nocturnal hypoxaemia, improved tachycardia and tachypnoea but that there was no change in sleep efficiency.

Evidence statements

- Treatment of ILD patients with nocturnal episodic hypoxaemia, but without established daytime blood gas abnormalities, with NOT improves nocturnal oxygenation, but there is no evidence of long-term benefit on survival. Evidence level 1+

- Treatment of ILD patients with NOT does not improve sleep quality. Evidence level 1+ 


\section{Recommendation}

- NOT should not be given to patients with ILD with nocturnal hypoxaemia alone, who do not fulfil LTOT criteria. (Grade B)

\section{Use of NOT in patients with neuromuscular weakness}

Patients with neuromuscular weakness can develop progressive weakness of all muscle groups including respiratory muscle weakness. If this occurs, they may develop nocturnal desaturation, particularly during REM sleep, prior to developing daytime type 2 respiratory failure. No studies have examined the long-term use of nocturnal oxygen or its effects on mortality, pulmonary haemodynamics or healthcare utilisation in neuromuscular weakness. Evidence comes from one study which examined the use of NOT in patients with Duchenne muscular dystrophy, who had normal daytime blood gases but evidence of episodic nocturnal hypoxaemia. ${ }^{80}$ No beneficial effect was found on sleep quality, but there was a significant worsening of the duration of hypopnoeas and central apnoeas. In addition, levels of hypercapnia were not monitored and the concern that NOT in the absence of NIV support may worsen ventilatory failure remains.

\section{Evidence statement}

- Treatment of patients with neuromuscular weakness and nocturnal episodic hypoxaemia with NOT, without established daytime blood gas abnormalities, does not improve sleep quality and worsens CSA. Evidence level 1+

\section{Recommendation}

- Patients with neuromuscular weakness affecting respiratory muscles should not have NOT alone ordered. It can be considered in patients with evidence of established ventilatory failure, where it should be given with NIV support. (Grade B)

\section{Use of NOT in patients with Cheyne-Stokes respiration, obesity} hypoventilation syndrome and overlap syndrome

Patients with OSA experience recurrent episodic desaturation throughout the night, which leads to sleep fragmentation, which usually manifests as daytime sleepiness. Treatment for moderate to severe OSA is with a combination of weight loss and CPAP treatment. Some obese patients may develop obesity hypoventilation syndrome (OHS) defined as obesity with body mass index (BMI) $>30 \mathrm{~kg} / \mathrm{m}^{2}$ and awake hypercapnia in the absence of other causes of hypoventilation. In these patients there can be sustained nocturnal hypoxaemia. Some patients can develop an 'overlap syndrome' in which there is a combination of OHS and OSA (often with underlying lung disease such as COPD) with worsening daytime ventilatory failure.

There are no trials of home oxygen therapy in the treatment of OHS or overlap syndrome. Oxygen has been used as an add-on therapy to NIV.

\section{Recommendation}

- Patients with OSA, OHS or overlap syndrome should not have NOT alone ordered. It can be considered in patients with evidence of established ventilatory failure, where it should be given with NIV support. (Grade D)

\section{AMBULATORY OXYGEN THERAPY}

AOT is defined as the use of supplemental oxygen during exercise and activities of daily living. ${ }^{81}$ In mobile patients who are not sufficiently hypoxaemic to qualify for LTOT but who desaturate on exercise, AOT has historically been used to optimise saturations and short-term exercise capacity. AOT is also often supplied to LTOT users, either to allow those who are mobile outdoors to optimise their exercise capacity and achieve their recommended hours per day usage, or to enable more immobile patients to leave the house in a wheelchair/scooter on occasion, for example for hospital appointments. In some patient groups such as those with CF, AOT may be used to maintain an exercise regime or to enable effective airways clearance.

\section{AOT in patients not eligible for LTOT}

There are a number of hypothetical benefits from the use of AOT in patients who are not hypoxaemic at rest but who desaturate on exercise, including increased oxygen transport, allowing greater utilisation of oxygen by exercising muscles, delayed onset of inspiratory muscle fatigue, reduction in symptoms of dyspnoea and improved right ventricular function.

Studies that have examined the use of AOT in non-LTOT users can be divided into those which have assessed the acute impact of AOT on exercise capacity during a single assessment, those studying the potential benefits of AOT during an exercise training programme, and those that have examined the potential longer term benefits of AOT on activity levels and quality of life.

\section{Use of AOT during exercise}

A Cochrane review of single assessment cross-over studies on the short-term impact of AOT versus placebo air on exercise capacity in moderate to severe COPD patients, reported that AOT significantly improved all outcomes of endurance exercise capacity (distance, time, number of steps) and that maximal exercise work rate also increased. ${ }^{82}$ Benefits in terms of reduced breathlessness, levels of oxygenation and minute ventilation at the time that the placebo test ended were also reported. However, the clinical significance of the size of improvement seen in these single assessment studies is unclear. ${ }^{83}$

The addition of supplemental oxygen during exercise training may allow patients who normally desaturate on exercise to tolerate higher levels of activity and therefore gain more from training. A meta- analysis included three RCTs examining the use of AOT during exercise training in COPD patients using comparable outcome measures. ${ }^{84}$ Although there were significant improvements in two parameters (constant power exercise time and constant power exercise end-of-test Borg score), there was no beneficial effect from oxygen-supplemented training in a number of other parameters including maximal exercise outcomes, functional exercise outcomes (6MWT), shuttle walk distance, health-related quality of life and oxygenation status. A recent single-blinded RCT examined use of AOT in a pulmonary rehabilitation programme in 51 'oxygen responders' who were selected based on whether they had $>10 \%$ improvement in exercise capacity when using AOT at baseline. ${ }^{85}$ Significant improvements in walking distance as measured by an endurance shuttle walking test pre- and post-course $(490 \mathrm{~m}, 95 \%$ CI 228 to $750 ; p \leq 0.001)$ were shown for those who had used AOT during pulmonary rehabilitation. This area has also been reviewed in the BTS Guideline on Pulmonary Rehabilitation in Adults 2013. ${ }^{86}$

In a study of CF patients with advanced lung disease and normal resting oxygen saturations, patients could exercise for longer periods using supplemental oxygen during graded exercise tests. ${ }^{87}$ In a Cochrane review of AOT in CF, six studies evaluated oxygen supplementation during exercise. Oxygenation improved, but mild hypercapnia resulted and participants receiving oxygen therapy were able to exercise for a significantly 
longer duration. ${ }^{24}$ Evidence for an effect of AOT on daily activity is lacking, but in clinical practice use of AOT to support exercise, physiotherapy and activities of daily living in patients with $\mathrm{CF}$ is commonplace.

\section{Long-term impact of AOT}

There are limited data on whether the symptomatic benefits outweigh the practical difficulties associated with using AOT in everyday life.

A large parallel double-blinded 12-week RCT randomised 143 COPD patients to use of AOT versus a control group using compressed air. ${ }^{88}$ There were no significant improvements in the AOT group in terms of dyspnoea, quality of life or functional capacity, although only 50 patients were shown to desaturate (defined in this case as $\mathrm{SpO}_{2}<88 \%$ ). Average cylinder usage in both groups was low at just $40 \mathrm{~min} /$ day, and $46 \%$ of the AOT group reported they would prefer to cease using oxygen therapy altogether at the end of the study. A number of smaller studies (ie, $n=20-45$ patients) have examined the shortterm benefits of AOT in either crossover or parallel blinded studies, lasting between 6 and 10 weeks. ${ }^{89-93}$ Modest statistically significant improvements were seen in exercise capacity ${ }^{93}$ and in health-related quality of life. ${ }^{89}$ However, the majority failed to show any sustained benefit from AOT in a number of variables, including dyspnoea, exercise capacity, St. George's Respiratory Questionnaire (SGRQ), health-related quality of life, activity levels, distance walked or time away from home.

\section{AOT in patients eligible for LTOT}

AOT is often ordered for LTOT patients, or those who require oxygen $24 \mathrm{~h}$ per day, to allow those who regularly mobilise outdoors to leave the house and maintain their oxygen saturations within desired levels. Conversely for patients who require LTOT or are dependent on oxygen $24 \mathrm{~h}$ per day, but are not able to mobilise outdoors, AOT may assist them to leave the house on an occasional basis, for example for hospital appointments.

Re-analysis of data from the NOTT study ${ }^{494}$ showed that in LTOT patients, AOT increased the chances of patients achieving the 15 oxygen hours per day threshold, which has been shown to confer survival benefits, a finding supported by other studies. ${ }^{95}$ However, AOT may prove burdensome for LTOT patients and not improve quality of life or exercise capacity. A 1 -year double-blinded crossover trial in 24 LTOT patients allocated them to one of: standard therapy of LTOT via an oxygen concentrator only, standard therapy plus AOT, or standard therapy plus ambulatory compressed air. Use of AOT did not improve any of the primary outcome measures, including quality of life, exercise tolerance or daily duration of oxygen use, and the trial was stopped prematurely after an interim analysis. ${ }^{96}$ However, patients were expected to collect their oxygen cylinders from the hospital themselves, which is likely to have had an impact on usage.

Some patients find the weight of standard cylinders prohibits use, and so lightweight cylinders may be considered. Use of lightweight cylinders for AOT in comparison with 'normal' weight cylinders had no impact on the hours of use or on activity levels, both of which were low at randomisation and throughout the study in a 6-month unblinded RCT of 17 LTOT patients. ${ }^{97}$ Poor compliance with AOT may result from lack of information provision, perceived unreliability of the delivery system, system weight, self-consciousness in public, and carer issues surrounding managing and using AOT equipment. ${ }^{98}$

\section{Assessment for AOT}

Oxygen saturation $\left(\mathrm{SpO}_{2}\right)$ measured from a finger probe or the earlobe, is frequently used in clinical practice during exercise to assess patients and their response to AOT. When oximetry in 20 COPD patients performing 6MWTs with AOT both noninvasively using ear-oximetry $\left(\mathrm{SpO}_{2}\right)$ and invasively using CO-oximetry $\left(\mathrm{SaO}_{2}\right)$ was compared, ${ }^{97}$ flow rates were incrementally increased until both $\mathrm{SaO}_{2}$ and $\mathrm{SpO}_{2}$ were $>90 \%$. Significant differences were noted between $\mathrm{SpO}_{2}$ and $\mathrm{SaO}_{2}$ readings, which would potentially have led to different flow rates being ordered in $50 \%$ of subjects. For patients with a high respiratory rate, for example those with CF or ILD, assessment using Venturi oxygen at a flow rate sufficient to exceed the patient's peak tidal (and exertional) inspiratory flow can offer advantages over oxygen therapy delivered by nasal cannulae. If total gas flow exceeds the patient's inspiratory flow rate, a Venturi mask will deliver an accurate oxygen concentration which may decrease the work of breathing and facilitate $\mathrm{CO}_{2}$ control. ${ }^{99}$ See the section on equipment for information on oxygen conservers.

\section{Evidence statements}

- AOT has been shown to improve survival in patients on LTOT by helping them to achieve $15 \mathrm{~h}$ per day usage. Evidence level $1++$

- AOT acutely increases exercise capacity in laboratory-based exercise tests in patients who are not eligible for LTOT but who desaturate during exercise. Evidence level 1+

- Long-term use of AOT has not been shown to confer any sustained benefits in dyspnoea, exercise capacity, functional capacity, time away from home or quality of life in patients who are not eligible for LTOT. Evidence level 1+

- AOT has not been shown to improve quality of life, exercise tolerance or oxygen usage in patients on LTOT. Evidence level 1-

- AOT leads to improvement in walking distance when given in a pulmonary rehabilitation programme setting to patients who have demonstrated a $>10 \%$ improvement in exercise capacity when using AOT at baseline assessment. Evidence level 1-

\section{Recommendations}

- AOT should not be routinely offered to patients who are not eligible for LTOT. (Grade B)

- AOT should not be routinely offered to patients already on LTOT. (Grade D)

- AOT assessment should only be offered to patients already on LTOT if they are mobile outdoors. (Grade A)

- AOT should be offered to patients for use during exercise in a pulmonary rehabilitation programme or during an exercise programme following a formal assessment demonstrating improvement in exercise endurance. (Grade B)

Good practice points

- Patients started on AOT should be reviewed regularly. If AOT was started during an exacerbation or when unwell, an initial review at 4-6 weeks to check it is still indicated is essential. $(\sqrt{ })$

- Home visits may be useful to identify problems with equipment or set-up. Further reviews should be carried out every 6 months when stable, or sooner if the patient's clinical status changes. $(\sqrt{ })$

- AOT therapy may offer patients with active lifestyles or active treatment regimens (eg, CF) additional benefits. All patients should be assessed for AOT in the context of their daily activity and therapies. $(\sqrt{ })$

- It is recognised that there may be some patients, for example with ILD and disabling breathlessness, who do not qualify 
for LTOT but who do desaturate on exercise, who may benefit from AOT. Once all other medical interventions have been optimised, these patients could be considered for AOT following formal assessment and AOT use could continue following demonstration of benefit and compliance. $(\sqrt{ })$

- Patients with high respiratory rates (common in CF and ILD) should receive AOT at a flow rate via a Venturi mask, which exceeds their peak tidal and exertional inspiratory flow, and be supplied with home oxygen equipment which is able to deliver the required high flow rates. $(\sqrt{ })$

- AOT may be offered to LTOT patients who could otherwise not achieve $15 \mathrm{~h}$ per day oxygen usage, or who are severely hypoxaemic and are too symptomatic to leave their house without supplemental oxygen but may need to do so, for example to attend GP or hospital appointments. Formal assessment is not required in these circumstances. $(\sqrt{ })$

For suggested patient selection criteria and an AOT assessment procedure for AOT during pulmonary rehabilitation, see appendix 1.

\section{PALLIATIVE OXYGEN THERAPY}

The term 'palliative oxygen therapy' (POT) refers to the use of oxygen to relieve the sensation of refractory persistent breathlessness in advanced disease or life-limiting illness irrespective of underlying pathology where all reversible causes have been or are being treated optimally.

Dyspnoea is common in patients with advanced life-limiting illness of all types. Breathlessness is a subjective sensation which arises from a complex interaction of physiological and psychological stimuli and processing. ${ }^{100}$ A number of small studies have demonstrated the benefit of non-pharmacological techniques such as breathing control/pacing, acupuncture ${ }^{101} 102$ or a hand-held fan, ${ }^{103}$ while the evidence supporting pharmacological management, principally opioids, is well established. ${ }^{104}$

This section discusses the role of home oxygen in the management of intractable breathlessness in patients with advanced cancer or end-stage cardiorespiratory disease. The evidence reviewed dates from subsequent studies since the publication of a report of the Expert Working Group of the Scientific Committee of the Association of Palliative Medicine on the use of oxygen in the palliation of breathlessness in 2004. ${ }^{105}$ However, as most participants in studies had an Eastern Cooperative Oncology Group (ECOG) performance status of 2 or 3 , this population might not be representative of the sickest patients in palliative care.

\section{Effects of POT in comparison with air}

The studies reviewed excluded patients with cognitive impairment ${ }^{106107}$ and those with a prognosis of $<1$ month. ${ }^{107}$ Oxygen was delivered by nasal cannula or mask, and flow rate varied from 2 to $5 \mathrm{~L} / \mathrm{min}$. Studies also varied regarding duration of oxygen therapy, ranging from $15 \mathrm{~min}^{106}$ and $60 \mathrm{~min}^{108}$ to 15 h. ${ }^{107}$ Oxygen was delivered at rest in two studies, ${ }^{106} 109$ on exertion in one ${ }^{108}$ and continuously for $15 \mathrm{~h}$ in another study. ${ }^{107}$ No studies looked at life expectancy, but one study reported quality of life. ${ }^{107}$

A double-blind crossover study randomised 51 patients with advanced cancer, 17 of whom were hypoxaemic $\left(\mathrm{SpO}_{2}<90 \%\right)$ to $15 \mathrm{~min}$ of either air or palliative oxygen. ${ }^{106}$ Patients (whether hypoxaemic at baseline or not) improved symptomatically with both air and oxygen, but there were no significant differences between the treatments. A systematic review and meta-analysis of the efficacy of palliative oxygen for relief of dyspnoea in hypoxaemic (mean $\mathrm{SpO}_{2}$ 88\%) or non-hypoxaemic cancer patients included 134 patients. ${ }^{108}$ Although palliative oxygen was administered in a variety of ways (nasal cannula or mask; rest or $6 \mathrm{MWT}$; flow rate $3-5 \mathrm{~L} / \mathrm{min}$ ), there was no improvement in dyspnoea. A double-blind RCT compared air with palliative oxygen $(2 \mathrm{~L} / \mathrm{min}$ for $15 \mathrm{~h}$ per day for 7 days from a concentrator) in 239 patients with cancer or end-stage cardiorespiratory disease. ${ }^{107}$ There was no statistically significant difference between the two groups in breathlessness (measured twice daily), frequency of side effects, or change in quality of life between groups. Finally, a cohort study failed to demonstrate any symptomatic benefit over 2 weeks of the provision of home palliative oxygen as measured by routine recording of breathlessness with each clinical encounter with a specialist community palliative care team. ${ }^{110}$

\section{Effects of POT in comparison with other therapies such as opiates, fan therapy and cognitive behaviour therapy}

There are no reported studies comparing POT with fan therapy, cognitive behavioural therapy or other techniques for symptomatic relief of breathlessness.

One study assessed the effects of oxygen and opioid treatment on ventilation and palliation of dyspnoea in hypoxaemic $\left(\mathrm{SpO}_{2}\right.$ $<90 \%)$ and non-hypoxaemic $\left(\mathrm{SpO}_{2} \geq 90 \%\right)$ palliative care patients (either opioid-naive or pre-treated with strong opioids) in a prospective non-randomised study. ${ }^{109}$ Whereas opioid administration resulted in a significant decrease in the intensity of dyspnoea in hypoxaemic or in non-hypoxaemic patients, nasal oxygen therapy did not. There was no significant correlation between intensity of dyspnoea and $\mathrm{SpO}_{2}$, and no significant difference between hypoxaemic or non-hypoxaemic patients with regard to transcutaneous $\mathrm{CO}_{2}$ increase or $\mathrm{SpO}_{2}$ decrease after administration of opioids.

\section{Evidence statements}

- Measurements of oxygenation do not correlate well with the subjective experience of dyspnoea in patients with cancer or end-stage cardiorespiratory disease. Evidence level 2+

- Hypoxaemic patients do not experience a significant difference in symptoms between air and POT despite having improved oxygen saturations when administered oxygen. Evidence level 2+

- Non-hypoxaemic patients or those with mild levels of hypoxaemia who would not normally qualify for LTOT do not experience symptomatic benefit with POT compared with air. Evidence level $1++$

- Opioids are significantly better than POT in reducing the intensity of dyspnoea in non-hypoxaemic or hypoxaemic patients. Evidence level 1+

\section{Recommendations}

- Patients with cancer or end-stage cardiorespiratory disease who are experiencing intractable breathlessness should not receive treatment with POT if they are non-hypoxaemic or have mild levels of hypoxaemia above current LTOT thresholds $\left(\mathrm{SpO}_{2} \geq 92 \%\right)$. (Grade A)

- Patients with cancer or end-stage cardiorespiratory disease who are experiencing intractable breathlessness should receive assessment for a trial of treatment with opiates from an appropriately trained healthcare professional. (Grade A)

- Patients with cancer or end-stage cardiorespiratory disease who are experiencing intractable breathlessness should receive assessment for a trial of treatment with nonpharmacological treatments including fan therapy, from an appropriately trained healthcare professional. (Grade D) 


\section{Good practice point}

- POT may on occasion be considered by specialist teams for patients with intractable breathlessness unresponsive to all other modalities of treatment. In those instances, individual formal assessment of the effect of palliative oxygen on reducing breathlessness and improving quality of life should be made. $(\sqrt{ })$

For suggested patient selection criteria and a protocol for POT assessment, see appendix 3 .

\section{SHORT BURST OXYGEN THERAPY}

SBOT is typically given to patients for the relief of breathlessness not relieved by any other treatments. It is used intermittently at home for short periods, for example 10-20 min at a time. Oxygen used in this way has traditionally been ordered for non-hypoxaemic patients and used for subjective relief of dyspnoea prior to exercise for oxygenation or after exercise for relief of dyspnoea and recovery from exertion.

\section{Use of SBOT in respiratory disease}

The studies reviewed were limited to patients with COPD and included normoxic and hypoxaemic patients with moderate to severe disease. Two studies examined oxygen delivery before and after exercise, while four studies administered oxygen after exercise. One study that examined the benefit of oxygen after exercise included patients given LTOT, ${ }^{111}$ however hypoxaemic and non-hypoxaemic patients were not analysed separately. There were no studies that specifically examined the benefits of SBOT ordered for hypoxaemic patients alone.

In order to examine the effect of supplemental oxygen before and after exercise in stable COPD patients with moderate to severe disease who demonstrated exercise desaturation, Nandi et al ${ }^{112}$ undertook two double-blind randomised studies. In the first study, 34 subjects received either cylinder air or oxygen $28 \%$ at a flow rate of $4 \mathrm{~L} / \mathrm{min}$ for $10 \mathrm{~min}$ before a $6 \mathrm{MWT}$. In the second study, 18 subjects received either cylinder air or cylinder oxygen for 5 min immediately after a $6 \mathrm{MWT}$. Those that took part in both studies did so on different days. Distance walked, oxygen saturations and breathlessness as measured by a VAS were recorded as was time to recovery. No difference was found in distance walked, subjective breathlessness or recovery time when oxygen was administered prior to exercise. Nor was there any significant difference in distance walked, recovery time or breathlessness when oxygen was administered following exercise. The authors concluded that no recommendation could be made to support a useful therapeutic role for SBOT.

Similarly, SBOT was not found to have any effect on performance when administered before and after exercise in 22 nonhypoxaemic COPD patients with moderate to severe disease. ${ }^{113}$ Subjects undertook four 6MWTs at each of two sessions. Cylinder air or oxygen was randomly administered prior to the first two walk tests and during recovery following the final two tests. The group found no significant difference in distance walked or breathlessness as measured by the Borg score for air and oxygen given prior to exercise and no significant difference in mean time to resting Borg score when oxygen was given after exercise.

Another study compared oxygen, air, fan and no treatment in 34 stable patients with moderate to severe COPD who were short of breath on minimal exertion and who were not hypoxaemic $\left(\mathrm{SaO}_{2} \leq 93 \%\right.$ at rest). ${ }^{114}$ Patients undertook an exercise step test on four occasions and after each test were given either oxygen $4 \mathrm{~L} / \mathrm{min}$ from a face mask, air from a face mask, air from a fan, or no intervention. Fourteen patients desaturated on exercise below 90\%. Oxygen therapy had no significant effect on Borg scores even for those patients who desaturated. Oxygen saturation rose more quickly and to a higher level when the oxygen mask was used compared with other treatments $(p<0.009)$, but this increase of $2 \%$ had no effect on subjective breathlessness as measured by the Borg score.

Patients were asked to choose whether they received treatment before or after exercise in a study that examined 22 stable COPD patients with moderate to severe disease. ${ }^{111}$ Subjects were studied at home undertaking an activity of choice (mean resting $\mathrm{SaO}_{2}$ 93.1\% (range 82-98\%)). All had domiciliary oxygen ordered for them and 50\% were on LTOT. In this double-blind study, cylinder air or oxygen was randomly administered after exercise. Interestingly, all subjects chose after exercise. The exercise was repeated after a rest period and the alternative treatment administered. There was no difference in recovery times with oxygen compared with air. Five patients were able to correctly identify oxygen from air on both occasions. This group had shorter subjective and objective recovery times when compared with the rest of the group, although this did not reach statistical significance.

A reduced recovery time as measured by a VAS was associated with oxygen use compared with compressed air or placebo in a study of 19 subjects with stable severe COPD (mean (SD) $\mathrm{PaO}_{2}$ $8.05(1.52) \mathrm{kPa}){ }^{115}$ Subjects undertook three step tests to maximal dyspnoea and then were administered either cylinder oxygen $67 \%$ via a mask, cylinder air at the same flow for $20 \mathrm{~min}$, or no mask in random order. The results were not found to be reproducible when the seven responders were re-tested after a time lapse of between 1 week and 1 month.

In a study undertaken by Stevenson and Calverley, 18 stable COPD patients were included, none of whom were hypoxaemic at rest, although six patients did desaturated on exercise (range 88-96\%). ${ }^{116}$ Patients attended on two occasions at least 1 week apart. At each visit, patients performed a maximal cardiorespiratory exercise test following which they randomly received either air or oxygen $\left(\mathrm{FiO}_{2} \mathrm{0.4}\right)$ in a single-blind crossover fashion. At one visit the subject remained instrumented during recovery, while at the other visit the mouthpiece and nose clips were replaced with a Venturi mask at a flow rate of $10 \mathrm{~L} / \mathrm{min}$. The results revealed that following exercise, administration of oxygen when compared with compressed air was associated with a reduced ventilatory effort and dynamic hyperinflation resolution was shorter; however, there was no reduction of breathlessness as measured by the Borg score at any time during recovery between oxygen and air inhalation, nor did oxygen influence the rate at which symptoms were resolved. The authors concluded that the routine use of oxygen to aid recovery of symptoms after exercise does not appear to be justified.

Healthcare utilisation and quality of life were measured in a randomised double-blind placebo-controlled trial in patients with moderate to severe disease following an admission to hospital with an exacerbation of COPD. ${ }^{117} \mathrm{~A}$ total of 78 non-hypoxaemic patients were recruited and were randomised to cylinder air, cylinder oxygen or usual care for 6 months following discharge from hospital. The subjects who were randomised to cylinder air or cylinder oxygen were instructed to use it at $2 \mathrm{~L} / \mathrm{min}$ via nasal cannulae as needed for relief of distressing or limiting breathlessness. Cylinder use was self-recorded in patient diaries. Healthcare utilisation was assessed by number of COPD-related readmissions and unscheduled emergency department or primary care visits. Quality of life was measured using the Chronic Respiratory Disease Questionnaire (CRQ), the Hospital Anxiety and Depression Scale (HAD) and the Medical Outcomes Study Short 
Form (SF-36). There was no significant difference between patient groups in any of the health-related quality of life measures apart from the emotions domain of the CRQ for the usual care group. Hospital readmission and healthcare utilisation were high. However, there was no significant difference between groups. Cylinder use was initially high but fell rapidly within weeks in both the cylinder air and oxygen groups. The authors conclude that these results do not offer any support for the use of SBOT on discharge from hospital following an acute exacerbation of COPD. Evidence statements

- SBOT does not improve exercise tolerance or reduce breathlessness when administered either before or following exercise to hypoxaemic or non-hypoxaemic patients with moderate to severe COPD. Evidence level $1++$

- SBOT does not improve health-related quality of life or reduce healthcare utilisation when ordered for patients following an acute exacerbation of COPD. Evidence level 1++ Recommendations

- SBOT should not be ordered for use prior to or following exercise in hypoxaemic or normoxic patients with COPD. (Grade A)

- SBOT should not be ordered on discharge from hospital for non-hypoxaemic patients with severe COPD. (Grade A)

\section{Use of SBOT in $\mathrm{CH}$}

$\mathrm{CH}$ pain is the most severe of the primary headache syndromes. It is characterised by periodic attacks of strictly unilateral pain associated with ipsilateral cranial autonomic symptoms. The majority of patients have episodic cluster headache (ECH), with cluster periods that typically occur in a circannual rhythm, while $10 \%$ have the chronic form $(\mathrm{CCH})$, with no significant remissions between cluster periods. High flow oxygen therapy is used to relieve pain and is delivered usually from static oxygen cylinders in the patient's home.

In a double-blind randomised, placebo-controlled cross-over trial, 109 adults were treated for $\mathrm{CH}$ attacks with either 100\% oxygen $(12 \mathrm{~L} / \mathrm{min})$ or inhaled air, given via a facial mask for $15 \mathrm{~min}$ at the start of an attack. Fifty-seven patients with ECH and 19 with $\mathrm{CCH}$ were available for the analysis. ${ }^{118}$ Oxygen was significantly superior to placebo in elimination of pain or provision of 'adequate pain relief' at $15 \mathrm{~min}$ in $78 \%$ of patients (vs $20 \%$ with air). In a case series of 52 randomly selected outpatients with either active $\mathrm{ECH}$ or $\mathrm{CCH}, 100 \%$ oxygen was administered through a facial mask at a rate of $7 \mathrm{~L} / \mathrm{min}$ for $15 \mathrm{~min}$ at the onset of each of 10 cluster attacks. ${ }^{119}$ Overall, $75 \%$ of patients obtained significant relief (defined as complete or almost complete reduction of pain in seven of 10 attacks within $15 \mathrm{~min}$ ) from cluster pain. These findings were supported by a double-blind crossover study of 19 patients where use of SBOT (6 L/min via non-rebreather face masks for $15 \mathrm{~min}$ ) produced significantly higher average relief scores for all oxygen-treated patients. ${ }^{120} \mathrm{~A}$ case report of three patients unresponsive to oxygen given at $7-10 \mathrm{~L} / \mathrm{min}$ reported complete or near complete alleviation of headache after $100 \%$ oxygen at a rate of $14-15$ $\mathrm{L} / \mathrm{min} .{ }^{121}$ Non-responders to SBOT have more often smoked in the past $(p=0.014)$, had longer $\mathrm{CH}$ attacks $(p=0.049)$, and reported more inter-ictal headache $(p=0.02)$ than responders. ${ }^{122}$

A single-blind crossover trial of 50 patients has compared sublingual ergotamine tartrate to SBOT $(100 \%$ oxygen via a face mask, at a rate of $7 \mathrm{~L} / \mathrm{min}$ for $15 \mathrm{~min}$ ) for symptomatic relief of cluster attacks showed no statistical difference between treatment groups. ${ }^{119}$
NICE (National Institute of Health and Care Excellence) guidelines on the diagnosis and management of headaches in young people and adults, published in September 2012, recommend oxygen and/or a subcutaneous or nasal triptan for the acute treatment of $\mathrm{CH}^{123}$ It is recommended that oxygen should be given at a flow rate of at least $12 \mathrm{~L} / \mathrm{min}$ with a non-rebreather mask and a reservoir bag arranged as home oxygen. (NICE refers to ambulatory oxygen in this context which differs from the definition of ambulatory oxygen used in this guideline).

Evidence statement

- SBOT delivering high flow oxygen $(12 \mathrm{~L} / \mathrm{min}$ via a nonrebreather mask) is an effective symptomatic treatment for acute $\mathrm{CH}$ attacks. Evidence level 1+

\section{Recommendation}

- SBOT delivering high flow oxygen therapy $(12 \mathrm{~L} / \mathrm{min}$ via a non-rebreather mask) should be offered to treat acute attacks of $\mathrm{CH}$. (Grade A)

\section{Good practice point}

- Appropriate equipment will need to be provided in order to ensure delivery of high flow rate oxygen at $12 \mathrm{~L} / \mathrm{min}$ for $\mathrm{CH}$ using a non-rebreather mask. Patients will usually have warning of a $\mathrm{CH}$ attack, and so provision should be made for urgent $4 \mathrm{~h}$ installation of home oxygen, if available, rather than a permanent home supply being provided. $(\sqrt{ })$

\section{EQUIPMENT FOR HOME OXYGEN THERAPY}

The equipment for home oxygen therapy can be divided into three categories: oxygen source (concentrators, cylinders and liquid oxygen), oxygen delivery (cannulae, masks, conservers and tracheal devices) and supplementary equipment (humidifiers and equipment to carry oxygen). Please see online supplementary appendix 12 for illustrations and further details of equipment types.

\section{Oxygen source: concentrators, cylinders and liquid oxygen - description and indications for use}

Home oxygen can be delivered from cylinders, concentrators or as liquid oxygen. Each of these oxygen sources can be static or portable, and the source selected is dependent upon the mobility and clinical circumstances of the patient, along with the costs of installation and supply as determined by the oxygen provider. There are few published studies comparing the different modes of oxygen source in different clinical situations. Some studies have compared similar devices, but many of these are now outdated and technology has superseded them.

\section{Concentrators}

The most common device for LTOT delivery is an oxygen concentrator which can either be fixed in a room in the house or is portable to go with the patient around the home, outside the home and in the workplace. An oxygen concentrator is an electrically driven device which takes room air and passes it through a filtering system, removing nitrogen, to supply an oxygenenriched gas mixture (usually $85-95 \%$ oxygen).

Performance of oxygen concentrators can vary depending on the technology used. ${ }^{124-130}$ The maximum oxygen concentration delivered by an oxygen concentrator is $96 \%,{ }^{124}$ but there can be a difference in performance between devices depending upon flow rate. In a study that assessed a number of oxygen concentrators, all concentrators were found to deliver sufficient oxygen to achieve target oxygen saturation levels above $92 \%$ at flow rates of $2 \mathrm{~L} / \mathrm{min}$, of $85-94 \%$ at $3 \mathrm{~L} / \mathrm{min}$ and of $69-85 \%$ at $4 \mathrm{~L} / \mathrm{min}$ depending upon the device. ${ }^{126}$ This can result in 
patients not receiving their oxygen as ordered. ${ }^{131}$ In another series of 2400 oxygen concentrator users, where the flow rate was $2 \mathrm{~L} / \mathrm{min}$ or less in $79 \%$ of users, the mean $\pm \mathrm{SD}$ oxygen saturation achieved was $92 \pm 6 \% .{ }^{127}$ It is current clinical practice to use a combination of two oxygen concentrators joined via a T-piece to deliver high flow rates, for example $12 \mathrm{~L} / \mathrm{min}$ when required, although there is no clinical trial evidence to support this practice and it is unknown whether the equipment used performs adequately in this way.

\section{Home concentrators}

Home concentrators will be installed and regularly maintained by oxygen provider companies. In order to reduce risk of falls from tripping over long lengths of tubing, they may be 'piped in' to the home with appropriate tubing to areas where the patient will use the oxygen (bedroom, living room). All concentrators should have fire breaks inserted into the tubing-one at the patient end and one at the machine end-to reduce the risk of potentially catastrophic fires (see the section on safety and home oxygen). Oxygen concentrators can deliver flow rates of up to $4 \mathrm{~L} / \mathrm{min}$, adjustable in $0.5 \mathrm{~L} / \mathrm{min}$ increments. Where low flow is needed, for example in paediatric, NIV use and oxygensensitive patients, flow metres that reduce flow can be added to the standard concentrator. High flow oxygen concentrators can deliver flow rates of $8 \mathrm{~L} / \mathrm{min}$. For very high flows, concentrators can be joined via a T-piece and each concentrator must be set to the same flow, for example $12 \mathrm{~L} / \mathrm{min}$ required would need two high flow concentrators both set at $6 \mathrm{~L} / \mathrm{min}$, although there is no research evidence to support their use in this way. This option may not be available and it is suggested that home oxygen teams check with their oxygen supplier. Concentrators are recommended for patients using oxygen for more than $1.4 \mathrm{~h}$ a day. ${ }^{132}$ Practical considerations for patients are the need to change filters weekly, regular servicing of the machine, the warm-up period of the machine and the noise of the device. A new development is a concentrator which can be used to refill small portable cylinders at home, known as a 'home fill' oxygen system.

\section{Transportable and portable concentrators}

Transportable concentrators are similar to home concentrators but smaller in size and more portable with a typical weight being $4.5-8.6 \mathrm{~kg}$. They come with batteries as well as a mains attachment, allowing use outside as well as inside the home. (Inside the home, a transportable concentrator can be used as a standard concentrator as well as fulfilling the patient's ambulatory needs.) The battery for use outside the home does limit the time they can be used without recharging and will depend on the flow rate and whether the pulsed mode is used. They can be used and charged in cars. Most are now approved for use on commercial aircraft, although patients are currently advised not to take their supplied equipment out of the country as it will not be supported by the oxygen supplier in the event of a malfunction when abroad. Current models are available that deliver up to $3 \mathrm{~L} / \mathrm{min}$ continuous oxygen and $6 \mathrm{~L} / \mathrm{min}$ pulsed oxygen, and come with a power adapter to plug into an electrical source, or a battery back-up.

Portable concentrators are somewhat lighter than transportable concentrators, with a typical weight being $3.3-4.5 \mathrm{~kg}$. The majority of portable oxygen concentrators provide pulsed oxygen only. Therefore, they are not suitable for use when sleeping. It should be noted that some portable concentrators have numerical settings, for example number 2 does not equate to $2 \mathrm{~L} / \mathrm{min}$, and some do not alarm when they malfunction.

\section{Cylinder oxygen}

A cylinder is a strengthened metal container containing compressed gas held under high pressure safely for use via its regulator (tap). Oxygen cylinders come in a range of sizes and hence capacity, ranging from small portable cylinders to large static cylinders (see online supplementary appendix 12), and are colour coded to distinguish them from other medical gases. Currently, oxygen cylinders are white with writing denoting the content down the side, and black with white shoulder: all medical oxygen cylinders will be white bodied by 2025. The flow rate can be fixed or variable depending on patient requirements. All systems containing compressed gases in the UK are subject to the Pressure Systems Safety Regulations 2000 (SI 2000 No 128), which are intended to prevent the risk of injury from pressurised systems.

Historically, static cylinders have usually been used to deliver short burst or palliative oxygen in the home but now find their main use as back-up cylinders if there is a power cut or concentrator failure or in the treatment of $\mathrm{CH}$ patients. Lightweight cylinders (example weight $8 \mathrm{~kg} / 3.6 \mathrm{lb}$ ) and standard ambulatory cylinders (example weight $3.2 \mathrm{~kg} / 7 \mathrm{lb}$ ) are available for ambulatory use.

\section{Liquid oxygen}

Liquid oxygen is oxygen that is cooled so that it condenses from a gas to a liquid which can be stored in insulated containers. Liquid oxygen is generally stored in large Dewar flasks with a decanting system to deliver it to smaller portable Dewar flasks. The length of time these can last will depend on the flow provided and the size of the Dewar flask. Users need to be trained to connect the two containers to reduce problems of gas leakage and also to prevent users received cold burns through inappropriate handing of the device. Choices between these devices should take account of individual patient's dexterity, visual acuity and strength. Liquid oxygen Dewar flasks can only be installed on a ground floor due to venting and safety considerations.

\section{Comparison of different oxygen sources in clinical trials}

The majority of clinical trials in this area focus on delivery of portable oxygen either to facilitate use of ambulatory oxygen or to use as a method of delivery of LTOT with home oxygen concentrators. There are six methods of delivering portable oxygen: liquid, home fill cylinder, portable cylinder, lightweight cylinder, portable and transportable oxygen concentrator.

\section{Use of portable oxygen to deliver ambulatory oxygen}

Several small RCTs have compared different modalities of portable oxygen ${ }^{97} 133134$ in short-term or exercise test-based studies. Comparison of standard portable cylinders with lightweight cylinders ${ }^{97}$ and safe-fill portable cylinders ${ }^{133}$ showed no difference in activity levels (which were low), oxygen saturation, Borg score or 6MWT between the different modalities. In a comparison of four different methods of supplying portable oxygen (liquid, home fill cylinder, portable concentrator and lightweight cylinder) in 44 patients with stable severe COPD, there were no differences between oxygen saturation, distance walked or time used. ${ }^{134}$ Cylinder oxygen was least favoured by patients and liquid oxygen was most favoured with the lowest long-term costs. Despite a lack of improvement in quality of life in this study, the patients using liquid oxygen in comparison with portable cylinders spent significantly longer outside the house and used their oxygen more. ${ }^{135}$ In another study of 
patients with severe COPD comparing continuous flow liquid oxygen with a portable concentrator, there was no significant difference in use or level of oxygenation. The flow rate of oxygen needed on ambulation was an average of three times higher than at rest. ${ }^{136}$

\section{Use of portable oxygen to deliver LTOT}

Small RCTs and an observational study have examined the use of portable oxygen in contributing to the delivery of LTOT. Portable devices compared with home-based LTOT alone improved oxygen usage. ${ }^{95}$ Use of liquid oxygen with or instead of a concentrator can increase daily use of oxygen ${ }^{137-139}$ and improve quality of life, ${ }^{137}$ but overall costs can be higher. ${ }^{137} 138$

\section{Static cylinder use}

A survey of patients using static oxygen cylinders at home found that most had a diagnosis of COPD and used oxygen regularly for short-term relief of breathlessness, with 58\% using their oxygen at least once a day. On average these patients used three cylinders each per month. ${ }^{140}$

Evidence statements

- Portable oxygen provides greater oxygen daily usage and improved quality of life than static concentrators alone. Evidence level 1-

- There is no conclusive difference in activity levels or utilisation between different methods of portable oxygen, but patient preference is generally for liquid oxygen. Evidence level 1-

- Lightweight cylinders do not improve walking distance or oxygen utilisation and may lead to increased costs. Evidence level 1-

- Oxygen concentrators are the most cost-effective way to deliver LTOT, but can have variable efficiency depending on flow rates, particularly above $4 \mathrm{~L} / \mathrm{min}$. Evidence level $2++$

\section{Recommendations}

- Oxygen concentrators should be used to deliver LTOT at flow rates of $4 \mathrm{~L} / \mathrm{min}$ or less. (Grade B)

- Portable oxygen should be delivered by whatever mode is best suited to the individual needs of the patient to increase the daily amount of oxygen used and activity levels in mobile patients. (Grade C)

Good practice point

- The type of portable device selected should balance patient factors with cost effectiveness, resources and safety. $(\sqrt{ })$

\section{Oxygen delivery: nasal cannulae and masks, oxygen-conserving devices and trans-tracheal devices- description and indications for use}

Methods of home oxygen delivery depend upon the patient's requirements and the setting for delivery of care. Interfaces used for home oxygen fall into two main categories: nasal cannulae and face masks using the Venturi system. Trans-tracheal delivery is rarely used but will be briefly described. In addition, oxygenconserving devices may be used to facilitate oxygen delivery. Most home oxygen tubing has a 'fire break' inserted at the patient end of the tubing just before the nasal cannula or mask: this is a thermal fuse which when triggered will stop the oxygen supply in the event of fire.

\section{Nasal cannulae and masks}

Nasal cannulae are the most common interface for oxygen delivery. This is largely the result of a compromise between patient comfort and tolerance when using oxygen for $15 \mathrm{~h} /$ day and the need for controlled oxygen concentration delivery. Nasal cannulae are usually lightweight, soft plastic/silicone tipped tubing that are dual-pronged and sit in the nostrils, held in position by looping the tubing over the pinna of the ears, and allow oxygen delivery continuously into the nose. The nasal cannula delivers a low flow of oxygen entrained in a larger volume of atmospheric air so that each litre per minute of oxygen flow adds about $3-4 \%$ to the inspired oxygen concentration. The respiratory rate as well as underlying disease process will determine the actual oxygen delivery. However, a small non-randomised trial showed that oxygen delivery with nasal cannulae can be very variable, with individual inspired oxygen concentrations varying between $24 \%$ and $35 \%$ with the same flow rate of $2 \mathrm{~L} / \mathrm{min} .{ }^{141}$ High flow nasal cannulae are used in critically unwell patients and not appropriate to the home oxygen population.

Oxygen masks are minimal volume, made of clear, soft plastic and held over the nose and mouth with elasticated straps for comfort. Venturi masks are designed to deliver accurate concentrations of oxygen when used with certain flow rates. They are favoured for delivery of controlled oxygen concentrations where this is clinically important, such as in patients with hypercapnic respiratory failure requiring LTOT. Other patient factors may be relevant such as confused or demented patients where flows might be altered in error.

Other interfaces such as the OxyArm have been developed allowing minimal head contact but no facial contact, and the potential for use in both nose and mouth breathers, and in patients with high respiratory rates. When used in stable COPD patients requiring LTOT over a 4-week period, nasal cannulae and OxyArm gave similar oxygen delivery, but fewer patients preferred the OxyArm due to dislodgement and reduced mobility. $^{142}$

\section{Oxygen-conserving devices}

Oxygen-conserving devices deliver oxygen during inspiration only and, by reducing oxygen wasted during expiration, enable cylinders to last longer compared to constant flow. This can reduce costs by reducing the number of home deliveries. Most oxygen delivery systems now have conservers fitted as standard. Each model of conserver will have very different specifications chosen by the manufacturers to suit the device and are not able to be changed by the users. This high degree of variability means that they are not truly comparable from one make or model to another. Reservoir cannulae are a form of oxygenconserving device but are rarely used in home oxygen services; information about them can be found in the BTS Emergency Oxygen guidelines. Historical studies performed prior to conservers becoming standard equipment have not been reviewed.

Most studies have agreed that conservers can reduce oxygen usage by as much as 50\%.126 143144 The demand oxygen delivery system produced only a small increase in walk distance without elevation of oxygen saturation, but was inferior to continuous flow oxygen in most of the measured variables when compared directly. ${ }^{145}$ However, it has been suggested that oxygen-conserving devices vary in their ability to maintain $\mathrm{SaO}_{2}$ levels during exercise $e^{146} 147$ and that some patients (particularly those who mouth breathe) may struggle to trigger them, and therefore patients should have ambulatory assessments before being issued with them. The evidence for the use of nocturnal oxygenation using a pulsed-dose oxygen-conserving device compared to continuous flow is limited. ${ }^{148}$ Continuous oxygen was compared with pulsed oxygen delivery at two different settings and showed no clinical difference. The evidence for the use of 
pulse dose conservers at night is at best very poor and requires more research.

\section{Trans-tracheal oxygen}

This form of oxygen can be used but rarely in the home setting and requires dedicated support from a trained team. Oxygen is delivered via a catheter inserted percutaneously between the second and third tracheal rings. By reducing anatomical dead space, it allows lower levels of oxygen to be required than nasal cannulae, and reduces the work of breathing. Serious complications can include catheter displacement, obstruction of the catheter by mucous, and infection.

\section{Evidence statements}

- Nasal cannulae can be used to deliver home oxygen at low flow rates and are acceptable to patients. Evidence level 4

- Nasal cannulae provide variable inspired concentrations of oxygen when used at the same flow rate in different patients. Evidence level 4

- Oxygen-conserving devices reduce total oxygen usage. Evidence level 1+

- Oxygen-conserving devices vary in their ability to maintain $\mathrm{SaO}_{2}$ levels during exercise, and some patients struggle to trigger them. Evidence level 1+

\section{Recommendations}

- Nasal cannulae should be considered as the first choice of delivery device for patients requiring home oxygen therapy. As an alternative, some patients may benefit from or prefer a Venturi mask system. (Grade D)

- Oxygen-conserving devices can be used in home oxygen patients requiring high flow rates to increase the time the cylinder will last. (Grade B)

\section{Good practice points}

- Venturi masks should be considered in patients in whom there are concerns about existing or developing hypercapnic respiratory failure, those with a high resting respiratory rate or those with cognitive problems. $(\sqrt{ })$

- Oxygen-conserving devices should be considered in patients who are active outside the home, following an ambulatory oxygen assessment. $(\sqrt{ })$

\section{Other equipment: trolleys and backpacks, humidifiers- description and indications for use}

Patient compliance with treatment is greatly improved with supplementary equipment which may help address practical issues around home oxygen provision.

\section{Humidification}

Oxygen is sometimes humidified in an attempt to prevent a drying effect of oxygen if delivered at high flow rates or in patients with excessive chest secretions such as those with CF or bronchiectasis. Systems are available for the humidification of supplemental oxygen by bubbling oxygen through sterile water. Whereas nebulised saline given in single doses can help airways clearance in the presence of thick secretions, there is no evidence to support the use of continuous humidification and the effect on patient comfort is negligible. ${ }^{147}{ }^{148}$ Some studies conclude the risks of infection contraindicate its use. ${ }^{149}{ }^{150}$ For patients with a tracheostomy tube, natural mechanisms to warm and moisturise inspired gases have been bypassed. It is therefore essential to humidify any supplemental oxygen being delivered to the tracheostomised patient to help maintain a patent tracheostomy tube, reduce the build-up of secretions within the inner tube or the tracheostomy itself, and minimise any subjective discomfort that the patient may experience. However, there are no trial data to evaluate this approach. More detail about the use of oxygen in tracheostomy patients is given in the BTS emergency oxygen guideline. $^{2}$

Evidence statement

- There is no evidence of patient benefit from use of humidified oxygen. Evidence level 3

\section{Recommendation}

- Humidification of home oxygen should not be ordered for non-tracheostomy patients. (Grade D)

Good practice point

- Patients receiving oxygen via a tracheostomy should receive humidified oxygen. $(\sqrt{ })$

\section{Carrying home oxygen: trolleys and backpacks}

Patients can benefit from the provision of trolleys, wheeled carts or backpacks to enable them to carry home oxygen equipment. This may be necessary because of the weight of the equipment when carried or to provide greater convenience. Less able patients find trolleys and wheeled carts easier to use than backpacks. ${ }^{149} 150$ Studies have shown that their use can improve patient quality of life, distance walked and symptoms during exercise in patients who are habitually mobile.

Evidence statement

- Trolleys or wheeled devices to enable patients to carry home oxygen can improve patient quality of life, distance walked and symptoms during exercise in patients who can walk more than $300 \mathrm{~m}$. Evidence level 1+

\section{Recommendation}

- Less able patients should be offered wheeled devices or backpacks if assessment shows they improve ambulation and quality of life. (Grade B)

Good practice point

- When being transported in cars, cylinders should be secured either with a seat belt, or in the foot-well or car boot, possibly using a cylinder box. Liquid oxygen should always be transported in an upright position. A warning triangle may be displayed and insurance companies should be informed. $(\sqrt{ })$

\section{SAFETY AND HOME OXYGEN THERAPY Smoking and home oxygen therapy}

There is increasing recognition of the significant risks of fire and personal injury associated with smoking and the use of home oxygen therapy. LTOT patients can be enabled to achieve smoking cessation, ${ }^{52}$ but despite these necessary interventions, many patients with respiratory disease, and especially COPD, continue to smoke. In addition, the clinician's assessment of smoking status relies mainly on patients' testimony and evidence has shown that this can be inaccurate. ${ }^{61}$ There have been no high quality trials to enable an objective assessment of the risks and benefits of the use of home oxygen in those who continue to smoke. However, there is emerging evidence from case reports of the risks of continued smoking and oxygen use. A study in four American states from 2000 to 2007 documented 38 fatalities associated with smoking and oxygen therapy, and 16 non-fatal injuries reported which included harm to two fire-fighters and one policeman. ${ }^{151}$ Of the fatalities, 34 (89\%) were using LTOT and smoking at the time the fire began, three were household members of smokers receiving LTOT, and one was a non-smoker with LTOT who was unintentionally ignited by a family member who was smoking. Two retrospective case series of patients admitted to burns units reported 
harm caused by smoking while using home oxygen. ${ }^{152} 153$ Of 27 patients over a 7-year period who were identified with burns directly attributed to home oxygen use, 24 were smoking while using oxygen, two were lighting pilot lights, and one was lighting his wife's cigarette. ${ }^{152}$ Of 21 patients in a 12 -year period who experienced partial thickness burn injuries, $57 \%$ sustained inhalation injury, five $(22 \%)$ required intubation and mechanical ventilation, and two died during hospitalisation. ${ }^{153}$ In addition, 86 home oxygen-related burn injuries were documented in a retrospective study designed to compare the outcome characteristics of patients admitted to a burns unit who had been intubated compared with those who had not been intubated. ${ }^{154}$ Lighting a cigarette was the cause of the majority of injuries (87\%), while exposure to other naked flame sources accounted for others (lighting a cooker $5 \%$, electrical spark 5\%, candles $2 \%$, and other open flames $1 \%$ ). There are an increasing number of anecdotal reports of e-cigarettes and chargers causing fire-related incidents if used in the vicinity of home oxygen.

No studies were identified that examined improvements in safety if smoking status in home oxygen patients was monitored either by urinary cotinine measurements or CO (carbon monoxide) monitoring compared to no monitoring.

\section{Role of risk assessments}

Recognition of the danger of fire and personal injury caused by smoking and home oxygen use has led to pragmatic approaches to individual assessment of risks on a case-by-case basis. A risk assessment may be conducted by the home oxygen assessment service and the fire and rescue service according to local protocols. Home oxygen suppliers carry out a formal risk assessment twice under the current UK National Framework Agreement: once at the time of taking the order and a field-based assessment at the patient's location when the order is delivered. Further risk assessment should then take place every 6 months thereafter. See appendix 4 for examples of risk assessment tools.

Some home oxygen services have adopted the practice of asking patients to sign a disclaimer acknowledging the risks of behaviours such as smoking near home oxygen.

\section{Responsibilities of the oxygen supplier}

Certain responsibilities around risk assessment are outlined in the National Framework Agreement for home oxygen services (December 2000, transitioned to NHS England 2013), which outlines the contractual obligations of home oxygen supply companies in England and Wales. In Scotland, a national home oxygen service was established by Health Facilities Scotland in 2012, and the single contracted supplier is also obliged to carry out a similar risk assessment prior to oxygen installation.

These risk assessment obligations for home oxygen suppliers in England are:

- A desk risk assessment should be conducted upon receipt of a home oxygen order to ensure that the oxygen equipment ordered matches the requirements and the equipment can be delivered safely.

- The supplier shall ensure that a field-based risk assessment is carried out at the time of installation to verify whether the requirement of the home oxygen order form (HOOF) can be supplied safely and in accordance with the requirements. See appendix 5 for details of HOOF forms.

- A field-based risk assessment must be conducted in each patient's primary and/or secondary location every 6 months after the initial field-based risk assessment to ensure that risk is monitored on an ongoing basis. The supplier shall file a copy of such field-based risk assessment and provide it to the clinical commissioning group home oxygen service lead where risk has been identified

- The field-based risk assessment will identify potential firerelated risk in the patient's home. During this assessment, the supplier shall check for the presence of an operational smoke detector or alarm. In the event that a smoke detector or fire alarm is not present, the supplier shall inform the local fire authorities of this fact, together with any specific fire risks that have been identified.

- The concentrator and any cylinders are positioned with sufficient ventilation and at a safe distance from any naked flame, cooking or heating appliance.

- Oxygen equipment should be placed in a position where it will not cause an obstruction to patients or family members, especially those who may have mobility or sight impairment.

- A fixed installation should be considered to fix tubing and reduce trip hazard.

- A second concentrator may be necessary in larger properties or if patients have difficulty using the stairs.

- Verbal and written information should be given to the patient or carer regarding the use of the equipment provided.

- The engineer should be satisfied that the patient can use the provided oxygen equipment safely.

- The oxygen concentrator must be checked regularly to ensure the filters are cleaned, the flow metres are accurate, and the concentrator delivers oxygen at the correct concentration.

- The oxygen contractor should inform the assessment service of any safety issues concerning the patient and the oxygen equipment and its use.

- The data collected by the oxygen contractor related to safety such as fire and accidents as a result of oxygen equipment and its use in the home, should be made available to oxygen assessment services.

\section{Role of the fire and rescue service}

Although this is not mandatory for the fire and rescue service, a community fire safety officer may visit to discuss fire safety, smoke alarms and safe exit routes in the event of a fire.

\section{Trips and falls}

Patients with home oxygen often have mobility or sight impairment, and equipment and tubing can jeopardise safety. No studies were found that examined the number of accidents that occurred as the result of home oxygen equipment or tubing.

\section{Evidence statements}

- Serious burns, inhalation injury or death can be caused by using oxygen while smoking or using oxygen near a naked flame. Evidence level 3

- Patients who are educated regarding the dangers of smoking and using oxygen are more likely to quit smoking. Evidence level $2+$

\section{Recommendations}

- Smoking cessation should be discussed and written education given to all patients prior to ordering home oxygen and at each subsequent review if the patient continues to smoke. (Grade C)

- Patients should be made aware in writing of the dangers of using home oxygen within the vicinity of any naked flame such as pilot lights, cookers, gas fires and candles. (Grade D)

- Patients and family members who continue to smoke in the presence of home oxygen should be warned of the associated dangers of smoking in the presence of oxygen. (Grade D) 


\section{Good practice points}

- Safety should be a factor when making decisions regarding ordering home oxygen. Education and written information should be provided to the patient and their family or carers regarding the safe use of oxygen and its equipment. $(\sqrt{ })$

- The risks of prescribing oxygen to active smokers should be considered on a case-by-case basis: this should include a home visit to assess the patient's home situation, attitude toward risks and smoking behaviour. Home oxygen assessment services may decide not to prescribe home oxygen to smokers if the risks are in their judgement too high. Particular consideration needs to be given to risks to children and risks to neighbours in multiple occupancy dwellings. A risk assessment tool should be used, and the health professional who is undertaking the risk assessment may need to visit the home in conjunction with the local fire service and/or the oxygen contractor. Where there is reasonable doubt, the therapy should not be prescribed. $(\sqrt{ })$

- Patients who continue to smoke or live with other household smokers should be informed that the order for home oxygen will be reviewed and evidence of increased risk may lead to withdrawal of home oxygen therapy. $(\sqrt{ })$

- Carbon monoxide monitoring and measuring urine cotinine may help identify those patients who continue to smoke. $(\sqrt{ })$

- Patients should be made aware that they should not use e-cigarettes and chargers within the vicinity of their home oxygen. $(\sqrt{ })$

- Oil-based emollients and petroleum jelly can support combustion in the presence of oxygen. Patients should be made aware that only water-based products should be used on the hands and face or inside the nose while using oxygen. $(\sqrt{ })$

- The oxygen supplier should be informed if the patient continues to smoke in order for the engineer to consider it in the home oxygen supplier risk assessment. $(\sqrt{ })$

- Patients and family or carers should be instructed not to remove the fire breaks or to change the flow rate on their oxygen equipment. Only oxygen tubing and connections supplied by the oxygen company should be used. $(\sqrt{ })$

- The local fire service should be made aware of patients who are using oxygen at home and especially those who continue to smoke in order for a home safety assessment to be carried out. $(\sqrt{ })$

- Patients and carers should be aware that tubing should be checked on a regular basis and repositioned as necessary to ensure safety by preventing trips and falls. $(\sqrt{ })$

\section{CONCLUSION}

This guideline has reviewed the indications for the ordering and provision of home oxygen. It has confirmed which patients will benefit from LTOT, how they should be assessed and monitored, focusing on difficult clinical situations such as hospital discharge and management of hypercapnia. It has outlined recommended flow rates and duration of use, along with a review of all modalities of equipment used to deliver home oxygen. It has given recommendations for use of NOT in patients with advanced cardiac disease who are symptomatic from SDB and for use of SBOT in acute $\mathrm{CH}$ alone. It has recommended use of AOT is limited to patients with evidence of improvement of exercise tolerance when using AOT as part of a pulmonary rehabilitation programme. Finally, it has considered in detail the safety aspects of home oxygen delivery, particularly in the challenging area of risk assessment in continuing smokers.

\section{Author affiliations}

${ }^{1}$ Oxford Centre of Respiratory Medicine, Oxford University Hospitals NHS Trust, Oxford, UK

${ }^{2}$ Hywel Dda University Health Board, Prince Philip Hospital, Llanelli, UK

${ }^{3}$ Portsmouth NHS Trust, Portsmouth, UK

${ }^{4}$ Lung Function and Sleep, Queen Elizabeth Hospital Birmingham, Birmingham, UK

${ }^{5}$ Staffordshire and Stoke-on-Trent Partnership Trust, Staffordshire, UK

${ }^{6}$ Mundesley Medical Centre, Mundesley, UK

${ }^{7}$ Improvement Academy (Y\&H AHSN) Bradford Teaching Hospitals NHS FT, Wakefield, UK

${ }^{8}$ Royal Brompton Hospital, London, UK

${ }^{9}$ Respiratory Care Team, Virgin Care, Surrey, UK

${ }^{10}$ University of Edinburgh, Edinburgh, UK

${ }^{11}$ Department of Physiotherapy, Guy's and St Thomas' NHS Foundation Trust, St Thomas' Hospital, London, UK

${ }^{12}$ Aintree University Hospital, Liverpool, UK

${ }^{13}$ Respiratory Department, Royal United Hospital, Bath, UK

${ }^{14}$ Northern General Hospital, Sheffield, UK

${ }^{15} \mathrm{Clinical}$ and Experimental Medicine, Faculty of Medicine, University of Southampton, Southampton, UK

Contributors $\mathrm{MH}$, as chair of the guideline development group oversaw the development of the guideline, adhering to British Thoracic Society guideline methodology, and is responsible for the final guideline document. JA, SB, BC, $A E$, $D F, A G, S H, V K, W M, L M, K P, J S, V V$, TW, all members of the British Thoracic Society Home Oxygen Guideline Development Group, were responsible for development of the scope of the guideline, review of evidence, drafting text and recommendations.

Competing interests All guideline group members completed the BTS declaration of interest form in line with BTS policy, and copies of all declarations are available on the BTS website or on request from BTS Head Office.

Provenance and peer review Not commissioned; internally peer reviewed.

\section{REFERENCES}

1 Balfour-Lynn IM, Field DJ, Gringras P, et al. BTS guidelines for home oxygen in children. Thorax 2009;64(Suppl 2):ii1-26.

2 O'Driscoll BR, Howard LS, Davison AG, et al. BTS guideline for emergency oxygen use in adult patients. Thorax 2008;63(Suppl 6):vi1-68.

3 Ahmedzai S, Balfour-Lynn IM, Bewick T, et al. Managing passengers with stable respiratory disease planning air travel: British Thoracic Society recommendations. Thorax 2011;66(Suppl 1):i1-30.

4 [No authors listed]. Continuous or nocturnal oxygen therapy in hypoxemic chronic obstructive lung disease: a clinical trial. Nocturnal Oxygen Therapy Trial Group. Ann Int Med 1980:93:391-8.

5 [No authors listed]. Long term domiciliary oxygen therapy in chronic hypoxic cor pulmonale complicating chronic bronchitis and emphysema. Report of the Medical Research Council Working Party. Lancet 1981;1:681-6.

6 Cooper CB, Waterhouse J, Howard P. Twelve year clinical study of patients with hypoxic cor pulmonale given long term domiciliary oxygen therapy. Thorax 1987:42:105-10.

7 Strom K. Survival of patients with chronic obstructive pulmonary disease receiving long-term domiciliary oxygen therapy. Am Rev Respir Dis 1993;147:585-91.

8 Gulbas G, Gunen H, In E, et al. Long-term follow-up of chronic obstructive pulmonary disease patients on long-term oxygen treatment. Int I Clin Pract 2012;66:152-7.

9 Górecka D, Gorzelak K, Sliwiński P, et al. Effect of long-term oxygen therapy on survival in patients with chronic obstructive pulmonary disease with moderate hypoxaemia. Thorax 1997;52:674-9.

10 Machado M-CL, Krishnan JA, Buist SA, et al. Sex differences in survival of oxygen-dependent patients with chronic obstructive pulmonary disease. Am J Respir Crit Care Med 2006;174:524-9.

11 Zielinski J, MacNee W, Wedzicha J, et al. Causes of death in patients with COPD and chronic respiratory failure. Monaldi Arch Chest Dis 1997;52:43-7.

12 Chailleux E, Laaban J-P, Veale D. Prognostic value of nutritional depletion in patients with COPD treated by long-term oxygen therapy: data from the ANTADIR observatory. Chest 2003;123:1460-6.

13 Fleetham JA, Bradley CA, Kryger MH, et al. The effect of low flow oxygen therapy on the chemical control of ventilation in patients with hypoxemic COPD. Am Rev Respir Dis 1980;122:833-40.

14 Timms RM, Khaja FU, Williams GW. Hemodynamic response to oxygen therapy in chronic obstructive pulmonary disease. Ann Int Med 1985;102:29-36.

15 MacNee W, Morgan AD, Wathen CG, et al. Right ventricular performance during exercise in chronic obstructive pulmonary disease. The effects of oxygen. Respiration 1985;48:206-15. 
16 Calverley PM, Brezinova V, Douglas NJ, et al. The effect of oxygenation on sleep quality in chronic bronchitis and emphysema. Am Rev Respir Dis 1982;126:206-10.

17 Okubadejo AA, Paul EA, Jones PW, et al. Does long-term oxygen therapy affect quality of life in patients with chronic obstructive pulmonary disease and severe hypoxaemia? Eur Respir J 1996;9:2335-9.

18 Heaton RK, Grant I, McSweeny AJ, et al. Psychologic effects of continuous and nocturnal oxygen therapy in hypoxemic chronic obstructive pulmonary disease. Arch Intern Med 1983;143:1941-7.

19 Borak J, Sliwinski P, Tobiasz M, et al. Psychological status of COPD patients before and after one year of long-term oxygen therapy. Monaldi Arch Chest Dis 1996:51:7-11.

20 Garcia-Aymerich J, Monso E, Marrades RM, et al. Risk factors for hospitalization for a chronic obstructive pulmonary disease exacerbation. EFRAM study. Am J Respir Crit Care Med 2001;164:1002-7.

21 Ringbaek TJ, Viskum K, Lange P. Does long-term oxygen therapy reduce hospitalisation in hypoxaemic chronic obstructive pulmonary disease? Eur Respir $J$ 2002;20:38-42.

22 Bratel $T$, Ljungman $S$, Runold $M$, et al. Renal function in hypoxaemic chronic obstructive pulmonary disease: effects of long-term oxygen treatment. Respir Med 2003:97:308-16.

23 Chambellan A, Chailleux E, Similowski T. Prognostic value of the hematocrit in patients with severe COPD receiving long-term oxygen therapy. Chest 2005; 128:1201-8

24 Elphick Heather E, Mallory G. Oxygen therapy for cystic fibrosis. Cochrane Database Syst Rev 2013;(7):CD003884.

25 Hilberink SR, Jacobs JE, van Opstal S, et al. Validation of smoking cessation self-reported by patients with chronic obstructive pulmonary disease. Int I Gen Med 2011:4:85-90.

26 Calverley PM, Leggett RJ, McElderry L, et al. Cigarette smoking and secondary polycythemia in hypoxic cor pulmonale. Am Rev Respir Dis 1982;125:507-10.

27 NHS Medical Directorate. Service Specification: Home oxygen assessment and review service. 2012

28 Roberts CM, Bugler JR, Melchor R, et al. Value of pulse oximetry in screening for long-term oxygen therapy requirement. Eur Respir J 1993;6:559-62.

29 Roberts CM, Franklin J, O'Neill A, et al. Screening patients in general practice with COPD for long-term domiciliary oxygen requirement using pulse oximetry. Respir Med 1998;92:1265-8.

30 Carlin BW, Clausen JL, Ries AL. The use of cutaneous oximetry in the prescription of long-term oxygen therapy. Chest 1988;94:239-41.

31 Guyatt GH, Nonoyama M, Lacchetti C, et al. A randomized trial of strategies for assessing eligibility for long-term domiciliary oxygen therapy. Am J Respir Crit Care Med 2005; 172:573-80.

32 Timms RM, Kvale PA, Anthonisen NR, et al. Selection of patients with chronic obstructive pulmonary disease for long-term oxygen therapy. JAMA 1981;245:2514-15.

33 Chaney JC, Jones K, Grathwohl K, et al. Implementation of an oxygen therapy clinic to manage users of long-term oxygen therapy. Chest 2002;122:1661-7.

34 Oba Y, Salzman GA, Willsie SK. Reevaluation of continuous oxygen therapy after initial prescription in patients with chronic obstructive pulmonary disease. Respir Care 2000;45:401-6.

35 Eaton T, Rudkin S, Garrett JE. The clinical utility of arterialized earlobe capillary blood in the assessment of patients for long-term oxygen therapy. Respir Med 2001;95:655-60.

36 Levi-Valensi P, Weitzenblum E, Pedinielli JL, et al. Three-month follow-up of arterial blood gas determinations in candidates for long-term oxygen therapy. A multicentric study. Am Rev Respir Dis 1986;133:547-51.

37 Eaton T, Young P, Fergusson W, et al. A double-blind randomised controlled study of portable oxygen in patients with COPD and exertional desaturation. Am J Respir Crit Care Med 2001;163(5 Suppl):A499.

38 Munoz X, Torres F, Sampol G, et al. Accuracy and reliability of pulse oximetry at different arterial carbon dioxide pressure levels. Eur Respir J 2008;32:1053-9.

39 Hughes JM. Blood gas estimations from arterialized capillary blood versus arterial puncture: are they different? Eur Respir J 1996;9:184-5.

40 Zavorsky GS, Cao J, Mayo NE, et al. Arterial versus capillary blood gases: a meta-analysis. Respir Physiol Neurobiol 2007;155:268-79.

41 Pitkin $A D$, Roberts $C M$, Wedzicha JA. Arterialised earlobe blood gas analysis: an underused technique. Thorax 1994;49:364-6.

42 Schafroth Torok S, Leuppi JD, Baty F, et al. Combined oximetry-cutaneous capnography in patients assessed for long-term oxygen therapy. Chest 2008;133:1421-5.

43 Chiang LL, Hung TC, Ho SC, et al. Respiratory response to carbon dioxide stimulation during low flow supplemental oxygen therapy in chronic obstructive pulmonary disease. J Formos Med Assoc 2002;101:607-15.

44 Pilling J, Cutaia M. Ambulatory oximetry monitoring in patients with severe COPD: a preliminary study. Chest 1999;116:314-21.

45 Morrison DA, Stovall JR. Increased exercise capacity in hypoxemic patients after long-term oxygen therapy. Chest 1992;102:542-50.
46 Sliwinski P, Lagosz M, Gorecka D, et al. The adequacy of oxygenation in COPD patients undergoing long-term oxygen therapy assessed by pulse oximetry at home. Eur Respir J 1994;7:274-8.

47 Abdulla J, Godtfredsen N, Pisinger C, et al. Adequacy of oxygenation in a group of Danish patients with COPD on long-term oxygen therapy. Monaldi Arch Chest Dis 2000:55:279-82.

48 Zhu Z, Barnette RK, Fussell KM, et al. Continuous oxygen monitoring-a better way to prescribe long-term oxygen therapy. Respir Med 2005;99:1386-92.

49 Morrison D, Skwarski KM, MacNee W. The adequacy of oxygenation in patients with hypoxic chronic obstructive pulmonary disease treated with long-term domiciliary oxygen. Respir Med 1997;91:287-91.

50 Nisbet $M$, Eaton $T$, Lewis $C$, et al. Overnight prescription of oxygen in long term oxygen therapy: time to reconsider the guidelines? Thorax 2006;61:779-82.

51 Plywaczewski R, Sliwinski P, Nowinski $A$, et al. Incidence of nocturnal desaturation while breathing oxygen in COPD patients undergoing long-term oxygen therapy. Chest 2000;117:679-83.

52 Peckham DG, McGibbon K, Tonkinson J, et al. Improvement in patient compliance with long-term oxygen therapy following formal assessment with training. Respir Med 1998;92:1203-6.

53 Pepin JL, Barjhoux CE, Deschaux C, et al. Long-term oxygen therapy at home. Compliance with medical prescription and effective use of therapy. ANTADIR Working Group on Oxygen Therapy. Association Nationale de Traitement a Domicile des Insuffisants Respiratories. Chest 1996;109:1144-50.

54 Eaton TE, Grey C, Garrett JE. An evaluation of short-term oxygen therapy: the prescription of oxygen to patients with chronic lung disease hypoxic at discharge from hospital. Respir Med 2001;95:582-7.

55 Cottrell JJ, Openbrier D, Lave JR, et al. Home oxygen therapy. A comparison of 2- vs 6-month patient reevaluation. Chest 1995;107:358-61.

56 Granados A, Escarrabill J, Borras JM, et al. The importance of process variables analysis in the assessment of long-term oxygen therapy by concentrator. Respir Med 1997;91:89-93.

57 Godoy I, Tanni SE, Hernandez C, et al. The importance of knowing the home conditions of patients receiving long-term oxygen therapy. Int J Chron Obstruct Pulmon Dis 2012;7:421-5.

58 Rizzi M, Grassi M, Pecis $M$, et al. A specific home care program improves the survival of patients with chronic obstructive pulmonary disease receiving long term oxygen therapy. Arch Phys Med Rehabil 2009;90:395-401.

59 Farrero E, Escarrabill J, Prats E, et al. Impact of a hospital-based home-care program on the management of COPD patients receiving long-term oxygen therapy. Chest 2001;119:364-9.

60 Goldbart J, Yohannes AM, Woolrych R, et al. 'It is not going to change his life but it has picked him up': a qualitative study of perspectives on long term oxygen therapy for people with chronic obstructive pulmonary disease. Health Qual Life Outcomes 2013:11:124.

61 Restrick LJ, Paul EA, Braid GM, et al. Assessment and follow up of patients prescribed long term oxygen treatment. Thorax 1993;48:708-13.

62 Chaouat $A$, Weitzenblum $E$, Kessler $R$, et al. A randomized trial of nocturnal oxygen therapy in chronic obstructive pulmonary disease patients. Eur Respir $J$ 1999;14:1002-8.

63 McKeon JL, Murree-Allen K, Saunders NA. Supplemental oxygen and quality of sleep in patients with chronic obstructive lung disease. Thorax 1989;44:184-8.

64 Fletcher EC, Donner CF, Midgren B, et al. Survival in COPD patients with a daytime $\mathrm{PaO} 2$ greater than $60 \mathrm{~mm} \mathrm{Hg}$ with and without nocturnal oxyhemoglobin desaturation. Chest 1992;101:649-55.

65 Fletcher EC, Luckett RA, Goodnight-White S, et al. A double-blind trial of nocturnal supplemental oxygen for sleep desaturation in patients with chronic obstructive pulmonary disease and a daytime PaO2 above $60 \mathrm{~mm} \mathrm{Hg}$. Am Rev Respir Dis 1992;145:1070-6.

66 Krachman SL, Nugent T, Crocetti J, et al. Effects of oxygen therapy on left ventricular function in patients with Cheyne-Stokes respiration and congestive heart failure. J Clin Sleep Med 2005;1:271-6.

67 Javaheri S, Ahmed M, Parker TJ, et al. Effects of nasal 02 on sleep-related disordered breathing in ambulatory patients with stable heart failure. Sleep 1999:22:1101-6.

68 Hanly PJ, Millar TW, Steljes DG, et al. The effect of oxygen on respiration and sleep in patients with congestive heart failure. Ann Int Med 1989;111:777-82.

69 Staniforth AD, Kinnear WJM, Starling R, et al. Effect of oxygen on sleep quality, cognitive function and sympathetic activity in patients with chronic heart failure and Cheyne-Stokes respiration. Eur Heart J 1998;19:922-8.

70 Andreas S, Clemens C, Sandholzer H, et al. Improvement of exercise capacity with treatment of Cheyne-Stokes respiration in patients with congestive heart failure. J Am Coll Cardiol 1996:27:1486-90.

71 Sasayama S, Izumi T, Matsuzaki M, et al. Improvement of quality of life with nocturnal oxygen therapy in heart failure patients with central sleep apnea. Circ J 2009;73:1255-62

72 Brostrom A, Hubbert L, Jakobsson $\mathrm{P}$, et al. Effects of long-term nocturnal oxygen treatment in patients with severe heart failure. J Cardiovasc Nurs 2005;20:385-96. 
73 Suzuki J, Ishihara T, Sakurai K, et al. Oxygen therapy prevents ventricular arrhythmias in patients with congestive heart failure and sleep apnea. Circ J 2006;70:1142-7.

74 Paul B, Joseph M, De Pasquale CG. Domiciliary oxygen therapy improves sub-maximal exercise capacity and quality of life in chronic heart failure. Heart Lung Circ 2008;17:220-3.

75 Zinman R, Corey M, Coates AL, et al. Nocturnal home oxygen in the treatment of hypoxemic cystic fibrosis patients. J Pediatr 1989;114:368-77.

76 Spier S, Rivlin J, Hughes D, et al. The effect of oxygen on sleep, blood gases, and ventilation in cystic fibrosis. Am Rev Respir Dis 1984;129:712-18.

77 Gozal D. Nocturnal ventilatory support in patients with cystic fibrosis: comparison with supplemental oxygen. Eur Respir J 1997;10:1999-2003.

78 Milross MA, Piper AJ, Norman M, et al. Low-flow oxygen and bilevel ventilatory support: Effects on ventilation during sleep in cystic fibrosis. Am J Respir Crit Care Med 2001;163:129-34.

79 Vazquez JC, Perez-Padilla R. Effect of oxygen on sleep and breathing in patients with interstitial lung disease at moderate altitude. Respiration 2001;68:584-9.

80 Smith PE, Edwards RH, Calverley PM. Oxygen treatment of sleep hypoxaemia in Duchenne muscular dystrophy. Thorax 1989:44:997-1001.

81 Wedzicha JA. Domiciliary oxygen therapy services: clinical guidelines and advice for prescribers. Summary of a report of the Royal College of Physicians. J $R$ Coll Physicians Lond 1999;33:445-7.

82 Bradley JM, O'Neill BM. Short-term ambulatory oxygen for chronic obstructive pulmonary disease. Cochrane Database Syst Rev 2005;(4):CD004356.

83 Bradley JM, Lasserson T, Elborn S, et al. A systematic review of randomized controlled trials examining the short-term benefit of ambulatory oxygen in COPD (Brief record). Chest 2007;131:278-85.

84 Nonoyama M, Brooks D, Lacasse $Y$, et al. Oxygen therapy during exercise training in chronic obstructive pulmonary disease. Cochrane Database Syst Rev 2007;(2): CD005372.

85 Dyer F, Callaghan J, Cheema K, et al. Ambulatory oxygen improves the effectiveness of pulmonary rehabilitation in selected patients with chronic obstructive pulmonary disease. Chron Respir Dis 2012;9:83-91.

86 Bolton CE, Bevan-Smith EF, Blakey JD, et al. British Thoracic Society guideline on pulmonary rehabilitation in adults. Thorax 2013:68(Suppl 2):ii1-30.

87 Marcus CL, Bader D, Stabile MW, et al. Supplemental oxygen and exercise performance in patients with cystic fibrosis with severe pulmonary disease. Chest 1992;101:52-7.

88 Moore RP, Berlowitz DJ, Denehy L, et al. A randomised trial of domiciliary, ambulatory oxygen in patients with COPD and dyspnoea but without resting hypoxaemia. Thorax 2011;66:32-7.

89 Eaton T, Garrett JE, Young P, et al. Ambulatory oxygen improves quality of life of COPD patients: a randomised controlled study. Eur Respir J 2002;20:306-12

90 Nonoyama ML, Brooks D, Guyatt GH, et al. Effect of oxygen on health quality of life in patients with chronic obstructive pulmonary disease with transient exertional hypoxemia. Am J Respir Crit Care Med 2007;176:343-9.

91 Sandland CJ, Morgan MD, Singh SJ. Patterns of domestic activity and ambulatory oxygen usage in COPD. Chest 2008;134:753-60.

92 Ringbaek T, Martinez G, Lange P. The long-term effect of ambulatory oxygen in normoxaemic COPD patients: a randomised study. Chron Respir Dis 2013;10:77-84. http://onlinelibrary.wiley.com/o/cochrane/clcentral/articles/157/ CN-00861157/frame.htm

93 McDonald CF, Blyth CM, Lazarus MD, et al. Exertional oxygen of limited benefit in patients with chronic obstructive pulmonary disease and mild hypoxemia. Am J Respir Crit Care Med 1995;152(5 Pt 1):1616-19.

94 Petty TL, Bliss PL. Ambulatory oxygen therapy, exercise, and survival with advanced chronic obstructive pulmonary disease (the Nocturnal Oxygen Therapy Trial revisited). Respir Care 2000:45:204-11; discussion 11-3.

95 Vergeret J, Brambilla C, Mounier L. Portable oxygen therapy: use and benefit in hypoxaemic COPD patients on long-term oxygen therapy. Eur Respir $J$ $1989 ; 2: 20-5$.

96 Lacasse $Y$, Lecours $R$, Pelletier $C$, et al. Randomised trial of ambulatory oxygen in oxygen-dependent COPD. Eur Respir J 2005;25:1032-8.

97 Casaburi R, Porszasz J, Hecht A, et al. Influence of lightweight ambulatory oxygen on oxygen use and activity patterns of COPD patients receiving long-term oxygen therapy. COPD 2012;9:3-11.

98 Arnold E, Bruton A, Donovan-Hall M, et al. Ambulatory oxygen: why do COPD patients not use their portable systems as prescribed? A qualitative study. $B M C$ Pulm Med 2011:11:9.

99 Hill SL, Barnes PK, Hollway T, et al. Fixed performance oxygen masks: an evaluation. Br Med J (Clin Res Ed) 1984:288:1261-3.

100 Ripamonti C, Bruera E. Dyspnea: pathophysiology and assessment. J Pain Symptom Manage 1997;13:220-32.

101 Corner J, Plant H, A'Hern R, et al. Non-pharmacological intervention for breathlessness in lung cancer. Palliat Med 1996;10:299-305.

102 Filshie J, Penn K, Ashley S, et al. Acupuncture for the relief of cancer-related breathlessness. Palliat Med 1996;10:145-50.
103 Galbraith S, Fagan P, Perkins P, et al. Does the use of a handheld fan improve chronic dyspnea? A randomized, controlled, crossover trial. J Pain Symptom Manage 2010;39:831-8.

104 Jennings AL, Davies AN, Higgins JP, et al. Opioids for the palliation of breathlessness in terminal illness. Cochrane Database Syst Rev 2001;(4):CD002066.

105 Booth $\mathrm{S}$, Anderson $\mathrm{H}$, Swannick $\mathrm{M}$, et al. The use of oxygen in the palliation of breathlessness. A report of the expert working group of the Scientific Committee of the Association of Palliative Medicine. Respir Med 2004;98:66-77.

106 Philip J, Gold M, Milner A, et al. A randomized, double-blind, crossover trial of the effect of oxygen on dyspnea in patients with advanced cancer. J Pain Symptom Manage 2006;32:541-50.

107 Abernethy AP, McDonald CF, Frith PA, et al. Effect of palliative oxygen versus room air in relief of breathlessness in patients with refractory dyspnoea: a double-blind, randomised controlled trial. Lancet 2010;376:784-93.

108 Uronis HE, Currow DC, McCrory DC, et al. Oxygen for relief of dyspnoea in mildlyor non-hypoxaemic patients with cancer: a systematic review and meta-analysis. Br J Cancer 2008:98:294-9.

109 Clemens KE, Quednau I, Klaschik E. Use of oxygen and opioids in the palliation of dyspnoea in hypoxic and non-hypoxic palliative care patients: a prospective study. Support Care Cancer 2009;17:367-77.

110 Currow DC, Agar M, Smith J, et al. Does palliative home oxygen improve dyspnoea? A consecutive cohort study. Palliat Med 2009;23:309-16.

111 Quantrill SJ, White R, Crawford A, et al. Short burst oxygen therapy after activities of daily living in the home in chronic obstructive pulmonary disease. Thorax 2007;62:702-5.

112 Nandi K, Smith AA, Crawford A, et al. Oxygen supplementation before or after submaximal exercise in patients with chronic obstructive pulmonary disease. Thorax 2003:58:670-3.

113 Lewis CA, Eaton TE, Young $P$, et al. Short-burst oxygen immediately before and after exercise is ineffective in nonhypoxic COPD patients. Eur Respir $J$ 2003;22:584-8

114 O'Driscoll BR, Neill J, Pulakal S, et al. A crossover study of short burst oxygen therapy (SBOT) for the relief of exercise-induced breathlessness in severe COPD. BMC Pulm Med 2011;11:23.

115 Evans TW, Waterhouse JC, Carter A, et al. Short burst oxygen treatment for breathlessness in chronic obstructive airways disease. Thorax 1986;41:611-15.

116 Stevenson NJ, Calverley PM. Effect of oxygen on recovery from maximal exercise in patients with chronic obstructive pulmonary disease. Thorax 2004:59:668-72.

117 Eaton T, Fergusson W, Kolbe J, et al. Short-burst oxygen therapy for COPD patients: a 6-month randomised, controlled study. Eur Respir J 2006;27:697-704.

118 Cohen AS, Burns B, Goadsby PJ. High-flow oxygen for treatment of cluster headache: a randomized trial. JAMA 2009;302:2451-7.

119 Kudrow L. Response of cluster headache attacks to oxygen inhalation. Headache 1981;21:1-4.

120 Fogan L. Treatment of cluster headache. A double-blind comparison of oxygen $\mathrm{v}$ air inhalation. Arch Neurol 1985;42:362-3.

121 Rozen TD. High oxygen flow rates for cluster headache. Neurology 2004:63:593.

122 Backx APM, Haane DYP, De Ceuster L, et al. Cluster headache and oxygen: is it possible to predict which patients will be relieved? A retrospective cross-sectional correlation study. J Neurol 2010;257:1533-42.

123 NICE. NICE Guideline for the diagnosis and management of headaches in young people and adults. NICE, 2012.

124 Johns DP, Rochford PD, Streeton JA. Evaluation of six oxygen concentrators Thorax 1985:40:806-10.

125 Hall LW, Kellagher RE, Fleet KJ. A portable oxygen generator. Anaesthesia 1986:41:516-18.

126 Gould GA, Scott W, Hayhurst MD, et al. Technical and clinical assessment of oxygen concentrators. Thorax 1985;40:811-16.

127 [No authors listed]. Home controls of a sample of 2,414 oxygen concentrators Sous-Commission Technique ANTADIR. Eur Respir J 1991;4:227-31.

128 Burioka N, Takano K, Hoshino E, et al. Clinical utility of a newly developed pressure swing adsorption-type oxygen concentrator with a membrane humidifier. Respiration 1997:64:268-72.

129 Burioka N, Takano K, Suyama H, et al. Efficacy of newly developed pressure swing adsorption type oxygen concentrator with membrane humidifier: comparison with conventional oxygen concentrator with bubble water humidifier. Intern Med 1997:36:861-4.

130 Pesce LI, Bassi GN, Santovito A. Clinical usefulness of a new portable oxygen concentrator. Monaldi Arch Chest Dis 1994:49:444-6.

131 Shiner RJ, Zaretsky U, Mirali M, et al. Evaluation of domiciliary long-term oxygen therapy with oxygen concentrators. Isr J Med Sci 1997;33:23-9.

132 Jackson M, Shneerson J. An evaluation of the use of concentrators for domiciliary oxygen supply for less than $8 \mathrm{~h}$ day-1. Respir Med 1998;92:250-5.

133 Cuvelier A, Nuir JF, Chakroun N, et al. Refillable oxygen cylinders may be an alternative for ambulatory oxygen therapy in COPD. Chest 2002;122:451-6.

134 Strickland SL, Hogan TM, Hogan RG, et al. A randomized multi-arm repeated-measures prospective study of several modalities of portable oxygen 
delivery during assessment of functional exercise capacity. Respir Care 2009:54:344-9.

135 Lock SH, Blower G, Prynne M, et al. Comparison of liquid and gaseous oxygen for domiciliary portable use. Thorax 1992;47:98-100.

136 Nasilowski J, Przybylowski T, Zielinski J, et al. Comparing supplementary oxygen benefits from a portable oxygen concentrator and a liquid oxygen portable device during a walk test in COPD patients on long-term oxygen therapy. Respir Med 2008:102:1021-5.

137 Andersson A, Ström K, Brodin H, et al. Domiciliary liquid oxygen versus concentrator treatment in chronic hypoxaemia: a cost-utility analysis. Eur Respir J 1998;12:1284-9.

138 Katsenos S, Charisis A, Daskalopoulos G, et al. Long-term oxygen therapy in chronic obstructive pulmonary disease: the use of concentrators and liquid oxygen systems in north-western Greece. Respiration 2006;73:777-82.

139 Czajkowska-Malinowska M, Poltyn B, Ciesielska A, et al. Comparison of the results of long term oxygen therapy in patients treated sequentially using stationary or a portable source of oxygen. Pneumonol Alergol Pol 2012;80:308-16.

140 Okubadejo AA, Paul EA, Wedzicha JA. Domiciliary oxygen cylinders: indications, prescription and usage. Respir Med 1994;88:777-85.

141 Bazuaye EA, Stone TN, Corris PA, et al. Variability of inspired oxygen concentration with nasal cannulas. Thorax 1992;47:609-11.

142 Paul J, Otvos T. Comparison of nasal cannulas and the OxyArm in patients requiring chronic domiciliary oxygen therapy. Can Respir J 2006;13:421-6.

143 Domingo C, Roig J, Coll R, et al. Evaluation of the use of three different devices for nocturnal oxygen therapy in COPD patients. Respiration 1996;63:230-5.
144 Moore-Gillon JC, George RJ, Geddes DM. An oxygen conserving nasal cannula. Thorax 1985:40:817-19.

145 Roberts CM, Bell J, Wedzicha JA. Comparison of the efficacy of a demand oxygen delivery system with continuous low flow oxygen in subjects with stable COPD and severe oxygen desaturation on walking. Thorax 1996;51:831-4.

146 Braun SR, Spratt G, Scott GC, et al. Comparison of six oxygen delivery systems for COPD patients at rest and during exercise. Chest 1992;102:694-8.

147 Marti S, Pajares V, Morante F, et al. Are oxygen-conserving devices effective for correcting exercise hypoxemia? Respir Care 2013;58:1606-13.

148 Chatburn RL, Lewarski JS, McCoy RW. Nocturnal oxygenation using a pulsed-dose oxygen-conserving device compared to continuous flow. Respir Care 2006:51:252-6.

149 Leggett RJ, Flenley DC. Portable oxygen and exercise tolerance in patients with chronic hypoxic cor pulmonale. BMJ 1977;2:84-6.

150 Crisafulli E, Costi S, De BF, et al. Effects of a walking aid in COPD patients receiving oxygen therapy. Chest 2007;131:1068-74.

151 Centers for Disease Control and Prevention. Fatal fires associated with smoking during long-term oxygen therapy —Maine, Massachusetts, New Hampshire, and Oklahoma, 2000-2007. MMWR Morb Mortal Wkly Rep 2008;57:852-4.

152 Robb BW, Hungness ES, Hershko DD, et al. Home oxygen therapy: adjunct or risk factor? Burn Care Rehabil 2003;24:403-6; discussion 02.

153 Chang TT, Lipinski CA, Sherman HF. A hazard of home oxygen therapy. Burn Care Rehabil 2001;22:71-4; discussion 70-1.

154 Amani H, Lozano DD, Blome-Eberwein S. Brother, have you got a light? Assessing the need for intubation in patients sustaining burn injury secondary to home oxygen therapy. J Burn Care Res 2012;33:e280-5. 


\section{APPENDIX 1: PROTOCOL FOR AMBULATORY OXYGEN THERAPY ASSESSMENT}

\section{AIMS OF ASSESSMENT FOR EACH PATIENT}

(1) To determine if there is desaturation on exercise, defined as a drop in SpO2 of $\geq 4 \%$ to $<90 \%$

(2) To determine the most appropriate device and setting to correct exercise desaturation

\section{CONSIDERATIONS}

- This protocol is designed to be generic and can be adapted for any valid and repeatable walking test.

- A 6 Minute Walking Test (6MWT) should be performed over a 30m course (cones $29 \mathrm{~m}$ apart), but it is recognised that due to a lack of space a modified 10m-6MWT (cones $9 \mathrm{~m}$ apart) may be used as an alternative $(1,2)$. Incremental and Endurance Shuttle Walking Tests (ESWT) are performed over a $10 \mathrm{~m}$ course (cones $9 \mathrm{~m}$ apart). There is some evidence to show that endurance tests, such as the ESWT, may be more sensitive than standard tests $(3,4)$.

- Desaturation during baseline endurance shuttle walking test (ESWT) has been found to predict required flow rate (see annex 1). This is unlikely to predict as robustly when desaturations produced during other walking tests are used but may give some guidance.

- A practice walk test should be performed and without one the improvement in walking distance from air to oxygen is likely to be overestimated.

- Local policy and individual patient capabilities will affect the maximum number of tests performed in one appointment. Two appointments may be required to titrate oxygen fully.

- It is not possible to correct $\mathrm{SpO}_{2}$ in every patient to $>90 \%$ using 6 litres per minute (lpm) oxygen or the maximum settings on other devices. In this situation discussion with patient and their consultant may help determine if a higher flow rate may be suitable. Portability and / or duration of use declines considerably above $6 \mathrm{lpm}$.

- Authors have described a dose response to oxygen i.e. for each increase in flow rate there is an increase in exercise performance (3). Those whose performance has not improved on oxygen should therefore be trialled on a higher flowrate / setting.

- Carrying the cylinder / device negates the effect of the oxygen but wheeling it does not (5). Therefore patients must have AOT flow rate / setting titrated while carrying / wheeling the oxygen device as they plan to use it in everyday life.

- Different oxygen devices weigh different amounts and oxygen conservers vary in sensitivity and functionality which result in devices responding differently to different patients (6). Patients must have the flow / setting titrated on the device that they are to be prescribed.

\section{EQUIPMENT REQUIRED}

- Long, flat, corridor at least $32 \mathrm{~m}$ long (12m for modified $6 \mathrm{MWT}$ )

- 2 cones

- 2 chairs (placed beyond each cone)

- Stopwatch/CD \& CD player

- Ambulatory oxygen equipment (hired/supplied by oxygen provider)

- Nasal cannulae

- Pulse oximeter

- Modified BORG breathlessness scale

- Oxygen risk assessment

- Patient information leaflets

\section{PREPARATION}

- Explain the purpose of AO

- Outline the AO assessment process

- Gain informed consent for assessment

- Confirm indication for AO (including outdoor mobility)

- Complete risk assessment

- Ensure 20 mins rest before walking test (included in discussion time)

- Set up walking test circuit

- Read / play test instructions

- Ask if the patient has any questions

- Perform practice test

- Ensure further 20 mins rest before retest

\section{DEMONSTRATING A POSITIVE IMPROVEMENT WITH AO}

2 out of 3 of the markers below are required to show that the patient benefits from AO.

- $\mathrm{SpO} 2 \mathrm{~s} \geq 90 \%$ throughout

- $\geq 10 \%$ increase in walking distance from baseline (7)

- Improvement in BORG of at least 1 point from baseline (8)

\section{FOLLOW-UP}

\section{8 week review:}

Check patient's concordance with the oxygen order (call the oxygen delivery company to determine their usage) and compare this with the patient's diary card when they attend. Discuss any discrepancies or issues highlighted.

Troubleshoot any device issues. Review device and oxygen order as required.

Annual review:

Reassess using current prescription and adjust flow rate and device as required. 
Carry out baseline walk test on air

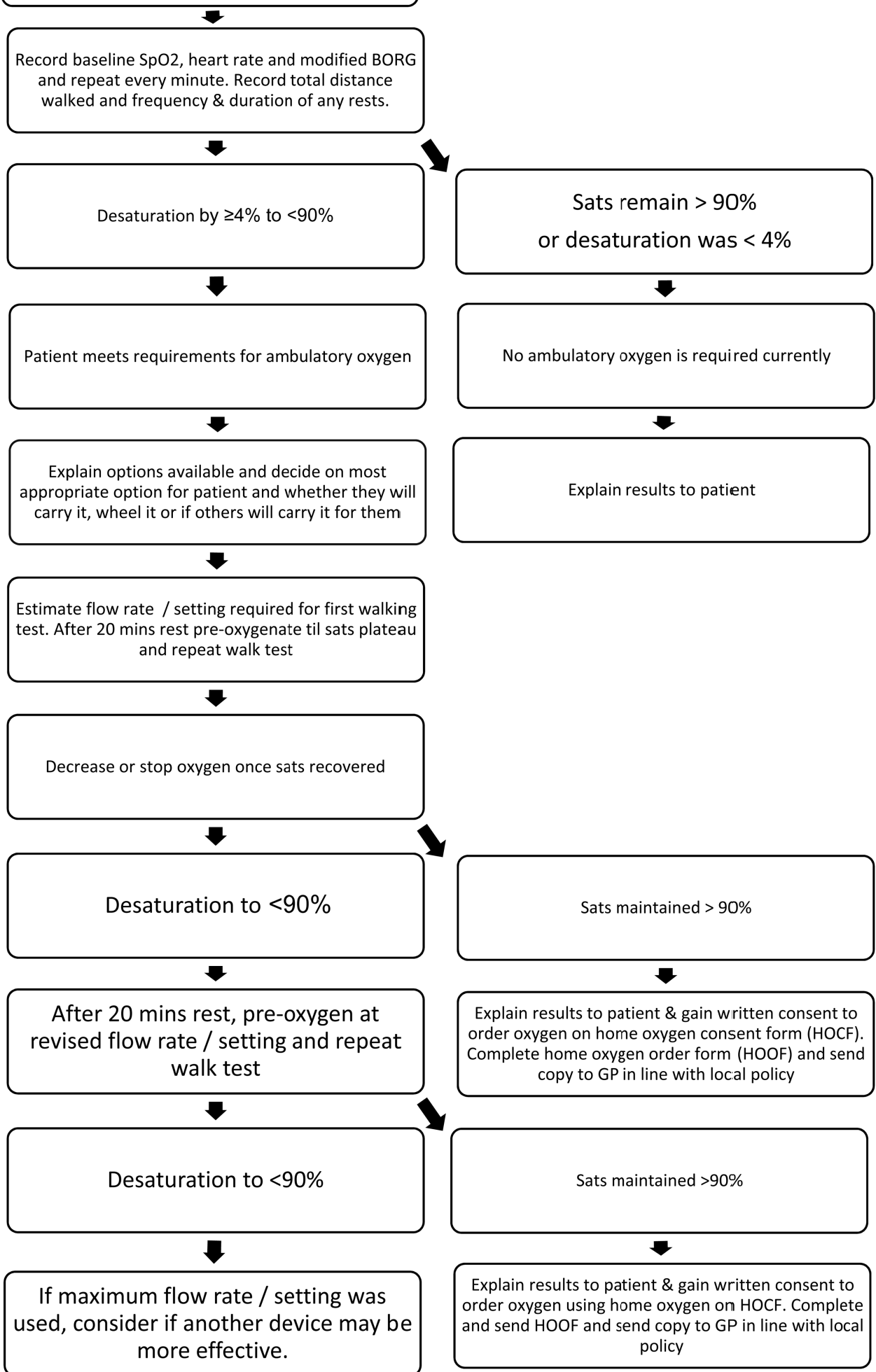




\section{ANNEX 1}

Suggested AO flow rates according to baseline ESWT desaturations (9):

\begin{tabular}{ll}
\hline Oxygen saturation range (\%) & Suggested AO flow rate (I/min) \\
\hline $86-89$ & 3 \\
$80-85$ & 4 \\
$74-79$ & 5 \\
73 or below & 6 \\
\hline
\end{tabular}

\section{References}

(1) Beekman E, Mesters I, Gosselink R, Klaassen MPM, Hendriks EJM, Van Schayck OCP, De Bie RA. The first reference equations for the 6-minute walking distance over a 10 metre course. Thorax 2014. http://thorax.bmj.com/content/early/2014/04/23/ thoraxjnl-2014-205228

(2) Crapo RO, Casaburi R, Coates AL, et al (2002) American Thoracic Society Statement - Guidelines for the six-minute walk test. American Journal of Respiratory and Critical Care Medicine. 166 p 111-117. http:/ajrccm.atsjournals.org/cgi/content/full/166/1/ 111 [accessed 02/07/2007]

(3) Davidson AC, Leach R, George RJD, Geddes DM. 1988. Supplemental oxygen and exercise ability in chronic obstructive airways disease. Thorax, 43: 965-971.

(4) Ambulatory oxygen therapy assessment: a comparative study of incremental shuttle and 6-minute walking tests A. Lewko, J. Marshall, R. Garrod Physiotherapy - December 2007 (Vol. 93, Issue 4, Pages 261-266, DOI: 10.1016/j. physio.2007.03.002).

(5) Leggett RJE, Flenley DC. 1977. Portable oxygen and exercise tolerance in patients with chronic hypoxic cor pulmonale. British Medical Journal, ii: 84-86.

(6) Bliss PL, McCoy RW, Adams AB. 1999. A bench Study Comparison of Demand Oxygen Delivery Systems and Continuous Flow Oxygen. Respiratory Care, 44 (8): 925-931.

(7) Dyer F, Callaghan J, Cheema K, Bott J. Ambulatory oxygen improves the effectiveness of pulmonary rehabilitation in selected patients with chronic obstructive pulmonary disease. Chronic Respiratory Disease 2012;9:83-91

(8) Ries AL. Minimally Clinically Important Difference for the UCSD Shortness of Breath Questionnaire, Borg Scale, and Visual Analog Scale. Journal of Chronic Obstructive Pulmonary Disease 2005; 2 (1) 105-110.

(9) Cornish L, Dyer F, Cheema K, Bott J. Is it possible to predict ambulatory oxygen (AO) requirements? Thorax 2013;68:Suppl 3 A92 doi:10.1136/thoraxjnl-2013-204457.188 


\section{BTS Guidelines}

APPENDIX 2: ASSESSMENT REFERRAL FORM

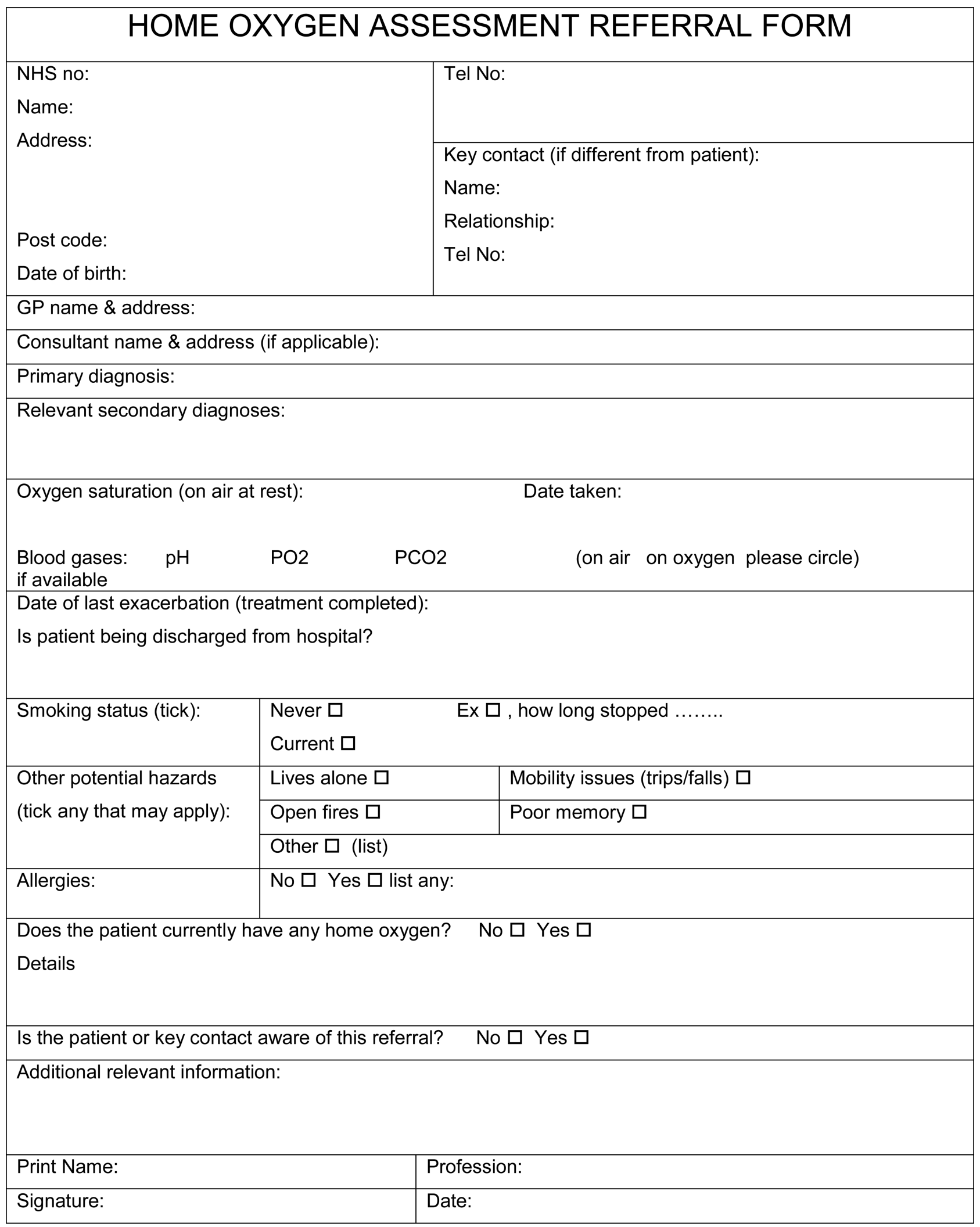

Please return to Home Oxygen Assessment Service fax:

For urgent referral tel: 


\section{APPENDIX 3: ASSESSMENT PROTOCOL FOR PALLIATIVE OXYGEN}

There is no consensus for the correct clinical assessment strategy for the use of oxygen in palliative care, although multiple tools exist for assessing dyspnoea. This assessment protocol is suggested best practice by the guideline group and applies to patients with cancer or end stage cardio-respiratory disease who are experiencing intractable breathlessness, who are hypoxaemic with resting $\mathrm{SpO}_{2}<92 \%$ or who are normoxaemic but in whom all other approaches have been exhausted. The Numerical Rating Scale score is recommended as this approach was used in evidence sited. First ensure patient is on maximum treatment for underlying diseases where possible and reversible causes for breathlessness have been or are being treated optimally.

- As distress from breathlessness can be multi-dimensional, ensure psycho-social factors have been assessed and addressed.

- Trial of non-pharmacological measures including teaching of breathing relaxation and life modifying strategies by involving physio and occupational therapists.

- Trial of hand held fan before consideration of oxygen therapy.

- Assess response to opioids if they have been tried.

- Check $\mathrm{SpO}_{2}$ using pulse oximetry at rest and/ or after exertion.

The subjective severity and intensity of breathlessness should therefore be recorded regularly to evaluate the degree of suffering caused and the effect of treatment. A numerical rating scale (NRS) from 0 to 10 has been found useful for this purpose $(0=$ no shortness of breath, $10=$ worst shortness of breath imaginable). Treatment should focus on patients with dyspnoea scores (NRS) of $\geq 4$, and especially those with scores $\geq 7$. Recurrent assessment with standardized scales is prudent, especially when using an $\mathrm{N}$-of- 1 approach, as it is difficult to predict which patients will benefit (1).

\section{PRESCRIPTION}

As distress from breathlessness is not correlated to degree of hypoxemia, the flow rates for symptom relief in the studies identified range from 2-5 litres/min. It is suggested therefore that oxygen flow rates be determined by symptom score on an individual basis rather than $\mathrm{SpO}_{2}$ reading. Additional consideration needs to be given to potential risks of hypercapnia if oxygen is given at higher flow rates.

\section{EQUIPMENT}

Concentrator or cylinder as determined by patient's needs.

\section{FOLLOW UP}

Oxygen therapy like any pharmacological intervention should be best considered on trial basis and be reviewed regularly while balancing between benefits and risks.

Most benefit is likely to occur in the first 24 hours, and nearly all symptomatic and functional improvements within the first 3 days of use (1). Follow-up and assessment of response should fit with these timescales.

1. Nonoyama ML, Brooks D, Guyatt GH, et al. Effect of oxygen on health quality of life in patients with chronic obstructive pulmonary disease with transient exertional hypoxemia. Am J Respir Crit Care Med 2007;176(4):343-9 


\section{BTS Guidelines}

\section{APPENDIX 4: RISK ASSESSMENT TOOLS*}

*The risk assessment templates provided have not been validated.

\section{FIELD BASED RISK ASSESSMENT REPORT TEMPLATE AS USED BY HOME OXYEN PROVIDER COMPANY}

Written confirmation that the risk assessment has been conducted at the Patient's home at the due date and report of the findings of the assessment shall include, but not necessarily be limited to, the following information:-

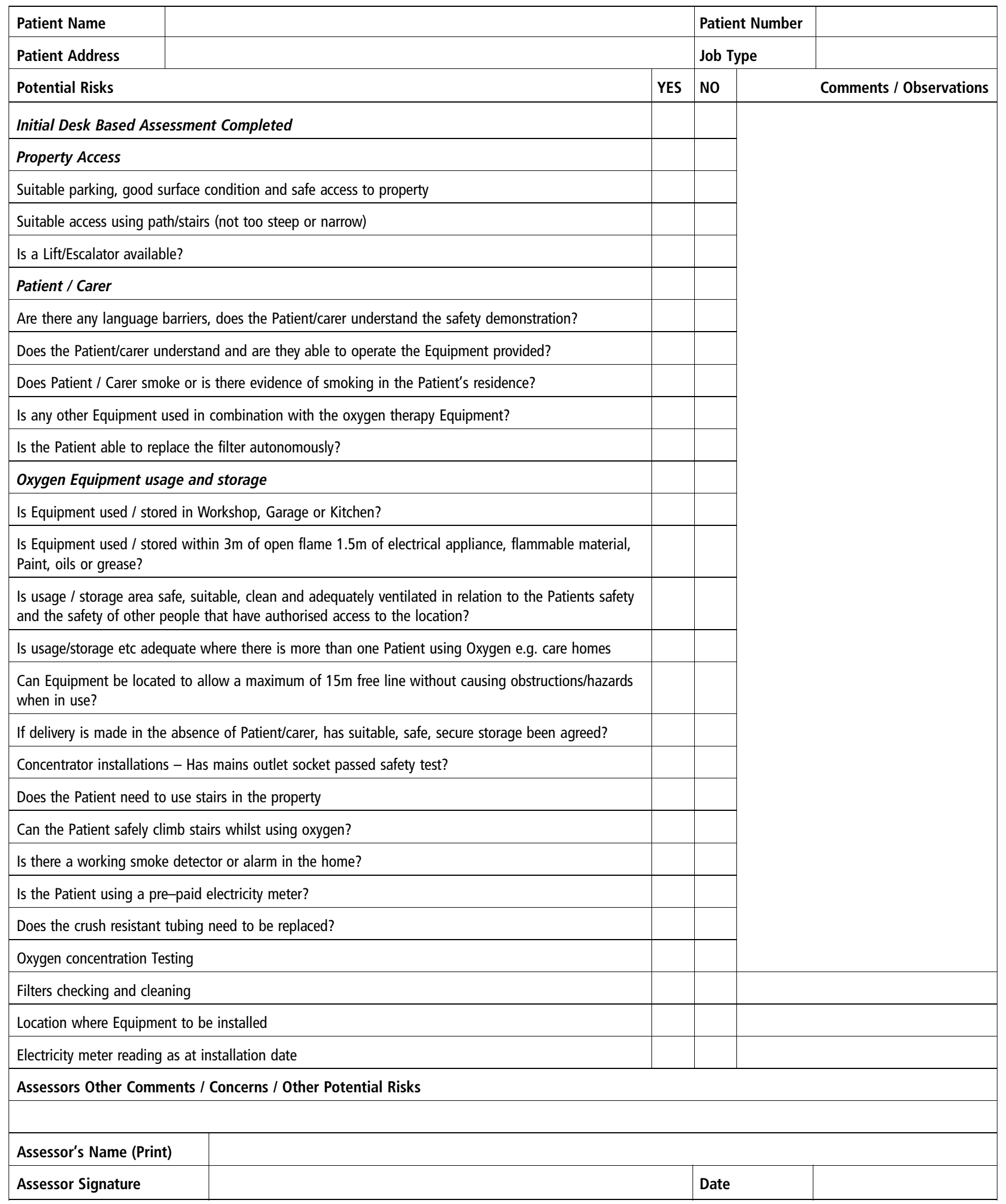


$\underline{\text { Risk Assessment Form (Wirral NHS Home Oxygen assessment service)* }}$

Location/Activity:

Oxygen Therapy

Assessment date:

Patient's Full Name: $\quad$ Date of birth: $\quad$ NHS N ${ }^{\circ}$

Assessor:

Signature:

Review date:

Risk Description/Source

IF THE PATIENT HAS AN AIR FLOW PRESSURE RELIEVING MATTRESS YOU MUST DISCUSS THE POTENTIAL RISKS AND DOCUMENT IT

\begin{tabular}{|c|c|c|c|c|c|c|}
\hline Ref & Hazards & Risks & People at risk & Current Control Measures & $L \times C=R$ & $\begin{array}{l}\text { Is further action } \\
\text { required }(\mathrm{Y} / \mathrm{N})\end{array}$ \\
\hline 1. & Smoking & $\begin{array}{l}\text { Fire } \\
\text { Facial burns }\end{array}$ & $\begin{array}{l}\text { Community Nursing Staff } \\
\text { Patients and carers } \\
\text { Patients }\end{array}$ & $\begin{array}{l}\text { (1) Instruct patients, carers and visitors not to smoke in any } \\
\text { part of the house where oxygen is used } \\
\text { (2) Patient/carer to sign "smoking and oxygen" advice form } \\
\text { (3) Arrange for removal of any oxygen equipment not in } \\
\text { regular use } \\
\text { (4) Fire breaks never to be removed from tubing supplied by } \\
\text { oxygen provider } \\
\text { (5) Ensure smoke detectors are fitted and in working order }\end{array}$ & & \\
\hline 2. & $\begin{array}{l}\text { Exposure to naked flames from open/ } \\
\text { gas fires/candles and cooking } \\
\text { appliances. }\end{array}$ & Explosion and fire & $\begin{array}{l}\text { Community Nursing Staff } \\
\text { Patients and carers }\end{array}$ & $\begin{array}{l}\text { (1) Advise patient to maintain a safe distance from fires } \\
\text { and naked flame appliances as instructed by oxygen } \\
\text { provider } \\
\text { (2) Oxygen must be positioned and stored as directed by } \\
\text { oxygen provider }\end{array}$ & & \\
\hline 3. & $\begin{array}{l}\text { Kinking or entrapment of tubing in/ } \\
\text { under furniture, doors, wheels }\end{array}$ & Restriction of or no Oxygen supply & Patient & $\begin{array}{l}\text { (1) Check there are no kinks in the tubing } \\
\text { (2) Check that the tubing is not trapped between furniture } \\
\text { or trapped e.g. under bed wheels } \\
\text { (3) Only tubing supplied by the oxygen provider is to be } \\
\text { used on cylinders and concentrators } \\
\text { (4) Encourage piped oxygen if there is excessive tubing }\end{array}$ & & \\
\hline 4. & Alcohol hand rubs/gels & Combustion & $\begin{array}{l}\text { Community nursing staff } \\
\text { Patients and carers }\end{array}$ & $\begin{array}{l}\text { (1) Ensure hands are adequately dried after the use of } \\
\text { alcohol gels. }\end{array}$ & & \\
\hline 5 & Use of oil based emollients & Local burning of affected area & Patient & $\begin{array}{l}\text { (1) Instruct patients (or carers) not to use oil based } \\
\text { emollients on patients nostrils }\end{array}$ & & \\
\hline 6. & $\begin{array}{l}\text { Patient/Carers not aware on how to } \\
\text { obtain replacement cylinders }\end{array}$ & Running out of oxygen & Patient & $\begin{array}{l}\text { (1) Ensure patient has information leaflet from company } \\
\text { supplying oxygen } \\
\text { (2) Check patient/carer has contact details on how to } \\
\text { obtain/replace oxygen cylinder. }\end{array}$ & & \\
\hline
\end{tabular}




\begin{tabular}{|c|c|c|c|c|c|c|}
\hline Ref & Hazards & Risks & People at risk & Current Control Measures & $L \times C=R$ & $\begin{array}{l}\text { Is further action } \\
\text { required }(\mathrm{Y} / \mathrm{N})\end{array}$ \\
\hline 7. & Tubing & Trips and falls & $\begin{array}{l}\text { Community Nursing Staff } \\
\text { Patients and carers }\end{array}$ & $\begin{array}{l}\text { (1) Advise patients and carers to check position of tubing } \\
\text { daily to minimise risks of falls } \\
\text { (2) Advise patients and carers to check position of tubing, } \\
\text { particularly if patient using a walking frame etc } \\
\text { (3) Current oxygen tubing must be of an appropriate length } \\
\text { to meet the needs of the patient. }\end{array}$ & & \\
\hline 8. & Power supply cut off to concentrator & No oxygen supply & Patient & $\begin{array}{l}\text { (1) Check patient has a back up cylinder } \\
\text { (2) Educate patient not to use back up cylinder unless there } \\
\text { is power failure to concentrator }\end{array}$ & & \\
\hline 9 & $\begin{array}{l}\text { Unauthorised adjustment of flow rate } \\
\text { on oxygen equipment }\end{array}$ & $\begin{array}{l}\text { Worsening respiratory failure in oxygen sensitive } \\
\text { patients }\end{array}$ & Patient & $\begin{array}{l}\text { (1) Educate patient on the reason for oxygen } \\
\text { (2) Inform patient/carer of the prescribed flow rate and } \\
\text { hours of use } \\
\text { (3) Ensure patient/carer understands how to operate } \\
\text { equipment safely } \\
\text { (4) Inform patient/carer on the importance of not adjusting } \\
\text { oxygen flow rate without seeking appropriate clinical } \\
\text { advice and assessment } \\
\text { (5) Very oxygen sensitive patients will be issued with an } \\
\text { alert card and appropriate oxygen mask and tubing for } \\
\text { use in ambulance transfers. }\end{array}$ & & \\
\hline 10 & $\begin{array}{l}\text { Non compliance with assessment and/ } \\
\text { or review process }\end{array}$ & $\begin{array}{l}\text { Risks will not be identified or managed. Oxygen } \\
\text { prescription may not be appropriate for the } \\
\text { patient's clinical need. }\end{array}$ & Patient & $\begin{array}{l}\text { (1) All except terminally ill patients should be formally } \\
\text { assessed prior to commencing oxygen therapy. } \\
\text { (2) Patients will be recalled for review according to national } \\
\text { guidance } \\
\text { (3) Assessment and review will be undertaken at a mutually } \\
\text { convenient time and place. }\end{array}$ & & \\
\hline 11 & $\begin{array}{l}\text { Non compliance with oxygen } \\
\text { prescription }\end{array}$ & Hypoxia remains untreated & Patient & $\begin{array}{l}\text { (1) Patients will be educated on when and how to use } \\
\text { oxygen at the time of prescribing. } \\
\text { (2) Reason for oxygen will be discussed at each review. } \\
\text { (3) Significant carers, family and other HP's involved with } \\
\text { the patient to be educated on why oxygen has been } \\
\text { prescribed. }\end{array}$ & & \\
\hline
\end{tabular}

$\square$ Green Low Risk (04)

$\square$ Yellow/Amber Moderate Risk (5-12)

$\square$ Red High to extreme Risk (15-25)
Managed by COPD and oxygen service $=$ Acceptable risk. Routine review

Managed by Senior Managers $=$ Action required

Managed by Director Level=Serious risk requiring immediate action. 
RISK SCORING MATRIX

\begin{tabular}{|c|c|c|c|c|c|}
\hline \multirow[b]{2}{*}{ Likelihood } & \multicolumn{5}{|l|}{ Consequence } \\
\hline & Insignificant 1 & Minor 2 & Moderate 3 & Major 4 & Catastrophic 5 \\
\hline Certain 5 & 5 & 10 & 15 & 20 & 25 \\
\hline Likely 4 & 4 & 8 & 12 & 16 & 20 \\
\hline Possible 3 & 3 & 6 & 9 & 12 & 15 \\
\hline Unlikely 2 & 2 & 4 & 6 & 8 & 10 \\
\hline Rare 1 & 1 & 2 & 3 & 4 & 5 \\
\hline
\end{tabular}

PRIORITY AND ACTION

\begin{tabular}{|c|c|c|c|c|}
\hline $\begin{array}{l}\text { Risk } \\
\text { Colour }\end{array}$ & $\begin{array}{l}\text { Risk } \\
\text { rating }\end{array}$ & $\begin{array}{l}\text { Risk } \\
\text { level }\end{array}$ & Identifier & Action required \\
\hline GREEN & $0-4$ & Low & $\begin{array}{l}\text { Control measures in place or risk of harm is } \\
\text { insignificant }\end{array}$ & Long term action with routine review \\
\hline AMBER & $5-12$ & Medium & $\begin{array}{l}\text { Likelihood of major harm if control measures not } \\
\text { implemented }\end{array}$ & Action is needed in the medium term \\
\hline RED & $15-25$ & High & Significant probability of major harm & $\begin{array}{l}\text { Urgent action needed. Escalate to line manager, patient's GP and } \\
\text { senior manager. }\end{array}$ \\
\hline
\end{tabular}

\section{ESCALATION PATHWAY}

Low risk - Green (0-4)

Continue usual control measures, reinforce education, update documentation, see at next planned review.

Incident form to be completed if the patient sustains injury or harm related to oxygen. Copy for WUTH and PCT

\section{Medium risk - Yellow (5-12)}

Ensure all current safety control measures are in place including fire service involvement

Implement and reinforce control measures

Inform patient's GP and community nursing team/matron if appropriate.

Notify locality MDT for discussion and review

Involve carers/next of kin in discussion of safety issues

Review risk after 4 weeks

Incident form to be completed if the patient sustains injury or harm related to oxygen. Copy for WUTH and PCT

\section{High risk - Red (15-25)}

Ensure all current safety control measures are in place including fire service involvement

Urgent discussion with patient's GP

Urgent discussion with community nursing team/matron if appropriate

Inform next of kin, carers of the seriousness of the situation

Arrange for removal of oxygen and admission to hospital if removal of oxygen is likely to result in severe hypoxia

Incident form to be completed if oxygen removed or the patient sustains injury or harm related to oxygen. Copy for WUTH and PCT. 
Patient's Full Name: Date of birth: NHS No

Location/Activity: Assessment date:

Assessor: $\quad$ Signature: Review date:

\begin{tabular}{l|l|l|l|l}
\hline Ref & Further Action Implemented Short Term, Medium Term, Long Term & Responsible Person & Revised Risk rating L x C=R & $\begin{array}{l}\text { Are further assessments required if so list. } \\
\text { e.g. COSHH }\end{array}$ \\
\hline 1 & $\begin{array}{l}\text { Arrange 6monthly review. Review risk assessment annually. } \\
\text { Check safety implications at every visit. }\end{array}$ & & \\
\hline 2 & As above & & \\
3 & As above & & \\
4 & As above & & \\
5 & As above & & \\
6 & As above & & \\
7 & As above & & \\
8 & As above & & \\
9 & As above & & \\
10 & As above. Inform GP. & & \\
11 & As above. & & \\
\hline
\end{tabular}




\section{APPENDIX 5: HOME OXYGEN ORDER FORMS (HOOF) AND HOME OXYGEN CONSENT FORMS (HOCF) FORMS}

The Home Oxygen Order Form (HOOF) form for use in England and Wales comes in two parts:

Part A is used when the request is being made by non-specialist healthcare professionals and is usually for a temporary order pending review by a Home Oxygen Assessment service.

Part B should be used by Home Oxygen Assessment services once a patient has undergone formal assessment. It also allows for ordering of equipment in addition to the basic static concentrator and static cylinders.

Forms are available via the home oxygen supplier website relevant to each geographical area (or Part A HOOF is available from Primary Care Commissioning website.

http://www.pcc-cic.org.uk/article/home-oxygen-service-current-supply-contracts (accessed Jan 2015)

http://www.pcc-cic.org.uk/article/home-oxygen-order-form (accessed Jan 2015)

\section{APPENDIX 6: SAMPLE PATIENT INFORMATION LEAFLETS}

There are many examples of excellent patient information leaflets available from different Home Oxygen Assessment teams. The British Lung Foundation provides a wide range of information for patients with lung conditions: www.blf.org.uk

A new publication 'Oxygen treatment' will be available from April 2015: www.blf.org.uk/page/oxygen-treatment

The booklet will be available to order from the BLF shop: Patient: http://shop.blf.org.uk/products/oxygen-booklet

HCP: http://shop.blf.org.uk/products/oxygen-booklet-1 


\section{APPENDIX 7 : PRACTICAL POINTS FOR REMOVAL OF HOME OXYGEN}

Removal of home oxygen may be challenging and should be undertaken by experienced healthcare professionals. A multidisciplinary team approach including all healthcare professionals directly involved with the patient's care agreeing a plan may help the process. If this is not possible, communication of the removal plan to all relevant healthcare professionals is important.

Removal of home oxygen may not occur at one point but take time and multiple contacts. In order to remove home oxygen other interventions to manage breathlessness should be considered as an alternative where appropriate e.g. pulmonary rehabilitation, depression and anxiety management. The process may be stressful for the patient and their families or carers and should be handled sensitively.

It is helpful if the possibility of removal of home oxygen, and the circumstances in which this might occur, is raised with patients and their carers when home oxygen is first prescribed. This should be supported by written information.

\section{Reasons for removal of home oxygen therapy:}

1. Clinical

If the patient no longer meets the criteria for home oxygen on reassessment in a stable clinical state, then the removal process should be initiated. Consider the time and effort of removal in those patients with borderline arterial oxygen levels as they are likely to deteriorate in time, and a further assessment may be useful before initiating removal.

2. Patient adherence

Where patients do not use their prescribed oxygen for the period of time to gain clinical benefit (eg 15 hours per day with LTOT), they should be advised of the importance of this. An agreement should be reached with patients of an agreed period over which to improve adherence and if adherence is still suboptimal the oxygen should be removed.

Patients who do not use ambulatory oxygen therapy (AOT) despite leaving their house, or those who have AOT but do not leave their house, should again be similarly advised that this is a waste of NHS resources and an agreed period of time to improve adherence should be decided on, before reassessment. If there is no significant improvement in AOT use, then it should be removed.

3. Safety

If there are clear safety issues around the use of home oxygen a risk assessment should be undertaken (see Appendix 4). Serious incidents such as fires involving oxygen should lead to serious consideration of the removal of oxygen. Altering flow rates if not recommended by home oxygen team can result in harm and falls may result from piped oxygen tubing. There may be interventions that can minimise the risks and these should be initiated e.g. smoking cessation support in smokers; oxygen tubing being piped in and fixed rather than loose to reduce the risk of trips and falls; locking concentrators to avoid alteration in flow rates. Following initiation there should be a reassessment after a set agreed period.

Before removal of home oxygen you should be able to answer 'YES' to the following:

- Has the patient been reassessed by a health professional experienced in managing home oxygen or part of the home oxygen assessment team?

- Is there a clear indication for removal?

- Is the patient (and/or significant other) aware removal may occur?

- Have all interventions to improve adherence or reduce risk been considered and implemented with an evaluation following implementation?

- Have appropriate alternative treatment strategies been considered and implemented as part of the removal process?

- Have the wider health care team been part of the decision to remove home oxygen but if not informed of the decision prior to removal? 


\section{APPENDIX 8: GUIDELINE GROUP MEMBERS}

\section{Name}

Dr Maxine Hardinge, Chair Joe Annandale

Dr Simon Bourne

Dr Brendan Cooper

Lynn McDonnell

Angela Evans

Dr Daryl Freeman

Angela Green

Mr Colin Hawkey (dec)

Dr Sabrine Hippolyte

Vikki Knowles

Mrs Joan Ling

Professor William MacNee

Kathy Pye

Dr Jay Suntharalingam

Dr Vandana Vora

Dr Tom Wilkinson
Consultant Respiratory Physician Oxford University Hospitals NHS Trust

Respiratory Nurse Specialist, Hywel Dda University Health Board, Prince Philip Hospital, Llanelli Representing ARNS

Consultant Respiratory Physician Portsmouth NHS Trust Representing the Royal College of Physicians, London

Consultant Clinical Scientist, Lung Function and Sleep, Queen Elizabeth Hospital Birmingham Representing ARTP

Clinical Specialist Physiotherapist London Representing ACPRC

Specialist Practitioner, Staffordshire and Stoke-on-Trent Partnership Trust Representing ARTP

General Practitioner Representing PCRS-UK

Respiratory Physiotherapist Improvement Academy (Y\&H AHSN) Bradford Teaching Hospitals NHS FT

Patient/carer representative: 2011/2013

Respiratory Specialty Trainee London

Respiratory Nurse Specialist, Respiratory Care Team, Virgin Care, Surrey Representing PCRS -UK

Patient/carer representation: 2011/2012

Professor of Respiratory Medicine Edinburgh

Clinical Nurse Specialist Liverpool

Consultant Respiratory Physician Bath

Consultant Palliative Care Physician, Sheffield Representing Association for Palliative Medicine

Consultant Respiratory Physician Clinical and Experimental Sciences, Faculty of Medicine, University of Southampton, Southampton 